\title{
Plateau's Problem as a Singular Limit of Capillarity Problems
}

\author{
DARREN KING \\ The University of Texas at Austin \\ FRANCESCO MAGGI \\ The University of Texas at Austin \\ AND \\ SALVATORE STUVARD \\ The University of Texas at Austin
}

\begin{abstract}
Soap films at equilibrium are modeled, rather than as surfaces, as regions of small total volume through the introduction of a capillarity problem with a homotopic spanning condition. This point of view introduces a length scale in the classical Plateau's problem, which is in turn recovered in the vanishing volume limit. This approximation of area minimizing hypersurfaces leads to an energy based selection principle for Plateau's problem, points at physical features of soap films that are unaccessible by simply looking at minimal surfaces, and opens several challenging questions. (c) 2021 Wiley Periodicals LLC.
\end{abstract}

\section{Contents}

$0 . \quad$ Explanatory Note 2

1. Introduction 2

2. Cone, cup, and slab competitors, nucleation, and collapsing $\quad 13$

3. Existence of Generalized Minimizers: Proof of Theorem|1.4 34

4. The Euler-Lagrange Equation: Proof of Theorem $1.6 \quad 54$

5. Convergence to Plateau's Problem: Proof of Theorem|1.9 60

Appendix A. A Technical Fact on Sets of Finite Perimeter 67

Appendix B. Boundary Density Estimates

for the Harrison-Pugh Minimizers $\quad 69$

\begin{tabular}{lll}
\hline Appendix C. A Classical Variational Argument & 71
\end{tabular}

\begin{tabular}{ll}
\hline Bibliography & 72
\end{tabular}

Communications on Pure and Applied Mathematics, 0001-0075 (PREPRINT)

(C) 2021 Wiley Periodicals LLC. 


\section{Explanatory Note}

The present paper amends and replaces "Plateau's Problem as a Singular Limit of Capillarity Problems" by the same authors, which was published online in Comm. Pure Appl. Math. in 2020 with DOI 10.1002/cpa.21951; see [39]. The revision does not contain any major changes with respect to the previous version: in particular, all main results stated in [39] remain valid. The amendment is, nonetheless, necessary due to the presence of a technical error in the proof of [39, lemma 2.5] (construction of cup competitors). The present paper features a revised version of that lemma; see Lemma 2.5 below, with a correct proof. While the main changes only pertain to the actual construction of cup competitors, a few other minor changes were needed in the rest of the paper at places where cup competitors (and other competitors derived from them, such as exterior cup competitors and slab competitors) are used. This justifies the publication of a revised version of the entire paper in place of a corrigendum. For the interested reader, further comments on the technical complications in the construction of cup competitors that led to the mistake in [39] and the need for this revision can be found in Remark 2.6 and Figure 2.1.a, b).

\section{Introduction}

\subsection{Overview}

The theory of minimal surfaces with prescribed boundary data provides the basic model for soap films hanging from a wire frame: given an $(n-1)$-dimensional surface $\Gamma \subset \mathbb{R}^{n+1}$ without boundary, one seeks $n$-dimensional surfaces $M$ such that

$$
H_{M}=0, \quad \partial M=\Gamma,
$$

where $H_{M}$ is the mean curvature of $M$ (and $n=2$ in the physical case). A limitation of (1.1) as a physical model is that, in general, (1.1) may be nonuniquely solvable, including unstable (and thus, not related to observable soap films) solutions. Area minimization can be used to construct stable (and thus, physical) solutions, providing a strong motivation for the study of Plateau's problem; see [9]. Here we are concerned with a more elementary physical limitation of (1.1), namely, the absence of a length scale: if $M$ solves (1.1) for $\Gamma$, then $t M$ solves (1.1) for $t \Gamma$, no matter how large $t>0$ is.

Following [44], we introduce a length scale in the modeling of soap films by thinking of them as regions $E \subset \mathbb{R}^{n+1}$ with small volume $|E|=\varepsilon$. At equilibrium, the isotropic pressure at a point $y$ interior to the liquid but immediately close to its boundary $\partial E$ is

$$
p(y)=p_{0}+\sigma \vec{H}_{\partial E}(y) \cdot v_{E}(y)
$$

where $p_{0}$ is the atmospheric pressure, $\sigma$ is the surface tension, $v_{E}$ the outer unit normal to $E$, and $\vec{H}_{\partial E}$ the mean curvature vector of $\partial E$; at the same time, for any 

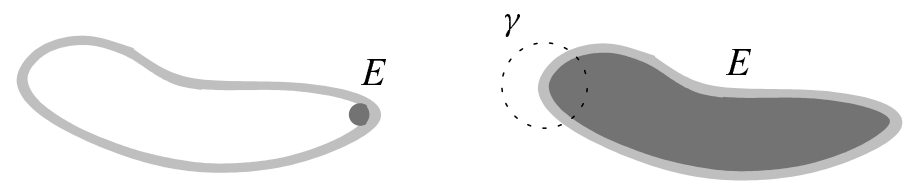

$I_{\delta}(\Gamma)$

FIGURE 1.1. Minimizers of the capillarity problem in the unusual container $\Omega$ consisting of the complement of a $\delta$-neighborhood $I_{\delta}(\Gamma)$ of a curve $\Gamma$ (depicted in light gray). The shape of $E$ is drastically different depending on whether or not a homotopic spanning condition is prescribed: (a) without a $\mathcal{C}$-spanning condition, we observe tiny droplets sitting near points of maximal mean curvature of $\partial \Omega$; (b) with a $\mathcal{C}$-spanning condition, small rounds droplets will not be admissible, and a different region of the energy landscape is explored; minimizers are now expected to stretch out and look like soap films.

two points $y, z$ inside the film we have

$$
p(y)-p(z)=\rho g(z-y) \cdot e_{n+1}
$$

where $\rho$ is the density of the fluid, $g$ is the gravity of Earth, and $e_{n+1}$ is the vertical direction. In the absence of gravity, (1.2) and (1.3) imply that $H_{E}=\vec{H}_{\partial E} \cdot v_{E}$ is constant along $\partial E$. A heuristic analysis shows that if $\partial E$ is representable locally by the two graphs $\left\{x \pm(h(x) / 2) v_{M}(x): x \in M\right\}$ defined by a positive function $h$ over an ideal midsurface $M$, then $H_{M}$ should be small, but nonzero (even in the absence of gravity); see [44, sec. 2]. As it is well-known, one cannot prescribe nonvanishing mean curvature with arbitrarily large boundary data; see, e.g., [21. 29]. Hence this point of view can potentially capture physical features of soap films that are not accessible by modeling them as minimal surfaces.

The goal of this paper is starting the analysis of the variational problem playing for (1.2) and (1.3) the role that Plateau's problem plays for (1.1). The new aspect is not in the energy minimized, but in the boundary conditions under which the minimization occurs. Indeed, the equivalence between the constancy of $H_{E}$ and the balance equations (1.2) and (1.3) leads us to work in the classical framework of Gauss' capillarity model for liquid droplets in a container. Given an open set $\Omega \subset \mathbb{R}^{n+1}$ (the container), the surface tension energy 11 of a droplet occupying the open region $E \subset \Omega$ is given by

$$
\sigma \mathcal{H}^{n}(\Omega \cap \partial E)
$$

where $\mathcal{H}^{n}$ denotes $n$-dimensional Hausdorff measure (surface area if $n=2$, length if $n=1$ ). In the case of soap films hanging from a wire frame $\Gamma$, we choose the

\footnotetext{
${ }^{1}$ For simplicity, we are setting to zero the adhesion coefficient with the container; see, e.g., $[26]$.
} 
(a)

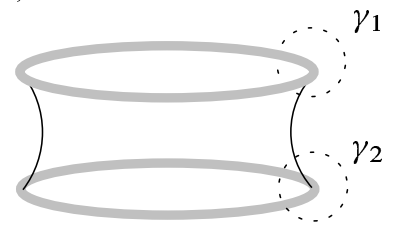

(b)

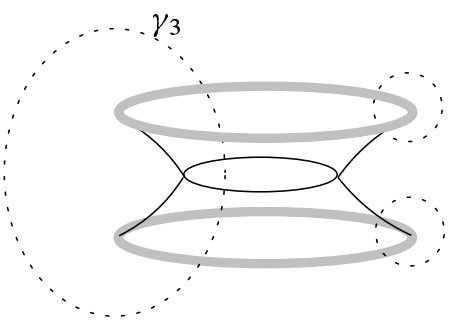

FiguRE 1.2. The variational problem (1.5) with $\Gamma$ given by two parallel circles centered on the same axis at a mutual distance smaller than their common radius. Different choices of $\mathcal{C}$ lead to different minimizers $S$ in $\ell:$ (a) if $\mathcal{C}$ is generated by the loops $\gamma_{1}$ and $\gamma_{2}$, then $S$ is the area minimizing catenoid; (b) if we add to $\mathcal{C}$ the homotopy class of $\gamma_{3}$, then $S$ is the singular area minimizing catenoid, consisting of two catenoidal necks, meeting at equal angles along a circle of $Y$-points bounding a "floating" disk. Such singular catenoid cannot be approximated in energy by smooth surfaces: hence the choice of casting $\ell$ in a class of nonsmooth surfaces.

complement of the "solid wire" $I_{\delta}(\Gamma)$ as our container $\Omega$; i.e., we set

$$
\Omega=\mathbb{R}^{n+1} \backslash I_{\delta}(\Gamma)
$$

where $I_{\delta}$ denotes the closed $\delta$-neighborhood of a set. The minimization of $\mathcal{H}^{n}(\Omega \cap$ $\partial E$ ) among open sets $E \subset \Omega$ with $|E|=\varepsilon$ leads indeed to finding minimizers whose boundaries have constant mean curvature. However, these boundaries will not resemble soap films at all, but will rather consist of small "droplets" sitting at points of maximal curvature for $I_{\delta}(\Gamma)$; see Figure 1.1] and [4, 22, 43] for more information.

To observe soap films rather than droplets, we must require that $\partial E$ stretches out to span $I_{\delta}(\Gamma)$. To this end, we exploit a beautiful idea introduced by Harrison and Pugh in [32] as slightly generalized in [13]. The idea is fixing a spanning class, i.e., a homotopically closed ${ }^{2}$ family $\mathcal{C}$ of smooth embeddings of $\mathbb{S}^{1}$ into $\Omega=\mathbb{R}^{n+1} \backslash I_{\delta}(\Gamma)$, and to say ${ }^{3}$ that a relatively closed set $S \subset \Omega$ is $\mathcal{C}$-spanning $I_{\delta}(\Gamma)$ if

$$
S \cap \gamma \neq \varnothing \quad \forall \gamma \in \mathcal{C} .
$$

Given a choice of $\mathcal{C}$, we have a corresponding version of Plateau's problem

(1.5) $\ell=\inf \left\{\mathcal{H}^{n}(S): S\right.$ is relatively closed in $\Omega$, and $S$ is $\mathcal{C}$-spanning $\left.I_{\delta}(\Gamma)\right\}$

as illustrated in Figure 1.2 . The variational problem $\psi(\varepsilon)$ studied here is thus a

\footnotetext{
${ }^{2}$ By this we mean that if $\gamma_{0}, \gamma_{1}$ are smooth embeddings of $\mathbb{S}^{1}$ into $\Omega, \gamma_{0} \in \mathcal{C}$, and there exists a continuous map $f:[0,1] \times \mathbb{S}^{1} \rightarrow \Omega$ with $f(t, \cdot)=\gamma_{t}$ for $t=0,1$, then $\gamma_{1} \in \mathcal{C}$.

${ }^{3}$ Notice that, in stating condition (1.4), the symbol $\gamma$ denotes the subset $\gamma\left(\mathbb{S}^{1}\right) \subset \Omega$. We are following here the same convention set in |13|.
} 
reformulation of $\ell$ as a capillarity problem with a homotopic spanning condition, namely:

$$
\psi(\varepsilon)=\inf \left\{\mathcal{H}^{n}(\Omega \cap \partial E): E \subset \Omega,|E|=\varepsilon, \Omega \cap \partial E \text { is } \mathcal{C}-\operatorname{spanning} I_{\delta}(\Gamma)\right\}
$$

for $\varepsilon>0$. We now give informal statements of our main results (e.g., we make no mention of singular sets or comment on reduced versus topological boundaries); see Section 1.2 for the formal ones.

\section{Existence of generalized minimizers and Euler-Lagrange equations (Theorem} 1.4 and Theorem 1.6)

There always exists a generalized minimizer $(K, E)$ for $\psi(\varepsilon)$ : that is, there exists a set $K \subset \Omega$, relatively closed in $\Omega$ and $\mathcal{C}$-spanning $I_{\delta}(\Gamma)$, and there exists an open set $E \subset \Omega$ with $\Omega \cap \partial E \subset K$ and $|E|=\varepsilon$, such that

$$
\psi(\varepsilon)=\mathcal{F}(K, E)=2 \mathcal{H}^{n}(K \backslash \partial E)+\mathcal{H}^{n}(\Omega \cap \partial E) .
$$

Moreover, $(K, E)$ minimizes $\mathcal{F}$ with respect to all its diffeomorphic images: in particular, $\Omega \cap \partial E$ has constant mean curvature $\lambda \in \mathbb{R}$ and $K \backslash \partial E$ has zero mean curvature.

\section{Convergence to the Plateau's problem (Theorem 1.9)}

We always have $\psi(\varepsilon) \rightarrow 2 \ell$ when $\varepsilon \rightarrow 0^{+}$, and if $\left(K_{j}, E_{j}\right)$ are generalized minimizers for $\psi\left(\varepsilon_{j}\right)$ with $\varepsilon_{j} \rightarrow 0^{+}$, then, up to extracting subsequences, we can find a minimizer $S$ for $\ell$ with

$$
2 \int_{K_{j} \backslash \partial E_{j}} \varphi+\int_{\partial E_{j}} \varphi \rightarrow 2 \int_{S} \varphi \quad \forall \varphi \in C_{c}^{0}(\Omega)
$$

as $j \rightarrow \infty$; in other words, generalized minimizers in $\psi\left(\varepsilon_{j}\right)$ with $\varepsilon_{j} \rightarrow 0^{+}$ converge as Radon measures to minimizers in the Harrison-Pugh formulation of Plateau's problem.

Example 1.1 (Volume and thickness in the noncollapsed case). Let $\Gamma$ consists of two points at distance $r$ in the plane, or of an $(n-1)$-sphere of radius $r$ in $\mathbb{R}^{n+1}$. For $\varepsilon$ small enough, $\psi(\varepsilon)$ should admit a unique generalized minimizer $(K, E)$, consisting of two almost flat spherical caps meeting orthogonally along the torus $I_{\delta}(\Gamma)$ (so that $K=\partial E$ and collapsing does not occur); see Figure 1.3(a). In general, we expect that when all the minimizers $S$ in $\ell$ are smooth, then generalized minimizers in $\psi(\varepsilon)$ are not collapsed, and, for small $\varepsilon, K=\partial E$ is a two-sided approximation of $S$, with $H_{E}=\psi^{\prime}(\varepsilon) \rightarrow 0$ and

$$
\psi(\varepsilon)=2 \ell+C \varepsilon^{2}+\mathrm{o}\left(\varepsilon^{2}\right) \quad \text { as } \varepsilon \rightarrow 0^{+}
$$

for a positive $C$. This insight is consistent with the idea (see [44]) that almost minimal surfaces arise in studying soap films with a thickness. In particular, volume and thickness will be directly related in terms of the geometry of $\Gamma$. Sending $\varepsilon \rightarrow 0^{+}$with $\Gamma$ fixed or, equivalently, considering $t \Gamma$ for large $t$ at $\varepsilon$ fixed, will 
(a)

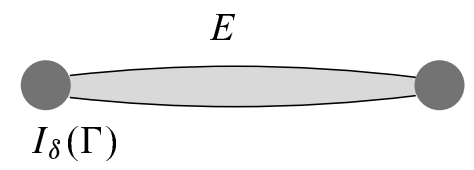

(b)

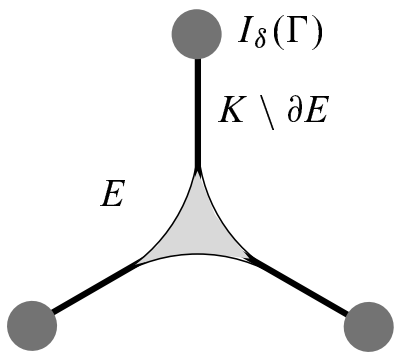

FIGURE 1.3. (a) If $\Gamma$ consists of two points, then the minimizer is not collapsed, and is bounded by two very flat circular arcs; (b) when $\Gamma$ consists of the vertexes of an equilateral triangle, the generalized minimizer is indeed collapsed. The three segments defining $K \backslash \partial E$ are depicted in bold, and $E$ is a negatively curved curvilinear triangle nested around the singular point of the unique minimizer of $\ell$.

make the thickness decrease until it reaches a threshold below which we do not expect soap films to be stable. A critical thickness can definitely be identified with the characteristic length scale of the molecules of surfactant, below which the model stops making sense. But depending on temperatures, actual soap films with even larger thicknesses should burst out due to the increased probability of fluctuations towards unstable configurations.

Example 1.2 (Volume and thickness in the collapsed case). At small volumes, and in the presence of singularities in the minimizers of $\ell$, collapsing is energetically convenient, and allows $\psi(\varepsilon)$ to approximate $2 \ell$ from below. If $\Gamma \subset \mathbb{R}^{2}$ consists of the three vertexes of an equilateral triangle, for small $\delta$ the unique minimizer of $\ell$ consists of a $Y$-configuration. For small $\varepsilon$, we expect generalized minimizers $(K, E)$ of $\psi(\varepsilon)$ to be collapsed; see Figure 1.3 (b): there, $E$ is a curvilinear triangle made up of three circular arcs whose length is $\mathrm{O}(\sqrt{\varepsilon})$, and whose (negative) curvature is $\mathrm{O}(1 / \sqrt{\varepsilon})$. The thickness of an actual soap film in this configuration should thus be considerably larger near the singularity than along the collapsed region, and the volume and the thickness of the film are somehow independent geometric quantities. This suggests, in the presence of singularities, the need for introducing a second length scale in the model. A possibility is replacing the sharp interface energy $\mathcal{H}^{n}(\Omega \cap \partial E)$ with a diffused interface energy, like the Allen-Cahn energy

$$
\mathcal{E}_{\eta}(u)=\eta \int_{\Omega}|\nabla u|^{2}+\frac{1}{\eta} \int_{\Omega} W(u), \quad \eta>0,
$$

for a double-well potential with $\{W=0\}=\{-1,1\}$. We expect $\{u>0\}$ to (approximately) coincide with the union of a curvilinear triangle of area $\varepsilon$ with three stripes having the collapsed segments as their midsections, and of width $\eta|\log \eta|$; cf. with [19]. 
(a)

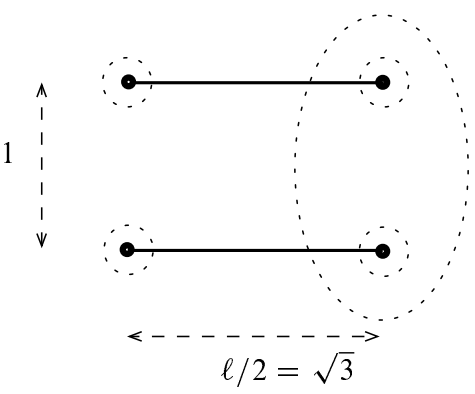

(b)

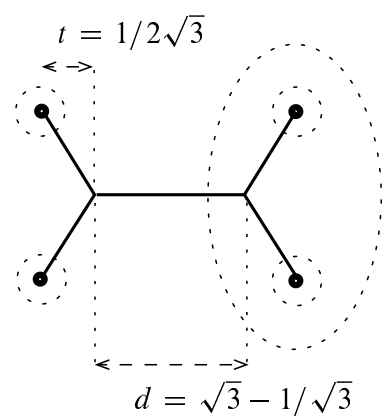

(c)

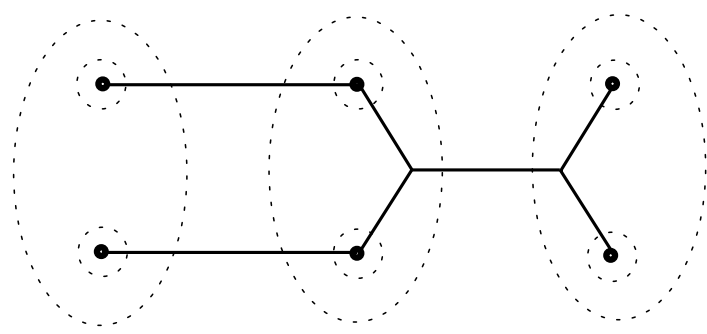

(d)

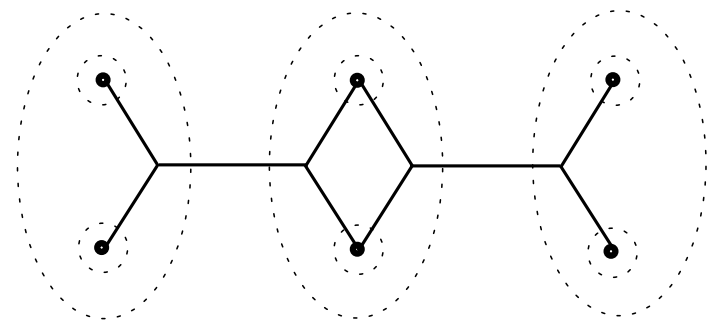

FIGURE 1.4. (a) and (b): A four-points configuration $\Gamma$ with a choice of $\mathcal{C}$ such that $\ell$ admits two minimizers, one with and one without singularities; (c) and (d): a six-points configuration $\Gamma$ with a choice of $\mathcal{C}$ such that $\ell$ admits many minimizers, possibly with a variable number of singularities; here we have depicted two of them, including the one with four singular points that is selected by the $\psi(\varepsilon)$ problems.

Example 1.3 (Capillarity as a selection principle for Plateau's problem). The following statement holds (as a heuristic principle): Generalized minimizers of $\psi(\varepsilon)$ converge to those minimizers of Plateau's problem (1.5) with larger singular set, and when no singular minimizers are present, they select those whose second fundamental form has maximal $L^{2}$-norm. Since the second part of this selection principle is justified by standard second variation arguments, we illustrate the first part only. In Figure 1.4, $\Gamma$ is either given by four or by six points that are suitably spaced so that $\ell$ has different minimizers. As $\varepsilon \rightarrow 0^{+}, \psi(\varepsilon)$ selects those $\ell$-minimizers with singularities over the ones without singularities; and when more minimizers 
with singularities are present, it selects the ones with the largest number of singularities. Indeed, the approximation of a smooth minimizer in $\ell$ will require an energy cost larger than $2 \ell$. At the same time, each time a singularity is present, minimizers of $\psi(\varepsilon)$ can save length in the approximation, thus paying less than $2 \ell$ in energy, and the more the singularities, the bigger the gain. To check this claim, pick $N$ singularities, and denote by $\varepsilon_{i}$ the volume placed near the $i^{\text {th }}$ singularity and by $r_{i}$ the radius of the three circular arcs enclosing $\varepsilon_{i}$. Each wetted singularity has area $c_{1} r_{i}^{2}$, while the total relaxed energy of the approximating configuration is $\mathcal{F}=2 \ell-c_{2} \sum_{i=1}^{N} r_{i}$. Minimizing under the constraint $\varepsilon=c_{1} \sum_{i=1}^{N} r_{i}^{2}$, we must take $r_{i}=\sqrt{\varepsilon / N c_{1}}$, thus finding

$$
\psi(\varepsilon)=2 \ell-c_{2} \sqrt{\frac{\varepsilon N_{\max }}{c_{1}}}
$$

if $N_{\max }$ is the maximal number of singularities available among minimizers of $\ell$. This example suggests that (in every dimension) in the presence of singular minimizers of $\ell$, one should have

$$
\psi^{\prime}(\varepsilon) \rightarrow-\infty \quad \text { as } \varepsilon \rightarrow 0^{+} .
$$

This is of course markedly different from what we expect to be the situation when $\ell$ has only smooth minimizers; see (1.6). We finally notice that a selection principle for the capillarity model (without homotopic spanning conditions) via its AllenCahn approximation has been recently obtained by Leoni and Murray; see [40,41].

\subsection{Statements of the results}

We now give a more technical introduction to our paper, with precise statements, more bibliographical references, and comments on the proofs.

\section{Plateau's problem with homotopic spanning}

We fix a compact set $W \subset \mathbb{R}^{n+1}$ (the "wire frame") and denote the region accessible by the soap film as

$$
\Omega=\mathbb{R}^{n+1} \backslash W
$$

The typical case we have in mind is $W=I_{\delta}(\Gamma)$, as discussed in Section 1.1, but this is not necessary. We fix a spanning class $\mathcal{C}$, that is, a nonempty family of smooth embeddings of $\mathbb{S}^{1}$ into $\Omega$ which is closed by homotopy in $\Omega$. We assume that $W$ and $\mathcal{C}$ are such that the Plateau's problem defined by $\mathcal{C}$

$$
\ell=\inf \left\{\mathcal{H}^{n}(S): S \in \mathcal{S}\right\}
$$

is such that $\ell<\infty !^{4}$ Here, for the sake of brevity, we have introduced

$$
\mathcal{S}=\{S \subset \Omega: S \text { is relatively closed in } \Omega \text { and } S \text { is } \mathcal{C} \text {-spanning } W\} .
$$

\footnotetext{
${ }^{4}$ The condition $\ell<\infty$ clearly implies that no $\gamma \in \mathcal{C}$ is homotopic to a constant map.
} 
As proved in [13, 32], if $\ell<\infty$, then there exists a compact, $\mathcal{H}^{n}$-rectifiable set $S$ such that $\mathcal{H}^{n}(S)=\ell$; see also [10, 12, 15, 18, 23, 24, 28, 31, 33, 35] for related existence results. In addition, $S$ minimizes $\mathcal{H}^{n}$ with respect to Lipschitz perturbations of the identity localized in $\Omega$, so that: (i) $S$ is a classical minimal surface outside of an $\mathcal{H}^{n}$-negligible, relatively closed set in $\Omega$ by [1] ; (ii) if $n=1, S$ consists of finitely many segments, possibly meeting in three equal angles at singular $Y$-points in $\Omega$; (iii) if $n=2, S$ satisfies Plateau's laws by [48]: namely, $S$ is locally diffeomorphic either to a plane or to a cone $Y=T^{1} \times \mathbb{R}$, or to a cone $T^{2}$, where $T^{n}$ is the cone over the origin defined by the $(n-1)$-dimensional faces of a regular tetrahedron in $\mathbb{R}^{n+1}$. The validity of Plateau's laws in this context makes 1.8 more suitable when one is motivated by physical considerations: indeed, minimizers of the codimension one Plateau's problem in the class of rectifiable currents are necessarily smooth if $n \leq 6$. Although smoothness is desirable for geometric applications, it creates an a priori limitation when studying actual soap films; see also [10,13,32].

\section{The capillarity problem and the relaxed energy}

Next, we give a precise formulation of the capillarity problem $\psi(\varepsilon)$ at volume $\varepsilon>0$, which is defined as

$$
\psi(\varepsilon)=\inf \left\{\mathcal{H}^{n}(\Omega \cap \partial E): E \in \mathcal{E},|E|=\varepsilon, \Omega \cap \partial E \text { is } \mathcal{C} \text {-spanning } W\right\} .
$$

Here we have introduced the family of sets

$$
\mathcal{E}=\left\{E \subset \Omega: E \text { is an open set and } \partial E \text { is } \mathcal{H}^{n} \text {-rectifiable }\right\} .
$$

If $E \in \mathcal{E}$, then $\partial E$ is $\mathcal{H}^{n}$-finite and covered by countably many Lipschitz images of $\mathbb{R}^{n}$ into $\mathbb{R}^{n+1}$. Thus, $E$ is of finite perimeter in $\Omega$ by a classical result of Federer, and its (distributional) perimeter $P(E ; U)$ in an open set $U \subset \Omega$ is equal to $\mathcal{H}^{n}\left(U \cap \partial^{*} E\right)$, where $\partial^{*} E$ is the reduced boundary of $E$ (notice that, in general, $\left.P(E ; U) \leq \mathcal{H}^{n}(U \cap \partial E)\right)$. The relaxed energy $\mathcal{F}$ is defined by on every pair $(K, E)$ in the family $\mathcal{K}$ given by

$$
\begin{gathered}
\mathcal{K}=\left\{(K, E): E \subset \Omega \text { is open with } \Omega \cap \operatorname{cl}\left(\partial^{*} E\right)=\Omega \cap \partial E \subset K\right. \\
\left.K \in \mathcal{S} \text { and } K \text { is } \mathcal{H}^{n} \text {-rectifiable in } \Omega\right\} .
\end{gathered}
$$

By the requirement $K \in \mathcal{S}, K$ is $\mathcal{C}$-spanning $W$, while $\Omega \cap \partial E$, which is always a subset of $K$, may not be $\mathcal{C}$-spanning $W$; we expect this when collapsing occurs; see Figure 1.3 .

\section{Assumptions on $\boldsymbol{\Omega}$}

We make two main geometric assumptions on $W$ and $\mathcal{C}$. First, in constructing a system of volume-fixing variations for a given minimizing sequence of $\psi(\varepsilon)$ (see Step 2 of the proof of Theorem 1.4 in Section 3) we shall assume that

$$
\exists \tau_{0}>0 \text { such that, for every } \tau<\tau_{0}, \mathbb{R}^{n+1} \backslash I_{\tau}(W) \text { is connected. }
$$


This is compatible with the idea that, in the physical case $n=2, W$ represents a "solid wire". Second, to verify the finiteness of $\psi(\varepsilon)$ (see Step 1 in the proof of Theorem 1.4, we require that

$$
\exists \eta_{0}>0 \text { and a minimizer } S \text { in } \ell \text { s.t. } \gamma \backslash I_{\eta_{0}}(S) \neq \varnothing \text { for every } \gamma \in \mathcal{C} .
$$

This is clearly a generic situation, which (thanks to the convex hull property of stationary varifolds) is implied, for example, by the much more stringent condition that $\gamma \backslash Z \neq \varnothing$ for every $\gamma \in \mathcal{C}$ where $Z$ is the closed convex hull of $W$. Finally, we shall also assume that " $\partial \Omega=\partial W$ is smooth": by this we mean that locally near each $x \in \partial \Omega, \Omega$ can be described as the epigraph of a smooth function of $n$-variables.

\section{Existence of minimizers and Euler-Lagrange equations}

Our first main result is the existence of generalized minimizers of $\psi(\varepsilon)$.

THEOREM 1.4 (Existence of generalized minimizers). Let $\ell<\infty, \partial W$ be smooth and let (1.11) and (1.12) hold. If $\left\{E_{j}\right\}_{j}$ is a minimizing sequence for $\psi(\varepsilon)$, then there exists a pair $(K, E) \in \mathcal{K}$ with $|E|=\varepsilon$ such that, up to possibly extracting subsequences, and up to possible modifications of each $E_{j}$ outside a large ball containing $W$ (with both operations resulting in defining a new minimizing sequence for $\psi(\varepsilon)$, still denoted by $\left.\left\{E_{j}\right\}_{j}\right)$, we have that

$$
\begin{aligned}
& E_{j} \rightarrow E \quad \text { in } L^{1}(\Omega) \\
& \mathcal{H}^{n}\left\llcorner\left(\Omega \cap \partial E_{j}\right) \stackrel{*}{\rightarrow} \theta \mathcal{H}^{n}\llcorner K \quad \text { as Radon measures in } \Omega\right.
\end{aligned}
$$

as $j \rightarrow \infty$, where $\theta: K \rightarrow \mathbb{R}$ is an upper-semicontinuous function with

$$
\theta=2 \mathcal{H}^{n} \text {-a.e. on } K \backslash \partial^{*} E, \quad \theta=1 \text { on } \Omega \cap \partial^{*} E .
$$

Moreover, $\psi(\varepsilon)=\mathcal{F}(K, E)$ and, for a suitable constant $C$,

$$
\psi(\varepsilon) \leq 2 \ell+C \varepsilon^{n /(n+1)} .
$$

Remark 1.5. Whenever $(K, E) \in \mathcal{K}$ is such that $|E|=\varepsilon, \mathcal{F}(K, E)=\psi(\varepsilon)$, and there exists a minimizing sequence $\left\{E_{j}\right\}_{j}$ for $\psi(\varepsilon)$ that converges to $(K, E)$ as in (1.13), we say that $(K, E)$ is a generalized minimizer of $\psi(\varepsilon)$. We say that $(K, E)$ is collapsed if $K \backslash \partial E \neq \varnothing$. If $(K, E)$ is not collapsed, then $E$ is a (standard) minimizer of $\psi(\varepsilon)$.

Next, we derive the Euler-Lagrange equations for a generalized minimizer and apply Allard's theorem.

THEOREM 1.6 (Euler-Lagrange equation for generalized minimizers). Let $\ell<\infty$, $\partial W$ be smooth, and let (1.11) and (1.12) hold. If $(K, E)$ is a generalized minimizer of $\psi(\varepsilon)$ and $f: \Omega \rightarrow \Omega$ is a diffeomorphism such that $|f(E)|=|E|$, then

$$
\mathcal{F}(K, E) \leq \mathcal{F}(f(K), f(E)) .
$$

In particular, 
(i) there exists $\lambda \in \mathbb{R}$ such that

$\lambda \int_{\partial^{*} E} X \cdot v_{E} d \mathcal{H}^{n}=\int_{\partial^{*} E} \operatorname{div}^{K} X d \mathcal{H}^{n}+2 \int_{K \backslash \partial^{*} E} \operatorname{div}^{K} X d \mathcal{H}^{n}$

for every $X \in C_{c}^{1}\left(\mathbb{R}^{n+1} ; \mathbb{R}^{n+1}\right)$ with $X \cdot v_{\Omega}=0$ on $\partial \Omega$, where $\operatorname{div}^{K}$ denotes the tangential divergence along $K$;

(ii) there exists $\Sigma \subset K$, closed and with empty interior in $K$, such that $K \backslash \Sigma$ is a smooth hypersurface, $K \backslash(\Sigma \cup \partial E)$ is a smooth embedded minimal hypersurface, $\mathcal{H}^{n}(\Sigma \backslash \partial E)=0, \Omega \cap\left(\partial E \backslash \partial^{*} E\right) \subset \Sigma$ has empty interior in $K$, and $\Omega \cap \partial^{*} E$ is a smooth embedded hypersurface with constant scalar $\left(\right.$ w.r.t. $\left.v_{E}\right)$ mean curvature $\lambda$.

Remark 1.7. Although we do not pursue this point here, we mention that we would expect $(K, E)$ to be a proper minimizer of $\mathcal{F}$ among pairs $\left(K^{\prime}, E^{\prime}\right) \in \mathcal{K}$ with $\left|E^{\prime}\right|=\varepsilon$ (and not just when $K^{\prime}=f(K)$ for a diffeomorphism $f$, as proved in (1.15)). To show this we would need to approximate in energy a generic $\left(K^{\prime}, E^{\prime}\right)$ by competitors $\left\{F_{j}\right\}_{j}$ for $\psi(\varepsilon)$. The natural ansatz for this approximation would be taking $F_{j}=U_{\eta_{j}}\left(K^{\prime} \cup E^{\prime}\right) \backslash I_{\eta_{j}}\left(K^{\prime} \cap E^{\prime}\right)$ for $\eta_{j} \rightarrow 0^{+}$, where $U_{\eta}$ denotes the open $\eta$-neighborhood of a set. The convergence of this approximation is delicate, and can be made to work by elaborating on the ideas contained in [2, 49] at least for $\left(K^{\prime}, E^{\prime}\right)$ in certain subclasses of $\mathcal{K}$.

Remark 1.8. Theorem 1.6 points at two interesting free boundary problems. The first problem concerns the size and properties of $\partial E \backslash \partial^{*} E$, which is the transition region between constant and zero mean curvature; similar free boundary problems (on graphs rather than on unconstrained surfaces) have been considered, e.g., in [6-8]. The second problem concerns the wetted region $\partial \Omega \cap \partial E$, which could either be $\mathcal{H}^{n}$-negligible or not; recall Figure 1.3 in the former case, $\partial \Omega \cap \partial E$ should be $(n-1)$-dimensional, while in the latter case $\partial \Omega \cap \partial E$ should be a set of finite perimeter inside $\partial \Omega$, and Young's law $v_{\Omega} \cdot v_{E}=0$ should hold at generic boundary points of $\partial \Omega \cap \partial E$ relative to $\partial \Omega$; see, for example, [16, 17].

\subsection{Convergence towards Plateau's problem}

The next theorem establishes the nature of Plateau's problem $\ell$ as the singular limit of the capillarity problems $\psi(\varepsilon)$ as $\varepsilon \rightarrow 0^{+}$.

THEOREM 1.9 (Plateau's problem as a singular limit of capillarity problems). If $\ell<\infty, \partial W$ is smooth, and (1.11) and (1.12) hold, then $\psi$ is lower-semicontinuous on $(0, \infty)$ and

$$
\lim _{\varepsilon \rightarrow 0^{+}} \psi(\varepsilon)=2 \ell
$$

In addition, if $\left\{\left(K_{h}, E_{h}\right)\right\}_{h}$ is a sequence of generalized minimizers of $\psi\left(\varepsilon_{h}\right)$ for $\varepsilon_{h} \rightarrow 0^{+}$as $h \rightarrow \infty$, then there exists a minimizer $S$ in $\ell$ such that, up to extracting subsequences and as $h \rightarrow \infty$,

$$
\mathcal{H}^{n}\left\llcorner\left(\Omega \cap \partial^{*} E_{h}\right)+2 \mathcal{H}^{n}\left\llcorner\left(K_{h} \backslash \partial^{*} E_{h}\right) \stackrel{*}{\rightarrow} 2 \mathcal{H}^{n}\llcorner S\right.\right.
$$



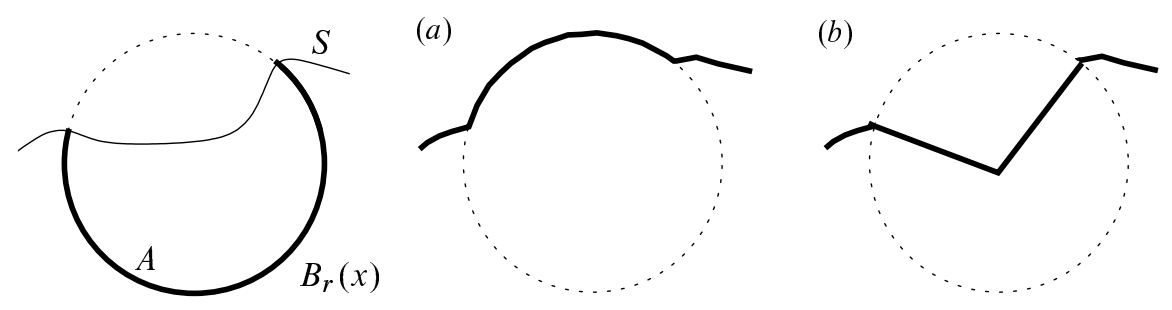

FIgURE 1.5. (a) The cup competitor of a set $S$ in $B_{r}(x)$ relative to an $\mathcal{H}^{n}$-maximal connected component $A$ of $\partial B_{r}(x) \backslash S$; (b) the cone competitor of $S$ in $B_{r}(x)$.

as Radon measures in $\Omega$.

Remark 1.10. The behavior of $\psi(\varepsilon)-2 \ell$ as $\varepsilon \rightarrow 0^{+}$is expected to depend heavily on whether minimizers of $\ell$ have or do not have singularities, as noticed in (1.6) and (1.7). In particular, we expect $\psi^{\prime}(\varepsilon) \rightarrow 0^{+}$only in special situations: when this happens, we have a vanishing mean curvature approximation of Plateau's problem that is related to Rellich's conjecture; see, e.g., [5].

Remark 1.11. The Hausdorff convergence of $K_{h}$ to $S$ is not immediate (nor is the convergence in the varifolds sense). Given (1.18), Hausdorff convergence would follow from an area lower bound on $K_{h}$. In turn, this could be deduced (thanks to area monotonicity) from a uniform $L^{p}$-bound, for some $p>n$, on the mean curvature vectors $\vec{H}_{V_{h}}$ of the integer varifolds $V_{h}$ supported on $K_{h}$, with multiplicity 2 on $K_{h} \backslash \partial^{*} E_{h}$, and multiplicity 1 on $\partial^{*} E_{h}$. Notice however that, by (1.16), if $\lambda_{h}$ is the Lagrange multiplier of $\left(K_{h}, E_{h}\right)$, then $\vec{H}_{V_{h}}=\lambda_{h} v_{E_{h}} 1_{\partial^{*} E_{h}}$, so that, even when $n=1$, the only uniform $L^{p}$-bound that can hold is the one with $p=1$; see Example 1.2 .

\section{Proofs}

We approach Theorem 1.4 with the method introduced in [13] to solve (1.8), which is now briefly summarized. The idea in [13] is to consider a minimizing sequence $\left\{S_{j}\right\}_{j}$ for $\ell$, which (up to extracting subsequences) immediately leads to a sequence of Radon measures $\mu_{j}=\mathcal{H}^{n}\left\llcorner S_{j} \stackrel{*}{\rightarrow} \mu\right.$ as Radon measures in $\Omega$, with $S=\operatorname{spt} \mu \mathcal{C}$-spanning $W$. By comparing $S_{j}$ with its cup competitors $S_{j}^{\prime}$ (see Figure 1.5(a)), and then letting $j \rightarrow \infty$, it is shown that $\mu\left(B_{r}(x)\right) \geq \theta_{0}(n) r^{n}$ for every $x \in \operatorname{spt} \mu$; by comparing $S_{j}$ with its cone competitors $S_{j}^{\prime}$, and then letting $j \rightarrow \infty$, it is proved that $r^{-n} \mu\left(B_{r}(x)\right)$ is increasing in $r$. By Preiss' theorem [11 46] it follows that $\mu=\theta \mathcal{H}^{n}\left\llcorner S\right.$ and that $S$ is $\mathcal{H}^{n}$-rectifiable. Finally, spherical isoperimetry and a geometric argument imply that $\theta \geq 1 \mathcal{H}^{n}$-a.e. on $S$, which in turn suffices to conclude that $S$ is a minimizer in $\ell$ since, by lower semicontinuity, $\mathcal{H}^{n}(S) \leq \mu(\Omega) \leq \liminf _{j} \mu_{j}(\Omega)=\ell$, and because $S$ is in the competition class of $\ell$. 
Adapting this approach to a minimizing sequence $\left\{E_{j}\right\}_{j}$ for $\psi(\varepsilon)$ requires the introduction of new ideas. First, cup and cone competitors for $\left\{E_{j}\right\}_{j}$ have to be defined as boundaries, a feature that requires taking into consideration two kinds of cup competitors, and that also leads to other difficulties. Second, local variations need to be compensated by volume-fixing variations, which must be uniform along the elements of the minimizing sequence. At this stage, we can prove that $\mu_{j}=\mathcal{H}^{n}\left\llcorner\left(\Omega \cap \partial E_{j}\right) \stackrel{*}{\rightarrow} \mu=\theta \mathcal{H}^{n}\left\llcorner K\right.\right.$ for an $\mathcal{H}^{n}$-rectifiable set $K$ that is $\mathcal{C}$ spanning $W$. The same argument as in [13] shows that $\theta \geq 1$, and the lower bound $\theta \geq 2 \mathcal{H}^{n}$-a.e. on $K \backslash \partial^{*} E$ requires a further elaboration which takes into account that we are considering the convergence of boundaries. We cannot conclude that $\mathcal{F}(K, E)=\psi(\varepsilon)$ just by lower semicontinuity because clearly $(K, E)$ is not in the competition class of $\psi(\varepsilon)$. We thus improve lower semicontinuity by some nonconcentration estimates: at infinity, at the boundary, and by folding against $K$. The latter are the most interesting ones, and they require a careful comparison argument based on the introduction of a third kind of competitors, called slab competitors. The construction of the various competitors is discussed in Section 2 , while the proof of Theorem 1.4 is contained in Section 3. Slab competitors are also used in the delicate proof of (1.15), whose starting point are some ideas originating in [14], as further developed in [13] when addressing the formulation of Plateau's problem for David's sliding minimizers; see Section 4 Finally, in Section 5 we prove Theorem 1.9, the main difficulty, explained there in more detail, is that, at vanishing volume, we have no nontrivial local limit sets to be used for constructing uniform volume-fixing variations.

\section{Structure of generalized minimizers}

Theorem 1.4, Theorem 1.6, and Theorem 1.9 lay the foundations to study the properties of generalized minimizers of $\psi(\varepsilon)$. The most intriguing questions are concerned with the relations between the properties of minimizers in Plateau's problem $\ell$, like the presence or the absence of singularities, and the properties of minimizers in $\psi(\varepsilon)$ at small $\varepsilon$ : collapsing versus noncollapsing and the sign of $\lambda$, limiting behavior of $\lambda$ as $\varepsilon \rightarrow 0^{+}$, dimensionality of the wetted part of the wire, etc. This is of course a very large set of problems, which will require further investigations. In the companion paper [37], we start this kind of study by proving that collapsed minimizers have nonpositive Lagrange multipliers, deduce from this property that they satisfy the convex hull property, and lay the ground for the forthcoming paper [38], where we further investigate the regularity of the collapsed set $K \backslash \partial^{*} E$.

\section{Cone, cup, and slab competitors, nucleation, and collapsing}

Section 2.1 contains the notation and terminology used in the paper. Section 2.2 collects some basic properties of $\mathcal{C}$-spanning sets. Sections 2.3, 2.4 and 2.5 deal 
with cup, slab, and cone competitors. Section 2.6 contains the nucleation lemma for volume-fixing variations, and Section 2.7 concerns density lower bounds for collapsing sequences of sets of finite perimeter.

\subsection{Notation and terminology}

We denote by $|A|$ and $\mathcal{H}^{s}(A)$ the Lebesgue and the $s$-dimensional Hausdorff measures of $A \subset \mathbb{R}^{n+1}$, by $I_{\eta}(A)$ and $U_{\eta}(A)$ the closed and open $\eta$-neighborhoods of $A$, and by $B_{r}(x)$ the open ball of center at $x$ and radius $r$. We work in the framework of [3, 42,47]. Given $k \in \mathbb{N}, 1 \leq k \leq n$, a Borel set $M \subset \mathbb{R}^{n+1}$ is countably $\mathcal{H}^{k}$-rectifiable if it is covered by countably many Lipschitz images of $\mathbb{R}^{k}$; it is (locally) $\mathcal{H}^{k}$-rectifiable if, in addition, $M$ is (locally) $\mathcal{H}^{k}$-finite. If $M$ is locally $\mathcal{H}^{k}$-rectifiable, then for $\mathcal{H}^{k}$-a.e. $x \in M$ there exists a unique $k$-plane $T_{x} M$ such that, as $r \rightarrow 0^{+}, \mathcal{H}^{k}\left\llcorner(M-x) / r \stackrel{*}{\rightarrow} \mathcal{H}^{k}\left\llcorner T_{x} M\right.\right.$ as Radon measures in $\mathbb{R}^{n+1} ; T_{x} M$ is called the approximate tangent plane to $M$ at $x$. Given a Lipschitz map $f: \mathbb{R}^{n+1} \rightarrow \mathbb{R}^{n+1}$, we denote by $J^{M} f$ its tangential Jacobian along $M$, so that if $f$ is smooth and $f(x)=x+t X(x)+\mathrm{o}(t)$ in $C^{1}$ as $t \rightarrow 0^{+}$, then $J^{M} f=$ $1+t \operatorname{div}^{M} X+\mathrm{o}(t)$ where $\operatorname{div}^{M} X$ is the tangential divergence of $X$ along $M$; moreover, $M$ has distributional mean curvature vector $\vec{H} \in L_{\mathrm{loc}}^{1}\left(U ; \mathcal{H}^{k}\llcorner M)\right.$ in $U$ open, if

$$
\int_{M} \operatorname{div}^{M} X d \mathcal{H}^{k}=\int_{M} X \cdot \vec{H} d \mathcal{H}^{k} \quad \forall X \in C_{c}^{\infty}\left(U ; \mathbb{R}^{n+1}\right)
$$

see [47, secs. 8 and 9]. A Borel set $E \subset \mathbb{R}^{n+1}$ has finite perimeter if there exists an $\mathbb{R}^{n+1}$-valued Radon measure on $\mathbb{R}^{n+1}$, denoted by $\mu_{E}$, such that $\left\langle\mu_{E}, X\right\rangle=$ $\int_{E} \operatorname{div} X$ whenever $X \in C_{c}^{1}\left(\mathbb{R}^{n+1} ; \mathbb{R}^{n+1}\right)$ and $P\left(E ; \mathbb{R}^{n+1}\right)=\left|\mu_{E}\right|\left(\mathbb{R}^{n+1}\right)<$ $\infty$. The set of points $x \in \mathbb{R}^{n+1}$ such that

$$
\frac{\mu_{E}\left(B_{r}(x)\right)}{\left|\mu_{E}\right|\left(B_{r}(x)\right)} \rightarrow v_{E}(x) \in \mathbb{S}^{n} \quad \text { as } r \rightarrow 0^{+}
$$

is denoted by $\partial^{*} E$ and called the reduced boundary $\partial^{*} E$ of $E$. Then $\mu_{E}=$ $v_{E} \mathcal{H}^{n}\left\llcorner\partial^{*} E, \partial^{*} E\right.$ is $\mathcal{H}^{n}$-rectifiable in $\mathbb{R}^{n+1}$, and $T_{x} \partial^{*} E=v_{E}(x)^{\perp}$ for every $x \in \partial^{*} E$. The set $E^{(t)}$ of points of density $t \in[0,1]$ of $E$ is given by those $x \in \mathbb{R}^{n+1}$ with $\left|E \cap B_{r}(x)\right| /\left|B_{r}(x)\right| \rightarrow t$ as $r \rightarrow 0^{+}$, and (see, e.g., [42, theorem 16.2]),

$$
\left\{\partial^{*} E, E^{(0)}, E^{(1)}\right\} \quad \text { is a partition of } \mathbb{R}^{n+1} \text { modulo } \mathcal{H}^{n} .
$$

Federer's criterion [25, 4.5.11] states that if the essential boundary $\partial^{\mathrm{e}} E=\mathbb{R}^{n+1} \backslash$ $\left(E^{(0)} \cup E^{(1)}\right)$ is $\mathcal{H}^{n}$-finite, then $E$ is of finite perimeter in $\mathbb{R}^{n+1}$. If $E$ is open, then $\partial^{\mathrm{e}} E \subset \partial E$ : hence, if $E \in \mathcal{E}$ and $\mathcal{H}^{n}(\partial \Omega)<\infty$, then $E$ is of finite perimeter.

\subsection{Some preliminary results}

In the following, $W$ is a compact set, $\mathcal{C}$ a spanning class for $W$, and $\Omega=$ $\mathbb{R}^{n+1} \backslash W$. 
LEMMA 2.1. If $\left\{K_{j}\right\}_{j}$ are relatively closed sets in $\Omega$ such that each $K_{j}$ is $\mathcal{C}$ spanning $W$ and $\mathcal{H}^{n}\left\llcorner K_{j} \stackrel{*}{\rightarrow} \mu\right.$ as Radon measures in $\Omega$, then $K=\Omega \cap \operatorname{spt} \mu$ is $\mathcal{C}$-spanning $W$.

Proof. See [13, step 2, proof of theorem 4].

Lemma 2.2. Let $K$ be relatively closed in $\Omega$ and let $B_{r}(x) \Subset \Omega$. Then $K$ is $\mathcal{C}$-spanning $W$ if and only if, whenever $\gamma \in \mathcal{C}$ is such that $\gamma \cap K \backslash B_{r}(x)=\varnothing$, then there exists a connected component of $\gamma \cap \operatorname{cl}\left(B_{r}(x)\right)$ that is diffeomorphic to an interval, and whose endpoints belong to distinct connected components of $\operatorname{cl}\left(B_{r}(x)\right) \backslash K$, as well as to distinct components of $\partial B_{r}(x) \backslash K$.

Proof. This is [13, lemma 10].

LemmA 2.3. If $K$ is $\mathcal{C}$-spanning $W, B_{r}(x) \Subset \Omega$, and $f: \mathbb{R}^{n+1} \rightarrow \mathbb{R}^{n+1}$ is a bi-Lipschitz map with $\{f \neq \mathrm{id}\} \Subset B_{r}(x)$ and $f\left(B_{r}(x)\right) \subset B_{r}(x)$, then $f(K)$ is $\mathcal{C}$-spanning $W$.

Proof. By $f(K) \backslash B_{r}(x)=K \backslash B_{r}(x)$, if $f(K)$ is not $\mathcal{C}$-spanning $W$, then there exists $\gamma \in \mathcal{C}$ with $\gamma \cap K \backslash B_{r}(x)=\varnothing$ such that $\gamma \cap f(K)=\varnothing$. Hence, the curve $\tilde{\gamma}:=f^{-1} \circ \gamma$ is a continuous embedding of $\mathbb{S}^{1}$ in $\Omega$, homotopic to $\gamma$ in $\Omega$, and such that $\tilde{\gamma} \cap K=\varnothing$. Since $\tilde{\gamma}$ and $W$ are compact and $K$ is closed, $\tilde{\gamma}$ has positive distance from $K \cup W$, and by smoothing out $\tilde{\gamma}$ we define a smooth embedding $\hat{\gamma}$ of $\mathbb{S}^{1}$ into $\Omega$, disjoint from $K$, and homotopic to $\tilde{\gamma}$ (and therefore to $\gamma)$ in $\Omega$, a contradiction.

LEMMA 2.4. If $\partial \Omega$ is smooth, then there exists $r_{0}>0$ with the following property. If $x \in \partial \Omega, \Omega \subset \Omega^{\prime}, f: \operatorname{cl}(\Omega) \rightarrow \operatorname{cl}\left(\Omega^{\prime}\right)=f(\operatorname{cl}(\Omega))$ is a homeomorphism with $f(\partial \Omega)=\partial \Omega^{\prime},\{f \neq \mathrm{id}\} \Subset B_{r_{0}}(x)$, and $f\left(B_{r_{0}}(x) \cap \operatorname{cl}(\Omega)\right)=B_{r_{0}}(x) \cap \operatorname{cl}\left(\Omega^{\prime}\right)$, and if $K$ is $\mathcal{C}$-spanning $W$, then $K^{\prime}=f\left(K \cap \Omega^{*}\right)$ is relatively closed in $\Omega$ and is $\mathcal{C}$-spanning $W$, where $\Omega^{*}=f^{-1}(\Omega)$.

\section{PROOF.}

Step 1 . We show that, for $K$ relatively closed in $\Omega$ and $B_{r_{0}}(x)$ as in the statement, $K$ is $\mathcal{C}$-spanning $W$ if and only if, whenever $\gamma \in \mathcal{C}$ is such that $\gamma \cap K \backslash$ $B_{r_{0}}(x)=\varnothing$, then there exists a connected component of $\gamma \cap \operatorname{cl}\left(B_{r_{0}}(x)\right)$, diffeomorphic to an interval, and whose endpoints belong to distinct connected components of $\Omega \cap \operatorname{cl}\left(B_{r_{0}}(x)\right) \backslash K$.

We only prove the "only if" part. First of all, we notice that $\gamma$ cannot be contained in $\Omega \cap B_{r_{0}}(x)$, because $r_{0}$ can be chosen small enough to ensure that $\Omega \cap B_{r_{0}}(x)$ is simply connected, and because $\ell<\infty$ implies that no element of $\mathcal{C}$ is homotopic to a constant. Arguing as in [13, step two, proof of lemma 10], we can assume that $\gamma$ and $\partial B_{r_{0}}(x)$ intersect transversally, so that there exist finitely many disjoint $I_{i}=\left[a_{i}, b_{i}\right] \subset \mathbb{S}^{1}$ such that $\gamma \cap \operatorname{cl}\left(B_{r_{0}}(x)\right)=\bigcup_{i} \gamma\left(I_{i}\right)$ with $\gamma \cap \partial B_{r_{0}}(x)=\bigcup_{i}\left\{\gamma\left(a_{i}\right), \gamma\left(b_{i}\right)\right\}$ and $\gamma \cap B_{r_{0}}(x)=\bigcup_{i} \gamma\left(\left(a_{i}, b_{i}\right)\right)$. Assume by contradiction that for each $i$ there exists a connected component $A_{i}$ of 
$\Omega \cap \operatorname{cl}\left(B_{r_{0}}(x)\right) \backslash K$ such that $\gamma\left(a_{i}\right), \gamma\left(b_{i}\right) \in A_{i}$. If $r_{0}$ is small enough, then $\operatorname{cl}\left(\Omega \cap B_{r_{0}}(x)\right)$ is diffeomorphic to $\operatorname{cl}\left(B_{1}(0) \cap\left\{x_{1}>0\right\}\right)$ through a diffeomorphism mapping $B_{r_{0}}(x) \cap \partial \Omega$ into $B_{1}(0) \cap\left\{x_{1}=0\right\}$. Using this fact and the connectedness of each $A_{i}$, we define smooth embeddings $\tau_{i}: I_{i} \rightarrow A_{i}$ with $\tau_{i}\left(a_{i}\right)=\gamma\left(a_{i}\right)$, $\tau_{i}\left(b_{i}\right)=\gamma\left(b_{i}\right)$, and $\tau_{i}$ homotopic in $\Omega \cap B_{r_{0}}(x)$ to the restriction of $\gamma$ to $I_{i}$. Moreover, this can be done with $\tau_{i}\left(I_{i}\right) \cap \tau_{j}\left(I_{j}\right)=\varnothing$. The new embedding $\bar{\gamma}$ of $\mathbb{S}^{1}$ obtained by replacing $\gamma$ with $\tau_{i}$ on $I_{i}$ is thus homotopic to $\gamma$ in $\Omega$ and such that $\bar{\gamma} \cap K=\varnothing$, a contradiction.

Step 2. Since $K \cap \Omega^{*}$ is relatively closed in $\Omega^{*}, K^{\prime}=f\left(K \cap \Omega^{*}\right)$ is relatively closed in $\Omega=f\left(\Omega^{*}\right)$. Should $K^{\prime}$ not be $\mathcal{C}$-spanning $W$, given that $K^{\prime} \backslash B_{r_{0}}(x)=$ $K \backslash B_{r_{0}}(x)$, we could find $\gamma \in \mathcal{C}$ with $\gamma \cap K \backslash B_{r_{0}}(x)=\varnothing$ and $\gamma \cap K^{\prime}=\varnothing$. By Step 1, there would be a connected component $\sigma$ of $\gamma \cap \operatorname{cl}\left(B_{r_{0}}(x)\right)$, diffeomorphic to an interval, and such that: (i) the endpoints $p$ and $q$ of $\sigma$ (which lie on $\partial B_{r_{0}}(x)$ ) belong to distinct connected components of $\Omega \cap \operatorname{cl}\left(B_{r_{0}}(x)\right) \backslash K$; and (ii) $p$ and $q$ belong to the same connected component of $\Omega \cap \operatorname{cl}\left(B_{r_{0}}(x)\right) \backslash K^{\prime}$. Since $f$ is a homeomorphism, $f(p)=p$, and $f(q)=q$, by (i) we would find that $p$ and $q$ belong to distinct connected components of

$$
f\left(\Omega \cap \operatorname{cl}\left(B_{r_{0}}(x)\right) \backslash K\right)=\Omega^{\prime} \cap \operatorname{cl}\left(B_{r_{0}}(x)\right) \backslash f(K),
$$

while, by (ii), there would be an arc connecting $p$ and $q$ in $\Omega \cap \operatorname{cl}\left(B_{r_{0}}(x)\right) \backslash K^{\prime}$, where

$$
\begin{aligned}
\Omega \cap \operatorname{cl}\left(B_{r_{0}}(x)\right) \backslash K^{\prime} & =\Omega \cap \operatorname{cl}\left(B_{r_{0}}(x)\right) \backslash f\left(K \cap \Omega^{*}\right) \\
& =\Omega \cap \operatorname{cl}\left(B_{r_{0}}(x)\right) \backslash f(K) \subset \Omega^{\prime} \cap \operatorname{cl}\left(B_{r_{0}}(x)\right) \backslash f(K)
\end{aligned}
$$

and hence $p$ and $q$ would belong to a same component of $\Omega^{\prime} \cap \operatorname{cl}\left(B_{r_{0}}(x)\right) \backslash f(K)$.

\subsection{Cup competitors}

Given $E \in \mathcal{E}, B_{r}(x) \Subset \Omega$ and a connected component $A$ of $\partial B_{r}(x) \backslash \partial E$, cup competitors are used to compare $\mathcal{H}^{n}\left(B_{r}(x) \cap \partial E\right)$ with $\mathcal{H}^{n}\left(\partial B_{r}(x) \backslash A\right)$. The construction is more involved than in the case of Plateau's problem considered in [13] as we need to construct cup competitors as boundaries, and we have to argue differently depending on whether $A \cap E=\varnothing$ or $A \subset E$.

LEMma 2.5 (Cup competitors). Let $E \in \mathcal{E}$ be such that $\Omega \cap \partial E$ is $\mathcal{C}$-spanning $W$, let $x \in \Omega, 0<r<\operatorname{dist}(x, \partial \Omega)$, and let $A$ be a connected component of $\partial B_{r}(x) \backslash$ $\partial E$. Assume that $\partial E \cap \partial B_{r}(x)$ is $\mathcal{H}^{n-1}$-rectifiable. Then, for every $\eta \in(0, r / 2)$ there exists a set $F=F_{\eta} \in \mathcal{E}$ so that $\Omega \cap \partial F$ is $\mathcal{C}$-spanning $W$, and

$$
\begin{aligned}
& \partial F \backslash \operatorname{cl}\left(B_{r}(x)\right)=\partial E \backslash \operatorname{cl}\left(B_{r}(x)\right), \\
& \lim _{\eta \rightarrow 0^{+}} \mathcal{H}^{n}\left(\left(\partial B_{r}(x) \cap \partial F\right) \Delta\left(\partial B_{r}(x) \backslash A\right)\right)=0,
\end{aligned}
$$


(a)
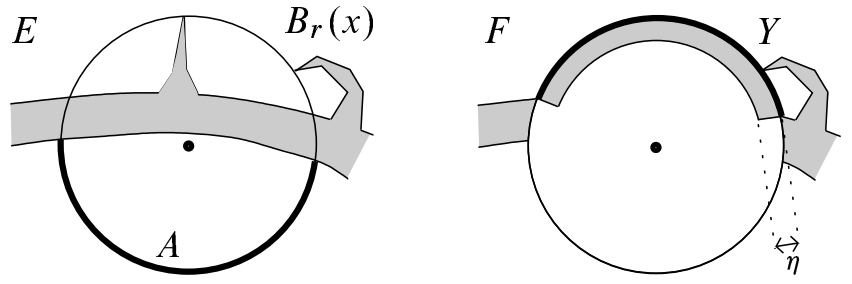

(b)
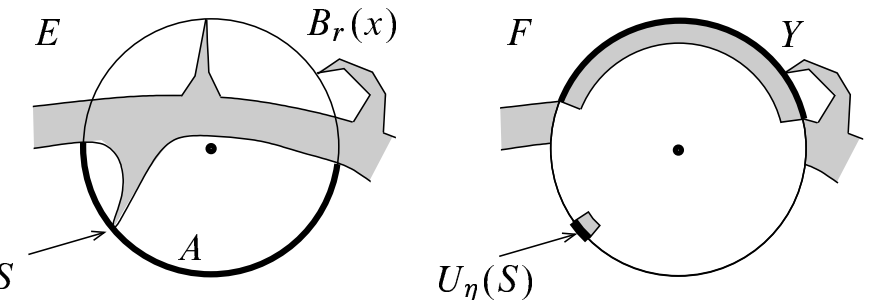

(c)
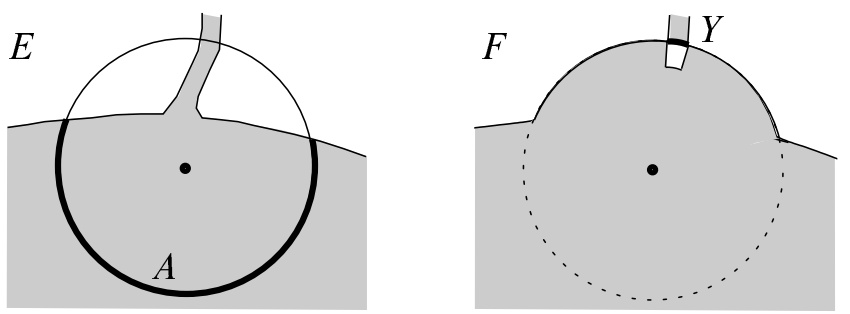

FIGURE 2.1. Cup competitors when: (a) $A \cap E=\varnothing$ and $S=\varnothing$; (b) $A \cap E=\varnothing$ and $S \neq \varnothing$; (c) $A \subset E$. Picture (b) really pertains to the case $n \geq 2$, in which the component $A$ in the picture is not necessarily disconnected by the presence of $S$. In the situation of picture (b) the set $F$ defined by (2.9) may fail to intersect a test curve $\gamma$ which was intersecting with $\Omega \cap \partial E$ only at points in $S$.

$$
\limsup _{\eta \rightarrow 0^{+}} \mathcal{H}^{n}(\Omega \cap \partial F) \leq \mathcal{H}^{n}\left(\Omega \cap \partial E \backslash B_{r}(x)\right)+2 \mathcal{H}^{n}\left(\partial B_{r}(x) \backslash A\right) .
$$

Moreover,

(i) If $A \cap E=\varnothing$, then

$$
\limsup _{\eta \rightarrow 0^{+}} \mathcal{H}^{n}\left(B_{r}(x) \cap \partial F\right) \leq \mathcal{H}^{n}\left(\partial B_{r}(x) \backslash\left(A \cup\left(E \cap \partial B_{r}\right)\right)\right) ;
$$

(ii) If $A \subset E$, then

$$
\limsup _{\eta \rightarrow 0^{+}} \mathcal{H}^{n}\left(B_{r}(x) \cap \partial F\right) \leq \mathcal{H}^{n}\left(E \cap \partial B_{r}(x) \backslash A\right) .
$$

Remark 2.6. Before proceeding with the proof of the lemma, let us first provide some additional details on the construction of the competitors $F=F_{\eta}$, which, as 
anticipated, is different depending on whether $A \cap E=\varnothing$ or $A \subset E$. In what follows, given $Y \subset \partial B_{r}(x)$, we set

$$
N_{\eta}(Y)=\left\{y-t v_{B_{r}(x)}(y): y \in Y, t \in(0, \eta)\right\}, \quad 0<\eta<r .
$$

Case $A \cap E=\varnothing$. In this case, we define

$$
Y=\partial B_{r}(x) \backslash\left(\operatorname{cl}\left(E \cap \partial B_{r}(x)\right) \cup \operatorname{cl}(A)\right),
$$

and then we further distinguish two scenarios, depending on whether the set

$$
S=\partial E \cap \operatorname{cl}(A) \backslash\left[\operatorname{cl}\left(E \cap \partial B_{r}(x)\right) \cup \operatorname{cl}(Y)\right]
$$

is empty or not. When $S=\varnothing$ the cup competitor defined by $E$ and $A$ is given by

$$
F=\left(E \backslash \operatorname{cl}\left(B_{r}(x)\right)\right) \cup N_{\eta}(Y) ;
$$

see Figure 2.1-(a) and Step 1 of the proof. When $S \neq \varnothing$ (see Figure 2.1-(b)), if we define $F$ as in (2.9), then $\Omega \cap \partial F$ may fail to be $\mathcal{C}$-spanning $W$; we thus need to modify (2.9), and to this end, denoting by $\mathrm{d}_{S}$ the distance function from $S$ and by $U_{\eta}(S)=\partial B_{r}(x) \cap\left\{\mathrm{d}_{S}(y)<\eta\right\}$, we set

(2.10) $F=\left(E \backslash \operatorname{cl}\left(B_{r}(x)\right)\right) \cup N_{\eta}(Z), \quad Z=Y \cup\left(U_{\eta}(S) \backslash \operatorname{cl}\left(E \cap \partial B_{r}(x)\right)\right)$;

see, again, Figure 2.1.(b). This situation, discussed in detail in Step 2 of the proof, is made more delicate since we can prove that the sets defined in (2.10) are wellbehaved in the limit as $\eta \rightarrow 0^{+}$only along a suitable sequence $\eta_{k} \downarrow 0^{+}$. For this reason, we will actually define $F_{\eta}$ as in 2.10 only when $\eta=\eta_{k}$, and then extend the definition by setting $F_{\eta}=F_{\eta_{k}}$ for all $\eta \in\left(\eta_{k+1}, \eta_{k}\right)$ (so that, for the sake of homogenity, 2.4 can be stated as an $\eta \rightarrow 0^{+}$-limit in all three cases).

Case $A \cap E=\varnothing$. Finally, when $A \subset E$ the cup competitor defined by $E$ and $A$ is given by

$$
F=\left(E \cup B_{r}(x)\right) \backslash \operatorname{cl}\left(N_{\eta}(Y)\right), \quad Y=\left(E \cap \partial B_{r}(x)\right) \backslash \operatorname{cl}(A) ;
$$

see Figure 2.1 (c). We treat this case in Step 3 of the proof.

PROOF.

Step 1. We assume that $A \cap E=\varnothing$ and, after defining $Y$ as in (2.7) and $S$ as in (2.8), we suppose first that

$$
S=\varnothing
$$

We then define $F$ by (2.9). For the sake of brevity we set $B_{r}=B_{r}(x)$. We claim that 2.2 holds, and that we have

$$
\begin{aligned}
B_{r} \cap \partial F & =B_{r} \cap \partial N_{\eta}(Y), \\
Y & \subset \partial F \cap \partial B_{r}, \\
E \cap \partial B_{r} & \subset \partial F \cap \partial B_{r}, \\
\partial B_{r} \backslash \operatorname{cl}(A) & \subset \partial F \cap \partial B_{r},
\end{aligned}
$$


Indeed, (2.2) and (2.13) follow from $F \cap B_{r}=N_{\eta}(Y) \cap B_{r}$ and $F \backslash \operatorname{cl}\left(B_{r}\right)=$ $E \backslash \operatorname{cl}\left(B_{r}\right)$. To prove (2.14): $Y \subset \operatorname{cl}\left(N_{\eta}(Y)\right)$ gives $Y \subset \operatorname{cl}(F)$, and $F \cap \partial B_{r}=\varnothing$ implies $Y \cap F=\varnothing$. To prove (2.15): $E \cap \partial B_{r} \subset \operatorname{cl}\left(E \backslash \operatorname{cl}\left(B_{r}\right)\right)$, so that $E \cap \partial B_{r} \subset$ $\operatorname{cl}(F)$, while $F \cap \partial B_{r}=\varnothing$ gives $\left(E \cap \partial B_{r}\right) \cap F=\varnothing$. (2.18) is obvious, and (2.16) follows from (2.14) and (2.15). (2.17) is then an immediate consequence of (2.14), (2.15), 2.16), and the condition in (2.12). To prove (2.19): $A$ is open in $\partial B_{r} \backslash \partial E$ and $A \cap E=\varnothing$, thus $A \cap \operatorname{cl}(E)=\varnothing$; moreover, $A \cap \operatorname{cl}(Y)=\varnothing$ by (2.18), hence

$$
\partial F \cap \partial B_{r} \subset \operatorname{cl}(F) \cap \partial B_{r} \subset \operatorname{cl}(E) \cup\left(\operatorname{cl}\left(N_{\eta}(Y)\right) \cap \partial B_{r} 0\right)=\operatorname{cl}(E) \cup \operatorname{cl}(Y),
$$

and we deduce 2.19). To prove 2.20): if $y \in \partial B_{r} \backslash \operatorname{cl}(E)$, then $y$ belongs to one of the open connected components of $\partial B_{r} \backslash \partial E$, so it is either $y \in A$, or $y \in \partial B_{r} \backslash \operatorname{cl}(A) \subset Y$. To prove (2.21): by (2.18) we have $A \cap \operatorname{cl}(Y)=\varnothing$, so that by $(2.20)$

$$
\operatorname{cl}(Y) \backslash Y \subset \partial B_{r} \backslash(A \cup Y) \subset \partial B_{r} \cap \operatorname{cl}(E),
$$

and we conclude by $\left(E \cap \partial B_{r}\right) \cap \operatorname{cl}(Y)=\varnothing$ (again, thanks to (2.18)). Finally, (2.22) and the inclusion " $\subset$ " in (2.23) are obvious, while the other inclusion in (2.23) follows from (2.12). Having proved the claim, we complete the proof. By definition, $F \subset \Omega$ is open. We show that $\Omega \cap \partial F$ is $\mathcal{C}$-spanning $W$. Given $\gamma \in \mathcal{C}$, if $\gamma \cap \partial E \backslash \operatorname{cl}\left(B_{r}\right) \neq \varnothing$, then $\gamma \cap \partial F \neq \varnothing$ by (2.2); if instead $\gamma \cap \partial E \backslash \operatorname{cl}\left(B_{r}\right)=\varnothing$, then necessarily $\gamma \cap \partial E \cap \operatorname{cl}\left(B_{r}\right) \neq \varnothing$. Now, if $\gamma \cap \partial E \cap \partial B_{r} \neq \varnothing$ then $\gamma \cap \partial F \neq \varnothing$ by (2.17); otherwise we actually have $\gamma \cap \partial E \backslash B_{r}=\varnothing$, and thus, by Lemma 2.2. $\gamma$ intersects two distinct connect components of $\partial B_{r} \backslash \partial E$, and at least one of them is contained in $\partial F \cap \partial B_{r}$ : indeed, $\partial F \cap \partial B_{r}$ contains $\partial B_{r} \backslash \mathrm{cl}(A)$ by 2.16, where $\operatorname{cl}(A)$ is disjoint from all the connected components of $\partial B_{r} \backslash \partial E$ that are different from $A$.

Now, we prove (2.3), (2.4), and (2.5). First notice that (2.16), (2.19), (2.22), and $\mathcal{H}^{n}\left(\partial B_{r} \cap \partial E\right)=0$ imply that

$$
\partial F \cap \partial B_{r}=\partial B_{r} \backslash A \text { modulo } \mathcal{H}^{n},
$$


which in turn implies 2.3). Next, we claim that

$$
\begin{aligned}
\mathcal{H}^{n}(\Omega \cap \partial F) \leq & \mathcal{H}^{n}\left(\Omega \cap \partial E \backslash B_{r}\right)+\mathcal{H}^{n}\left(E \cap \partial B_{r}\right) \\
& +(2+C(n) \eta) \mathcal{H}^{n}\left(\partial B_{r} \backslash\left(A \cup\left(E \cap \partial B_{r}\right)\right)\right) \\
& +C(n) \eta \mathcal{H}^{n-1}\left(\partial E \cap \partial B_{r}\right) .
\end{aligned}
$$

To prove the claim, first by $\mathcal{H}^{n}\left(\partial E \cap \partial B_{r}\right)=0$, 2.2), and 2.19), we have

$$
\begin{aligned}
\mathcal{H}^{n}(\Omega \cap \partial F) & =\mathcal{H}^{n}\left(\Omega \cap \partial E \backslash B_{r}\right)+\mathcal{H}^{n}\left(\operatorname{cl}\left(B_{r}\right) \cap \partial F\right) \\
& \leq \mathcal{H}^{n}\left(\Omega \cap \partial E \backslash B_{r}\right)+\mathcal{H}^{n}\left(\partial B_{r} \backslash A\right)+\mathcal{H}^{n}\left(B_{r} \cap \partial F\right) .
\end{aligned}
$$

If $g(y, t)=y-t v_{B_{r}}(y)$, then by 2.13)

$$
B_{r} \cap \partial F=B_{r} \cap \partial N_{\eta}(Y)=g(Y, \eta) \cup g((\operatorname{cl}(Y) \backslash Y) \times[0, \eta]),
$$

so that 2.21], the $\mathcal{H}^{n-1}$-rectifiability of $\partial E \cap \partial B_{r}$, and the area formula give us

(2.27) $\mathcal{H}^{n}\left(B_{r} \cap \partial F\right) \leq(1+C(n) \eta) \mathcal{H}^{n}(Y)+C(n) \eta \mathcal{H}^{n-1}\left(\partial E \cap \partial B_{r}\right)$.

By $\mathcal{H}^{n}\left(\partial E \cap \partial B_{r}\right)=0$, 2.22), and 2.23) we have

$$
\mathcal{H}^{n}(Y)=\mathcal{H}^{n}\left(\partial B_{r} \backslash\left(A \cup\left(E \cap \partial B_{r}\right)\right)\right),
$$

so that 2.26, 2.27), and 2.28) imply 2.25). Letting $\eta \rightarrow 0^{+}$in 2.25) we find (2.4), and doing the same in 2.27) and 2.28), we deduce 2.5.

Step 2. In the case $A \cap E=\varnothing$, we now allow for the set $S$ defined in (2.8) to be nonempty. In this case, if $F$ is defined as in 2.9) then the inclusion 2.17) is not true in general, and $\Omega \cap \partial F$ may fail to be $\mathcal{C}$-spanning $W$. We then modify the construction as detailed in Remark 2.6, defining $F$ as in 2.10). We notice that $F \subset \Omega$ is open, and that 2.2 holds true, since once again $F \backslash \operatorname{cl}\left(B_{r}\right)=E \backslash \operatorname{cl}\left(B_{r}\right)$. Moreover, we have

$$
\begin{aligned}
& B_{r} \cap \partial F=B_{r} \cap \partial N_{\eta}(Z), \\
& Z \subset \partial F \cap \partial B_{r}, \\
& E \cap \partial B_{r} \subset \partial F \cap \partial B_{r}, \\
& \partial B_{r} \backslash \operatorname{cl}(A) \subset \partial F \cap \partial B_{r}, \\
& \partial E \cap \partial B_{r} \subset \partial F \cap \partial B_{r}, \\
& A, E \cap \partial B_{r}, Y \text { are open and disjoint in } \partial B_{r}, \\
& \partial F \cap \partial B_{r} \subset\left[\partial B_{r} \backslash A\right] \cup\left[\partial B_{r} \cap\left\{\mathrm{d}_{S} \leq \eta\right\}\right], \\
& \partial B_{r} \backslash \operatorname{cl}(E) \subset A \cup Y, \\
& \operatorname{cl}(Y) \backslash Y \subset \partial B_{r} \cap \partial E, \\
& \operatorname{cl}(A) \backslash A \subset \partial B_{r} \cap \partial E, \\
& \operatorname{cl}\left(E \cap \partial B_{r}\right) \backslash\left(E \cap \partial B_{r}\right) \subset \partial B_{r} \cap \partial E .
\end{aligned}
$$

The proofs of 2.29), 2.30, 2.31), and 2.32) are identical to the proofs of the corresponding statements in Step 1 with $Z$ replacing $Y$; 2.33 then follows from 
(2.30), 2.31), and (2.32), since $S \subset U_{\eta}(S) \backslash \operatorname{cl}\left(E \cap \partial B_{r}\right) \subset Z$; 2.34) is obvious. To prove 2.35): as in Step 1, $A \cap \operatorname{cl}(E)=\varnothing$ and $A \cap \operatorname{cl}(Y)=\varnothing$ by (2.34), and

$$
\begin{aligned}
\partial F \cap \partial B_{r} & \subset \operatorname{cl}(F) \cap \partial B_{r} \subset \operatorname{cl}(E) \cup\left(\operatorname{cl}\left(N_{\eta}(Z)\right) \cap \partial B_{r}\right) \\
& \subset \operatorname{cl}(E) \cup \operatorname{cl}(Y) \cup \operatorname{cl}\left(U_{\eta}(S)\right),
\end{aligned}
$$

so that 2.35 follows from the fact that $\operatorname{cl}\left(U_{\eta}(S)\right) \subset \partial B_{r} \cap\left\{\mathrm{d}_{S} \leq \eta\right\}$. Next, we notice that (2.36), (2.37), 2.38), and (2.39) are shown analogously to Step 1 (with the identity in (2.23) which becomes an inclusion in (2.39) due to $S$ possibly being not empty). With the above at our disposal, we proceed now to verify the claims of the lemma. First, the proof that $\Omega \cap \partial F$ is $\mathcal{C}$-spanning $W$ follows verbatim the argument from Step 1. Next, 2.32, 2.35, 2.38), and $\mathcal{H}^{n}\left(\partial E \cap \partial B_{r}\right)=0$ imply that

$$
\mathcal{H}^{n}\left(\left(\partial F \cap \partial B_{r}\right) \Delta\left(\partial B_{r} \backslash A\right)\right) \leq \mathcal{H}^{n}\left(\partial B_{r} \cap\left\{\mathrm{d}_{S} \leq \eta\right\}\right) .
$$

In particular, since $\mathcal{H}^{n-1}(S)<\infty$, it holds that

$$
\lim _{\eta \rightarrow 0^{+}} \mathcal{H}^{n}\left(\left(\partial F \cap \partial B_{r}\right) \Delta\left(\partial B_{r} \backslash A\right)\right)=0,
$$

that is, (2.3). Next, we proceed with estimating $\mathcal{H}^{n}(\Omega \cap \partial F)$. We first notice that, by $(2.2)$ and $\mathcal{H}^{n}\left(\partial E \cap \partial B_{r}\right)=0$,

$$
\begin{aligned}
\mathcal{H}^{n}(\Omega \cap \partial F) & =\mathcal{H}^{n}\left(\Omega \cap \partial E \backslash B_{r}\right)+\mathcal{H}^{n}\left(\operatorname{cl}\left(B_{r}\right) \cap \partial F\right) \\
& \leq \mathcal{H}^{n}\left(\Omega \cap \partial E \backslash B_{r}\right)+\mathcal{H}^{n}\left(\partial F \cap \partial B_{r}\right)+\mathcal{H}^{n}\left(B_{r} \cap \partial F\right) .
\end{aligned}
$$

Setting, as in Step 1, $g(y, t)=y-t v_{B_{r}}(y)$, we then have from 2.29) that

$$
B_{r} \cap \partial F=B_{r} \cap \partial N_{\eta}(Z)=g(Z, \eta) \cup g((\operatorname{cl}(Z) \backslash Z) \times[0, \eta]) .
$$

By the area formula, we can easily estimate

$$
\begin{aligned}
\mathcal{H}^{n} & (g(Z, \eta)) \\
& \leq(1+C(n) \eta) \mathcal{H}^{n}(Z) \\
& \leq(1+C(n) \eta)\left(\mathcal{H}^{n}(Y)+\mathcal{H}^{n}\left(\partial B_{r} \cap\left\{\mathrm{d}_{S}<\eta\right\}\right)\right) \\
& \leq(1+C(n) \eta)\left(\mathcal{H}^{n}\left(\partial B_{r} \backslash\left(A \cup\left(E \cap \partial B_{r}\right)\right)\right)+\mathcal{H}^{n}\left(\partial B_{r} \cap\left\{\mathrm{d}_{S}<\eta\right\}\right)\right) .
\end{aligned}
$$

On the other hand, it holds that

$$
\operatorname{cl}(Z) \backslash Z \subset[\operatorname{cl}(Y) \backslash(Y)] \cup[\operatorname{cl}(\widehat{U}) \backslash \hat{U}],
$$

where $\widehat{U}=U_{\eta}(S) \backslash \operatorname{cl}\left(E \cap \partial B_{r}\right)$. Since $\operatorname{cl}(\widehat{U}) \subset \operatorname{cl}\left(U_{\eta}(S)\right) \backslash\left(E \cap \partial B_{r}\right), 2.39$ ) implies that

$$
\operatorname{cl}(\hat{U}) \backslash \widehat{U} \subset\left(\partial B_{r} \cap\left\{\mathrm{d}_{S}=\eta\right\}\right) \cup\left(\partial B_{r} \cap \partial E\right),
$$

and thus 2.37) yields

$$
\begin{aligned}
& \mathcal{H}^{n}(g((\operatorname{cl}(Z) \backslash Z) \times[0, \eta])) \\
& \quad \leq C(n) \eta\left(\mathcal{H}^{n-1}\left(\partial B_{r} \cap \partial E\right)+\mathcal{H}^{n-1}\left(\partial B_{r} \cap\left\{\mathrm{d}_{S}=\eta\right\}\right)\right) .
\end{aligned}
$$


D. KING, F. MAGGI, AND S. STUVARD

By applying the coarea formula to $\mathrm{d}_{S}$, it holds for every $0<\sigma<r / 2$ that

$$
\int_{0}^{\sigma} \mathcal{H}^{n-1}\left(\partial B_{r} \cap\left\{\mathrm{d}_{S}=\eta\right\}\right) d \eta=\mathcal{H}^{n}\left(\partial B_{r} \cap\left\{\mathrm{d}_{S} \leq \sigma\right\}\right)<\infty
$$

and thus there exists a decreasing sequence $\left\{\eta_{k}\right\}_{k=1}^{\infty}$ with $\lim _{k \rightarrow \infty} \eta_{k}=0$ such that $\partial B_{r} \cap\left\{\mathrm{d}_{S}=\eta_{k}\right\}$ is $\mathcal{H}^{n-1}$-rectifiable and

$$
\lim _{k \rightarrow \infty} \eta_{k} \mathcal{H}^{n-1}\left(\partial B_{r} \cap\left\{\mathrm{d}_{S}=\eta_{k}\right\}\right)=0 .
$$

If $F_{k}$ is the sequence of cup competitors defined by 2.10$)$ in correspondence with the choice $\eta=\eta_{k}$, we then have from (2.43), (2.45), 2.37), and (2.46) that $\Omega \cap \partial F_{k}$ is $\mathcal{H}^{n}$-rectifiable, and from (2.42), (2.41), (2.44), (2.47), and (2.49) that

$$
\begin{aligned}
& \limsup _{k \rightarrow \infty} \mathcal{H}^{n}\left(B_{r} \cap \partial F_{k}\right) \leq \mathcal{H}^{n}\left(\partial B_{r} \backslash\left(A \cup\left(E \cap \partial B_{r}\right)\right)\right), \\
& \limsup _{k \rightarrow \infty} \mathcal{H}^{n}\left(\Omega \cap \partial F_{k}\right) \leq \mathcal{H}^{n}\left(\Omega \cap \partial E \backslash B_{r}\right)+2 \mathcal{H}^{n}\left(\partial B_{r} \backslash A\right) .
\end{aligned}
$$

Defining $F_{\eta}=F_{\eta_{k}}$ for all $\eta \in\left(\eta_{k+1}, \eta_{k}\right)$ then allows us to conclude both 2.4 and (2.5).

Step 3. We now assume that $A \subset E$, and define $F$ by (2.11), that is,

$$
F=\left(E \cup B_{r}\right) \backslash \operatorname{cl}\left(N_{\eta}(Y)\right), \quad Y=\left(E \cap \partial B_{r}\right) \backslash \operatorname{cl}(A) .
$$

We claim that (2.2) holds, as well as

$$
\begin{aligned}
Y & \subset \partial F \cap \partial B_{r}, \\
\partial B_{r} \backslash E & \subset \partial F \cap \partial B_{r}, \\
\partial B_{r} \backslash \operatorname{cl}(A) & \subset \partial F \cap \partial B_{r}, \\
B_{r} \cap \partial F & \subset B_{r} \cap \partial N_{\eta}(Y),
\end{aligned}
$$

$A, \partial B_{r} \backslash \operatorname{cl}(E), Y$ are open and disjoint in $\partial B_{r}$,

$$
\begin{aligned}
& \partial F \cap \partial B_{r} \subset \partial B_{r} \backslash A, \\
& \operatorname{cl}(A) \backslash A \subset \partial B_{r} \cap \partial E, \\
& \operatorname{cl}(Y) \backslash Y \subset \partial B_{r} \cap \partial E .
\end{aligned}
$$

First, $F \backslash \operatorname{cl}\left(B_{r}\right)=E \backslash \operatorname{cl}\left(B_{r}\right)$ implies 2.2). To prove 2.53): since $E$ is open we have $E \cap \partial B_{r} \subset \operatorname{cl}\left(E \backslash \operatorname{cl}\left(B_{r}\right)\right)=\operatorname{cl}\left(F \backslash \operatorname{cl}\left(B_{r}\right)\right)$ (by (2.52)), thus $Y \subset \operatorname{cl}(F)$; we conclude as $Y \cap F=\varnothing$. As $F \cap \partial B_{r} \subset E \cap \partial B_{r}$, to prove (2.54) we just need to show that $\partial B_{r} \backslash E \subset \operatorname{cl}(F)$ : since $\operatorname{cl}(U) \backslash \operatorname{cl}(V) \subset \operatorname{cl}(U \backslash \operatorname{cl}(V))$ for every $U, V \subset \mathbb{R}^{n+1}$, by $\partial B_{r} \cap \operatorname{cl}\left(N_{\eta}(Y)\right) \subset \operatorname{cl}(E)$ :

$$
\partial B_{r} \backslash \operatorname{cl}(E) \subset \operatorname{cl}\left(B_{r}\right) \backslash \operatorname{cl}\left(N_{\eta}(Y)\right) \subset \operatorname{cl}\left(B_{r} \backslash \operatorname{cl}\left(N_{\eta}(Y)\right) \subset \operatorname{cl}(F),\right.
$$$$
\left(\partial B_{r} \cap \partial E\right) \backslash \operatorname{cl}\left(N_{\eta}(Y)\right) \subset \operatorname{cl}(E) \backslash \operatorname{cl}\left(N_{\eta}(Y)\right) \subset \operatorname{cl}\left(E \backslash \operatorname{cl}\left(N_{\eta}(Y)\right)\right) \subset \operatorname{cl}(F),
$$$$
\partial B_{r} \cap \partial E \cap \operatorname{cl}\left(N_{\eta}(Y)\right) \subset \partial E \cap \operatorname{cl}(Y) \subset \partial F,
$$ 
where the last inclusion follows by (2.53). Next, 2.55) follows by 2.53, 2.54 , and

$$
\partial B_{r} \backslash \operatorname{cl}(A)=\left[\left(E \cap \partial B_{r}\right) \backslash \operatorname{cl}(A)\right] \cup\left[\partial B_{r} \backslash(E \cup \operatorname{cl}(A))\right] \subset Y \cup\left(\partial B_{r} \backslash E\right) .
$$

To prove (2.56): setting $V^{c}=\mathbb{R}^{n+1} \backslash V$, by $B_{r} \cap F=B_{r} \cap \operatorname{cl}\left(N_{\eta}(Y)\right)^{c}$ we find $B_{r} \cap \partial F=B_{r} \cap \partial\left[\operatorname{cl}\left(N_{\eta}(Y)\right)^{c}\right]$, where, as a general fact on open set $U \subset \mathbb{R}^{n+1}$, we have

$$
\partial\left[\operatorname{cl}(U)^{c}\right]=\operatorname{cl}\left(\operatorname{cl}(U)^{c}\right) \backslash \operatorname{cl}(U)^{c}=\operatorname{cl}(U) \cap \operatorname{cl}\left(\operatorname{cl}(U)^{c}\right), \quad \operatorname{cl}\left(\operatorname{cl}(U)^{c}\right) \subset U^{c},
$$

and thus $\partial\left[\operatorname{cl}(U)^{c}\right] \subset \partial U$. Next, 2.57) is obvious, and implies $A \cap \operatorname{cl}(Y)=\varnothing$ where $\operatorname{cl}(Y)=\operatorname{cl}\left(N_{\eta}(Y)\right) \cap \partial B_{r}$, so that $A \cap \partial B_{r} \subset E \cap \partial B_{r} \backslash \operatorname{cl}\left(N_{\eta}(Y)\right)=$ $F \cap \partial B_{r}$, and (2.58) follows. To prove (2.59), just notice that $A \subset E$ and $A$ is a connected component of $\partial B_{r} \backslash \partial E$. To prove (2.60): trivially, $\operatorname{cl}(Y) \backslash Y \subset \operatorname{cl}(Y) \subset$ $\partial B_{r} \cap \operatorname{cl}(E)$, while by definition of $Y$ and by $\operatorname{cl}(Y) \cap A=\varnothing$

$$
\begin{aligned}
E \cap(\operatorname{cl}(Y) \backslash Y) & =\left(\operatorname{cl}(Y) \cap\left(E \cap \partial B_{r}\right)\right) \backslash Y=\operatorname{cl}(Y) \cap\left(E \cap \partial B_{r}\right) \cap \operatorname{cl}(A) \\
& =\left(E \cap \partial B_{r}\right) \cap \operatorname{cl}(Y) \cap \partial A \subset E \cap(\operatorname{cl}(A) \backslash A)=\varnothing,
\end{aligned}
$$

thanks to 2.59). We have completed the claim. Next, by (2.55), 2.58), 2.59), and by $\mathcal{H}^{n}\left(\partial B_{r} \cap \partial E\right)=0$, we deduce (2.24) and thus (2.3), while $\Omega \cap \partial F$ is $\mathcal{C}$-spanning $W$ thanks to 2.2), Lemma 2.2, 2.55), and 2.54). Finally,

$$
\mathcal{H}^{n}(\Omega \cap \partial F) \leq \mathcal{H}^{n}\left(\partial E \backslash B_{r}\right)+\mathcal{H}^{n}\left(\partial B_{r} \backslash E\right)
$$

$$
+(2+C(n) \eta) \mathcal{H}^{n}\left(E \cap \partial B_{r} \backslash A\right)+C(n) \eta \mathcal{H}^{n-1}\left(\partial E \cap \partial B_{r}\right) .
$$

Indeed, by $\mathcal{H}^{n}\left(\partial E \cap \partial B_{r}\right)=0,2.2$, and 2.58),

$$
\begin{aligned}
\mathcal{H}^{n}(\Omega \cap \partial F) \leq & \mathcal{H}^{n}\left(\partial E \backslash B_{r}\right)+\mathcal{H}^{n}\left(\partial F \cap \operatorname{cl}\left(B_{r}\right)\right) \\
\leq & \mathcal{H}^{n}\left(\partial E \backslash B_{r}\right)+\mathcal{H}^{n}\left(\partial B_{r} \backslash A\right)+\mathcal{H}^{n}\left(B_{r} \cap \partial F\right) \\
\leq & \mathcal{H}^{n}\left(\partial E \backslash B_{r}\right)+\mathcal{H}^{n}\left(\partial B_{r} \backslash E\right) \\
& +\mathcal{H}^{n}\left(\left(E \cap \partial B_{r}\right) \backslash A\right)+\mathcal{H}^{n}\left(B_{r} \cap \partial F\right) ;
\end{aligned}
$$

by (2.56), 2.60), the $\mathcal{H}^{n-1}$-rectifiability of $\partial E \cap \partial B_{r}$, and the area formula

$$
\begin{aligned}
\mathcal{H}^{n}\left(B_{r} \cap \partial F\right) & \leq \mathcal{H}^{n}\left(B_{r} \cap \partial N_{\eta}(Y)\right) \\
& \leq(1+C(n) \eta) \mathcal{H}^{n}(Y)+C(n) \eta \mathcal{H}^{n-1}\left(\partial E \cap \partial B_{r}\right),
\end{aligned}
$$

while (2.59) and $\mathcal{H}^{n}\left(\partial B_{r} \cap \partial E\right)=0$ give

$$
\mathcal{H}^{n}(Y)=\mathcal{H}^{n}\left(\left(E \cap \partial B_{r}\right) \backslash \operatorname{cl}(A)\right)=\mathcal{H}^{n}\left(\left(E \cap \partial B_{r}\right) \backslash A\right) .
$$

We thus deduce (2.61). As $\eta \rightarrow 0^{+}$in (2.61) and in (2.63) we get (2.4) and (2.6).

In the following lemma we introduce the notion of exterior cup competitor. We set

$$
M_{\eta}(Y)=\left\{y+t v_{B}(y): y \in Y, t \in(0, \eta)\right\}, \quad \eta>0,
$$

whenever $B$ is an open ball and $Y \subset \partial B$. 

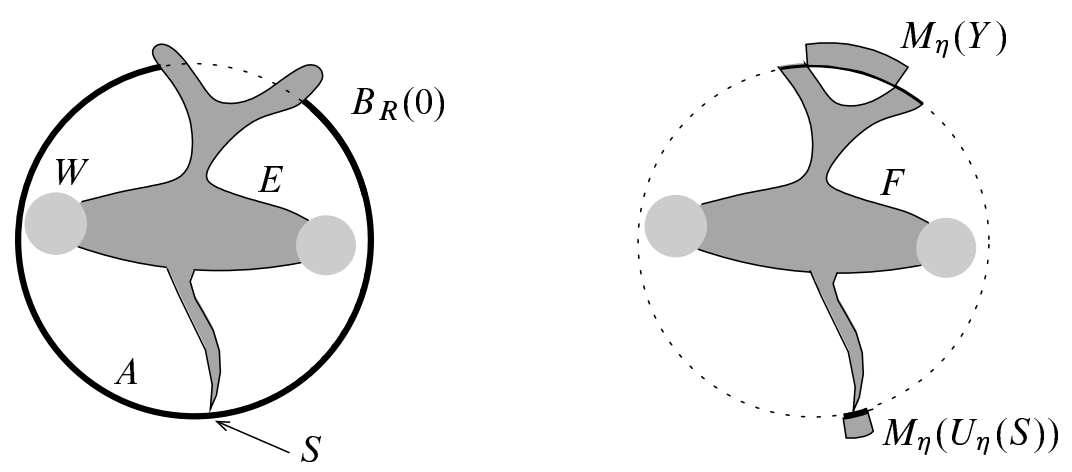

FIGURE 2.2. An exterior cup competitor. Notice that, for $S$ to be nonempty and nondisconnecting $A$, it must be $n \geq 2$.

LEMMA 2.7 (Exterior cup competitor). Let $E \in \mathcal{E}$ be such that $\Omega \cap \partial E$ is $\mathcal{C}$-spanning $W$, let $R>0$ be such that $W \Subset B_{R}(0)$ and $\partial E \cap \partial B_{R}(0)$ is $\mathcal{H}^{n-1}$-rectifiable, and let $A$ be a connected component of $\partial B_{R}(0) \backslash \partial E$ such that $A \cap E=\varnothing$. For every $\eta \in(0,1)$ there exists a set $F=F_{\eta} \in \mathcal{E}$ such that $\Omega \cap \partial F$ is $\mathcal{C}$-spanning $W$ and

$$
\limsup _{\eta \rightarrow 0^{+}} \mathcal{H}^{n}(\Omega \cap \partial F) \leq \mathcal{H}^{n}\left(\Omega \cap B_{R}(0) \cap \partial E\right)+2 \mathcal{H}^{n}\left(\partial B_{R}(0) \backslash A\right) .
$$

PROOF. The proof consists of a minor modification of Step 1 and Step 2 in the proof of Lemma 2.5. Precisely, the exterior cup competitor defined by $E$ and $A$ is given by

$$
F=\left(E \cap B_{R}(0)\right) \cup M_{\eta}(Z),
$$

where

$$
\begin{aligned}
Z & =Y \cup\left(U_{\eta}(S) \backslash \operatorname{cl}\left(E \cap \partial B_{R}(0)\right)\right), \\
Y & =\partial B_{R}(0) \backslash\left(\operatorname{cl}\left(E \cap \partial B_{R}(0)\right) \cup \operatorname{cl}(A)\right), \\
U_{\eta}(S) & =\partial B_{R}(0) \cap\left\{\mathrm{d}_{S}<\eta\right\}, \\
S & =\partial E \cap \operatorname{cl}(A) \backslash\left(\operatorname{cl}\left(E \cap \partial B_{R}(0)\right) \cup \operatorname{cl}(Y)\right) ;
\end{aligned}
$$

see Figure 2.2. If $\gamma \in \mathcal{C}$ is such that $\gamma \cap \partial E \cap \operatorname{cl}\left(B_{R}(0)\right)=\varnothing$, then an adaptation of Step 1 in the proof of Lemma 2.4 shows that there exists a connected component of $\gamma \backslash B_{R}(0)$ which is diffeomorphic to an interval, and whose endpoints belong to distinct connected components of $\left(\mathbb{R}^{n+1} \backslash B_{R}(0)\right) \backslash \partial E$. Using this fact, and since $\partial F \cap B_{R}(0)=\partial E \cap B_{R}(0)$, we just need to show that $\partial B_{R}(0) \cap \partial F$ contains $\partial B_{R}(0) \cap \partial E$ as well as $\partial B_{R}(0) \backslash \operatorname{cl}(A)$ in order to show that $\Omega \cap \partial F$ is $\mathcal{C}$-spanning $W$. This is done by repeating with minor variations the considerations contained in step two of the proof of Lemma 2.5. The proof of 2.64) is obtained in a similar way, and the details are omitted. 


\subsection{Slab competitors}

Bi-Lipschitz deformations of cup competitors can be used to generate new competitors thanks to Lemma 2.3. We will crucially use this remark to replace balls with "slabs" (see Figures 3.2, 3.3, and 3.4) and obtain sharp area concentration estimates in step five of the proof of Theorem 1.4 as well as in the proof of Theorem 1.6, see, e.g., 4.7). Given $\tau \in(0,1), x \in \mathbb{R}^{n+1}, r>0$, and $v \in \mathbb{S}^{n}$, we set

$$
S_{\tau, r}^{v}(x)=\left\{y \in B_{r}(x):|(y-x) \cdot v|<\tau r\right\},
$$

and we claim the existence of a bi-Lipschitz map $\Phi: \mathbb{R}^{n+1} \rightarrow \mathbb{R}^{n+1}$ with

$$
\begin{gathered}
\{\Phi \neq \mathrm{id}\} \Subset B_{2 r}(x), \quad \Phi\left(B_{2 r}(x)\right)=B_{2 r}(x), \\
\Phi\left(\partial S_{\tau, t}^{v}(x)\right)=\partial B_{t}(x) \quad \forall t \in(0, r),
\end{gathered}
$$

and such that Lip $\Phi$ and Lip $\Phi^{-1}$ depend only on $n$ and $\tau$. Indeed, assuming without loss of generality that $x=0$, there is a convex, degree-1 positively homogeneous function $\varphi: \mathbb{R}^{n+1} \rightarrow[0, \infty)$ such that $S_{\tau, t}^{\nu}(0)=\{\varphi<t\}$ for every $t>0$. Taking $\eta_{r}:[0, \infty) \rightarrow[0, \infty)$ smooth, decreasing, and such that $\eta=1$ on $[0,4 r / 3]$ and $\eta=0$ on $[5 r / 3, \infty)$, we set

$$
\Phi(x)=\eta_{r}(|x|) \frac{\varphi(x)}{|x|} x+\left(1-\eta_{r}(|x|)\right) x .
$$

Noticing that $\Phi$ is a smooth interpolation between linear maps on each half-line $\{t x: t \geq 0\}$, and observing that the slopes of these linear maps change in a Lipschitz way with respect to the angular variable, one sees that $\Phi$ has the required properties.

LEMMA 2.8 (Slab competitors). Let $E \in \mathcal{E}$ be such that $\Omega \cap \partial E$ is $\mathcal{C}$-spanning $W$, and let $B_{2 r}(x) \Subset \Omega, v \in \mathbb{S}^{n}, \tau \in(0,1)$ with $\partial S_{\tau, r}^{v}(x) \cap \partial E \mathcal{H}^{n-1}$-rectifiable. Let $A$ be an open connected component of $\partial S_{\tau, r}^{\nu}(x) \backslash \partial E$. Then for every $\eta \in(0, r / 2)$, there exists $F \in \mathcal{E}$ such that $\Omega \cap \partial F$ is $\mathcal{C}$-spanning $W$,

$$
\begin{gathered}
F \backslash \operatorname{cl}\left(S_{\tau, r}^{v}(x)\right)=E \backslash \operatorname{cl}\left(S_{\tau, r}^{v}(x)\right), \\
\lim _{\eta \rightarrow 0^{+}} \mathcal{H}^{n}\left(\left(\partial F \cap \partial S_{\tau, r}^{v}(x)\right) \Delta\left(\partial S_{\tau, r}^{v}(x) \backslash A\right)\right)=0,
\end{gathered}
$$

and such that if $A \cap E=\varnothing$, then

$$
\limsup _{\eta \rightarrow 0^{+}} \mathcal{H}^{n}\left(S_{\tau, r}^{\nu}(x) \cap \partial F\right) \leq C(n, \tau) \mathcal{H}^{n}\left(\partial S_{\tau, r}^{v}(x) \backslash(A \cup E)\right) ;
$$

while, if $A \subset E$, then

$$
\limsup _{\eta \rightarrow 0^{+}} \mathcal{H}^{n}\left(S_{\tau, r}^{v}(x) \cap \partial F\right) \leq C(n, \tau) \mathcal{H}^{n}\left(E \cap \partial S_{\tau, r}^{v}(x) \backslash A\right) .
$$

PROOF. Let us set for brevity $S_{r}=S_{\tau, r}^{v}(x)$ and $B_{r}=B_{r}(x)$. By Lemma 2.3. $\Phi(E) \in \mathcal{E}$ and $\Omega \cap \partial \Phi(E)$ is $\mathcal{C}$-spanning $W$. Since $\Phi$ is an homeomorphism between $\partial S_{r}$ and $\partial B_{r}, \Phi(A)$ is an open connected component of $\partial B_{r} \backslash \partial \Phi(E)$. Depending on whether $A \cap E=\varnothing$ or $A \subset E$, and thus, respectively, depending 
on whether $\Phi(A) \cap \Phi(E)=\varnothing$ or $\Phi(A) \cap \Phi(E) \neq \varnothing$, we consider the cup competitor $G$ defined by $\Phi(E)$ and $\Phi(A)$, so that

$$
G=\left(\Phi(E) \backslash \operatorname{cl}\left(B_{r}\right)\right) \cup N_{\eta}(Z), \quad Z=Y \cup\left(U_{\eta}(S) \backslash \operatorname{cl}\left(\Phi(E) \cap \partial B_{r}\right)\right),
$$

where

$$
Y=\partial B_{r} \backslash\left(\operatorname{cl}\left(\Phi(E) \cap \partial B_{r}\right) \cup \operatorname{cl}(\Phi(A))\right), \quad U_{\eta}(S)=\partial B_{r} \cap\left\{\mathrm{d}_{S}<\eta\right\},
$$

with

$$
S=\partial \Phi(E) \cap \operatorname{cl}(\Phi(A)) \backslash\left[\operatorname{cl}\left(\Phi(E) \cap \partial B_{r}\right) \cup \operatorname{cl}(Y)\right],
$$

if $A \cap E=\varnothing$, see $(2.10)$, and

$$
G=\left(\Phi(E) \cup B_{r}\right) \backslash \operatorname{cl}\left(N_{\eta}(Y)\right), \quad Y=\left(\Phi(E) \cap \partial B_{r}\right) \backslash \operatorname{cl}(\Phi(A)),
$$

if $A \subset E$, see (2.11). Finally, we set $F=\Phi^{-1}(G)$. Since $G \in \mathcal{E}$ and $\Omega \cap \partial G$ is $\mathcal{C}$ spanning $W$, by Lemma 2.3 we find that $F \in \mathcal{E}$ and that $\Omega \cap \partial F$ is $\mathcal{C}$-spanning $W$. By construction $G \backslash \operatorname{cl}\left(B_{r}\right)=\Phi(E) \backslash \operatorname{cl}\left(B_{r}\right)$, so that 2.66) follows by

$$
F \backslash \operatorname{cl}\left(S_{r}\right)=\Phi^{-1}\left(G \backslash \operatorname{cl}\left(B_{r}\right)\right)=\Phi^{-1}\left(\Phi(E) \backslash \operatorname{cl}\left(B_{r}\right)\right)=E \backslash \operatorname{cl}\left(S_{r}\right) .
$$

By (2.3), $\mathcal{H}^{n}\left(\left(\partial B_{r} \cap \partial G\right) \Delta\left(\partial B_{r} \backslash \Phi(A)\right)\right) \rightarrow 0$ as $\eta \rightarrow 0^{+}$, which gives (2.67) by the area formula. Finally, 2.68) and (2.69) are deduced by the area formula, (2.5) and 2.6.

\subsection{Cone competitors}

As is customary in the analysis of area minimization problems, we want to compare $\mathcal{H}^{n}\left(B_{r}(x) \cap \partial E\right)$ with $\mathcal{H}^{n}\left(B_{r}(x) \cap \partial F\right)$, where $F$ is the cone spanned by $E \cap \partial B_{r}(x)$ over $x$,

$$
F=\left(E \backslash \operatorname{cl}\left(B_{r}(x)\right)\right) \cup\left\{(1-t) x+t y: y \in E \cap \partial B_{r}(x) t \in(0,1]\right\} .
$$

Following the terminology of [13], given $K \in \mathcal{S}$, the cone competitor $K^{\prime}$ of $K$ in $B_{r}(x)$ is similarly defined as

$$
K^{\prime}=\left(K \backslash B_{r}(x)\right) \cup\left\{(1-t) x+t y: y \in K \cap \partial B_{r}(x) t \in[0,1]\right\}
$$

and is indeed $\mathcal{C}$-spanning $W$ (since $K$ was). However, for some values of $r, \partial F \cap$ $B_{r}(x)$ may be strictly smaller than the cone competitor $K^{\prime}$ defined by the choice $K=\Omega \cap \partial E$ in $B_{r}(x)$, and thus it may fail to be $\mathcal{C}$-spanning; see Figure 2.3. By Sard's lemma, if $E$ has smooth boundary in $\Omega$, this issue can be avoided as, for a.e. $r, \partial E$, and $\partial B_{r}$ intersect transversally, and thus $\partial E \cap \partial B_{r}(x)$ is the boundary of $E \cap \partial B_{r}(x)$ relative to $\partial B_{r}(x)$; but working with smooth boundary leads to other difficulties when constructing cup competitors. We thus approximate $F$ (as defined in (2.70) ) in energy by means of diffeomorphic images of $E$.

Lemma 2.9 (Cone competitors). Let $E \in \mathcal{E}$ be such that $\Omega \cap \partial E$ is $\mathcal{C}$-spanning $W$, and let $B=B_{r}(x) \Subset \Omega$ be such that $E \cap \partial B_{r}(x)$ is $\mathcal{H}^{n}$-rectifiable, $\partial E \cap \partial B_{r}(x)$ is $\mathcal{H}^{n-1}$-rectifiable, and $r$ is a Lebesgue point of the maps $t \mapsto \mathcal{H}^{n}\left(E \cap \partial B_{t}(x)\right)$ 

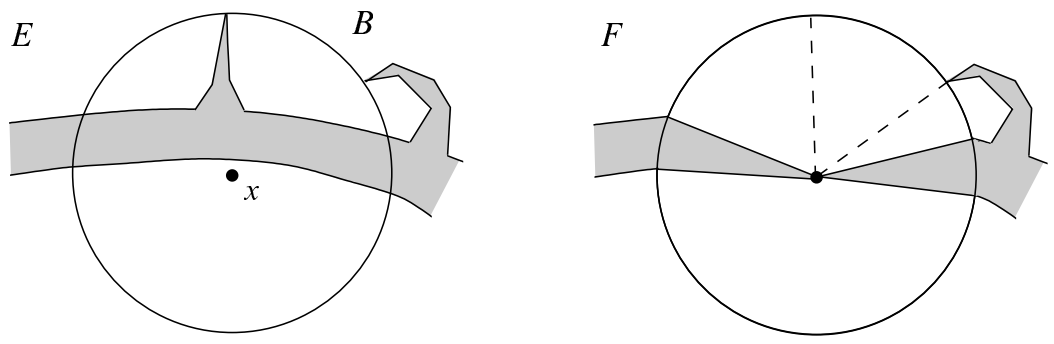

FIGURE 2.3. In this picture, the cone competitor $F$ defined by $E \cap \partial B_{r}$ as in 2.70 may fail to be $\mathcal{C}$-spanning $W$. Notice that the dashed lines are part of the cone competitor $K^{\prime}$ defined by $K=\Omega \cap \partial E$ in $B_{r}(x)$, which is indeed strictly larger than $\Omega \cap \partial F$.

and $t \mapsto \mathcal{H}^{n-1}\left(\partial E \cap \partial B_{t}(x)\right)$. Then for each $\eta \in(0, r / 2)$ there exists $F \in \mathcal{E}$ such that $F \Delta E \subset B_{r}(x), \Omega \cap \partial F$ is $\mathcal{C}$-spanning $W$, and

$$
\begin{gathered}
\limsup _{\eta \rightarrow 0^{+}} \mathcal{H}^{n}(\Omega \cap \partial F) \leq \mathcal{H}^{n}\left(\partial E \backslash B_{r}(x)\right)+\frac{r}{n} \mathcal{H}^{n-1}\left(\partial E \cap \partial B_{r}(x)\right), \\
\liminf _{\eta \rightarrow 0^{+}}|F| \geq\left|E \backslash B_{r}(x)\right|+\frac{r}{n+1} \mathcal{H}^{n}\left(E \cap \partial B_{r}(x)\right) .
\end{gathered}
$$

Proof. Let $x=0, r=1, B_{r}=B_{r}(0)$, and define a bi-Lipschitz map $f_{\eta}$ by $f_{\eta}(0)=0$ and $f_{\eta}(x)=u_{\eta}(|x|) \hat{x}$ if $x \neq 0$, where $\hat{x}=x /|x|$ and $u_{\eta}: \mathbb{R} \rightarrow$ $[0, \infty)$ is given by

$$
u_{\eta}(t):= \begin{cases}\max \{0, \eta t\} & \text { for } t \leq 1-\eta, \\ \eta(1-\eta)+\frac{t-(1-\eta)}{\eta}(1-\eta(1-\eta)) & \text { for } t \in[1-\eta, 1] \\ t & \text { for } t \geq 1\end{cases}
$$

so that $u_{\eta}(t) \leq t$ for $t \geq 0$. Clearly, $\left\{f_{\eta} \neq\right.$ id $\} \subset B_{1}$ and $f_{\eta}\left(B_{1}\right) \subset B_{1}$. The open set $F=f_{\eta}(E)$ is such that $\Omega \cap \partial F=f_{\eta}(\Omega \cap \partial E)$, so that $\Omega \cap \partial F$ is $\mathcal{H}^{n}$ rectifiable and, by Lemma 2.3, $\mathcal{C}$-spanning $W$. Thanks to the area formula, 2.71 will follow by showing

$$
\limsup _{\eta \rightarrow 0^{+}} \int_{B_{1} \cap \partial E} J^{\partial E} f_{\eta} d \mathcal{H}^{n} \leq \frac{1}{n} \mathcal{H}^{n-1}\left(\partial E \cap \partial B_{1}\right)
$$

Trivially, the integral over $B_{1-\eta} \cap \partial E$ is bounded by $C(n) \eta^{n} \mathcal{H}^{n}(\Omega \cap \partial E)$. The integral over $B_{1} \backslash B_{1-\eta}$ is treated as in [13, step two, theorem 7]; by the coarea 
formula,

$$
\begin{aligned}
& \int_{\left(B_{1} \backslash B_{1-\eta}\right) \cap \partial E} J^{\partial E} f_{\eta} d \mathcal{H}^{n} \\
& =\int_{1-\eta}^{1} d t \int_{\partial B_{t} \cap \partial E \cap\left\{\left|v_{E} \cdot \hat{x}\right|<1\right\}} \frac{J^{\partial E} f_{\eta}}{\sqrt{1-\left(v_{E} \cdot \hat{x}\right)^{2}}} d \mathcal{H}^{n-1} \\
& \quad+\int_{\left(B_{1} \backslash B_{1-\eta}\right) \cap \partial E \cap\left\{\left|v_{E} \cdot \hat{x}\right|=1\right\}} J^{\partial E} f_{\eta} d \mathcal{H}^{n}
\end{aligned}
$$

where $v_{E}(x) \in T_{x}(\partial E) \cap \mathbb{S}^{n}$ at $\mathcal{H}^{n}$-a.e. $x \in \partial E$. By

$$
\nabla f_{\eta}(x)=\frac{u_{\eta}(|x|)}{|x|} \mathrm{Id}+\left(u_{\eta}^{\prime}(|x|)-\frac{u_{\eta}(|x|)}{|x|}\right) \widehat{x} \otimes \widehat{x},
$$

if $\left|v_{E}(x) \cdot \hat{x}\right|=1$, then $J^{\partial E} f_{\eta}=\left(u_{\eta}(|x|) /|x|\right)^{n} \leq 1$. Since

$$
\lim _{\eta \rightarrow 0^{+}} \mathcal{H}^{n}\left(\partial E \cap\left(B_{1} \backslash B_{1-\eta}\right)\right)=0,
$$

the second term on the right-hand side of 2.75) converges to 0 as $\eta \rightarrow 0^{+}$. As for the first term, by 2.76, we have, as explained later on,

$$
J^{\partial E} f_{\eta}(x) \leq 1+\sqrt{1-\left(v_{E}(x) \cdot \hat{x}\right)^{2}} u_{\eta}^{\prime}(|x|)\left(\frac{u_{\eta}(|x|)}{|x|}\right)^{n-1}
$$

for $\mathcal{H}^{n}$-a.e. $x \in \partial E$. The term corresponding to 1 in $(2.78)$ converges to 0 as $\eta \rightarrow 0^{+}$by (2.77). At the same time,

$$
\limsup _{\eta \rightarrow 0^{+}}\left|\int_{1-\eta}^{1}\left(\mathcal{H}^{n-1}\left(\partial E \cap \partial B_{t}\right)-\mathcal{H}^{n-1}\left(\partial E \cap \partial B_{1}\right)\right) u_{\eta}^{\prime}\left(\frac{u_{\eta}}{t}\right)^{n-1} d t\right|=0
$$

since $t=1$ is a Lebesgue point of $t \mapsto \mathcal{H}^{n-1}\left(\partial B_{t} \cap \partial E\right)$, and since $u_{\eta}^{\prime}(t) \leq 1 / \eta$ and $\left(u_{\eta}(t) / t\right) \leq 1$ for $t \geq 0$. Finally,

$$
\begin{aligned}
\int_{1-\eta}^{1} u_{\eta}^{\prime}\left(\frac{u_{\eta}}{t}\right)^{n-1} d t & \leq \frac{1}{(1-\eta)^{n-1}} \frac{u_{\eta}(1)^{n}-u_{\eta}(1-\eta)^{n}}{n} \\
& =\frac{1}{(1-\eta)^{n-1}} \frac{1-\eta^{n}(1-\eta)^{n}}{n} \rightarrow \frac{1}{n}
\end{aligned}
$$

as $\eta \rightarrow 0^{+}$, thus completing the proof of 2.71. The proof of 2.72) follows an analogous argument. The goal is to show that

$$
\liminf _{\eta \rightarrow 0^{+}} \int_{E \cap B_{1}} J f_{\eta} d x \geq \frac{1}{n+1} \mathcal{H}^{n}\left(E \cap \partial B_{1}\right),
$$

and by the coarea formula and 2.76 it is immediate to see that

$$
\int_{E \cap B_{1}} J f_{\eta} d x \geq \int_{1-\eta}^{1} u_{\eta}^{\prime}(t)\left(\frac{u_{\eta}(t)}{t}\right)^{n} \mathcal{H}^{n}\left(E \cap \partial B_{t}\right) d t .
$$


The estimate in 2.79) then readily follows using that $t=1$ is a Lebesgue point for the map $t \mapsto \mathcal{H}^{n}\left(E \cap \partial B_{t}\right)$, together with

$$
\int_{1-\eta}^{1} u_{\eta}^{\prime}(t)\left(\frac{u_{\eta}(t)}{t}\right)^{n} d t \geq \frac{1-\eta^{n+1}(1-\eta)^{n+1}}{n+1} \rightarrow \frac{1}{n+1} \quad \text { as } \eta \rightarrow 0^{+} .
$$

We finally explain how to deduce 2.78 from 2.76. For $x \in \partial^{*} E$, let $\left\{\tau_{i}\right\}_{i=1}^{n}$ be an orthonormal basis of $T_{x} \partial^{*} E$ such that $\left\{\tau_{i}\right\}_{i=1}^{n-1} \subset x^{\perp}$. In this way, we can take

and therefore compute by 2.76 that

$$
\tau_{n}=\frac{\hat{x}-\left(\hat{x} \cdot v_{E}(x)\right) v_{E}(x)}{\sqrt{1-\left(\hat{x} \cdot v_{E}(x)\right)^{2}}}
$$

$$
\begin{aligned}
& \nabla^{\partial E} f_{\eta}(x)\left[\tau_{i}\right]=\frac{u_{\eta}(|x|)}{|x|} \tau_{i} \quad \forall i=1, \ldots, n-1, \\
& \nabla^{\partial E} f_{\eta}(x)\left[\tau_{n}\right]=u_{\eta}^{\prime}(|x|) \sqrt{1-\left(\hat{x} \cdot v_{E}\right)^{2}} \hat{x}-\frac{u_{\eta}(|x|)}{|x|}\left(\hat{x} \cdot v_{E}\right) \frac{v_{E}-\left(\hat{x} \cdot v_{E}\right) \hat{x}}{\sqrt{1-\left(\hat{x} \cdot v_{E}\right)^{2}}},
\end{aligned}
$$

where we have set for brevity $v_{E}$ in place of $v_{E}(x)$. Therefore

$$
\begin{aligned}
J^{\partial E} f(x)^{2}= & \left|\bigwedge_{i=1}^{n} \nabla^{\partial E} f_{\eta}(x)\left[\tau_{i}\right]\right|^{2} \\
= & \left(\frac{u_{\eta}(|x|)}{|x|}\right)^{2 n}\left(\hat{x} \cdot v_{E}\right)^{2}\left|\tau_{1} \wedge \cdots \wedge \tau_{n-1} \wedge\left(\frac{v_{E}-\left(\hat{x} \cdot v_{E}\right) \hat{x}}{\sqrt{1-\left(\hat{x} \cdot v_{E}\right)^{2}}}\right)\right|^{2} \\
& +\left(\frac{u_{\eta}(|x|)}{|x|}\right)^{2(n-1)} u_{\eta}^{\prime}(|x|)^{2}\left(1-\left(\hat{x} \cdot v_{E}\right)^{2}\right)\left|\tau_{1} \wedge \cdots \wedge \tau_{n-1} \wedge \hat{x}\right|^{2} \\
\leq & 1+\left(\frac{u_{\eta}(|x|)}{|x|}\right)^{2(n-1)} u_{\eta}^{\prime}(|x|)^{2}\left(1-\left(\hat{x} \cdot v_{E}\right)^{2}\right),
\end{aligned}
$$

from which 2.78 follows thanks to $\sqrt{1+a} \leq 1+\sqrt{a}$ for $a \geq 0$.

\subsection{Nucleation lemma}

The following nucleation lemma can be found, with slightly different statements, in [1, VI(13)] or in [42, lemma 29.10].

LEMMA 2.10. Let $\xi(n)$ be the constant of Besicovitch's covering theorem in $\mathbb{R}^{n+1}$. If $T$ is closed, $A=\mathbb{R}^{n+1} \backslash T, 0<|E|<\infty, P(E ; A)<\infty, \tau>0$, and

$$
\sigma=\min \left\{\frac{\left|E \backslash I_{\tau}(T)\right|}{\tau P(E ; A)}, \frac{\xi(n)}{n+1}\right\}>0,
$$

then there exists $x \in E^{(1)} \backslash I_{\tau}(T)$ such that

$$
\left|E \cap B_{\tau}(x)\right| \geq\left(\frac{\sigma}{2 \xi(n)}\right)^{n+1} \tau^{n+1} .
$$


PROOF. By contradiction one assumes that

$$
\left|E \cap B_{\tau}(x)\right|<\left(\frac{\sigma}{2 \xi(n)}\right)^{n+1} \tau^{n+1} \quad \forall x \in E^{(1)} \backslash I_{\tau}(T) .
$$

Setting $\alpha=\xi(n) / \sigma$ so that $\alpha \geq n+1$, we claim that 2.80 implies the existence, for each $x \in E^{(1)} \backslash I_{\tau}(T)$, of $\tau_{x} \in(0, \tau)$ such that

$$
P\left(E ; B_{\tau_{x}}(x)\right)>\frac{\alpha}{\tau}\left|E \cap B_{\tau_{x}}(x)\right| .
$$

In turn, 2.81 contradicts 2.80): indeed, by applying Besicovitch's theorem to $\left\{\operatorname{cl}\left(B_{\tau_{x}}(x)\right): x \in E^{(1)} \backslash I_{\tau}(T)\right\}$ we find an at most countable subset $I$ of $E^{(1)} \backslash$ $I_{\tau}(T)$ such that $\left\{\operatorname{cl}\left(B_{\tau_{x}}(x)\right)\right\}_{x \in I}$ is disjoint and

$$
\begin{aligned}
\left|E \backslash I_{\tau}(T)\right| & \leq \xi(n) \sum_{x \in I}\left|E \cap B_{\tau_{x}}(x)\right|<\frac{\xi(n) \tau}{\alpha} \sum_{x \in I} P\left(E ; B_{\tau_{x}}(x)\right) \\
& \leq \frac{\xi(n) \tau P(E ; A)}{\alpha}=\tau \sigma P(E ; A) \leq\left|E \backslash I_{\tau}(T)\right|,
\end{aligned}
$$

a contradiction. We show that (2.80) implies $(2.81)$ : indeed, if 2.80$)$ holds but (2.81) fails, then there exists $x \in E^{(1)} \backslash I_{\tau}(T)$ such that, setting $m(r)=\mid E \cap$ $B_{r}(x) \mid$ for $r>0$,

$$
m>0 \text { on }(0, \infty), \quad m(\tau)<\left(\frac{\tau}{2 \alpha}\right)^{n+1},
$$

and $(\alpha / \tau) m(r) \geq P\left(E ; B_{r}(x)\right)$ for every $r \in(0, \tau)$. Adding up $\mathcal{H}^{n}\left(\partial B_{r}(x) \cap E\right)$, which equals $m^{\prime}(r)$ for a.e. $r>0$ by the coarea formula, we obtain

$$
m^{\prime}(r)+\frac{\alpha}{\tau} m(r) \geq P\left(E \cap B_{r}(x)\right) \geq m(r)^{n /(n+1)} \quad \text { for a.e. } r \in(0, \tau) .
$$

where in the last inequality we have used that $P(F) \geq|F|^{n /(n+1)}$ whenever $0<$ $|F|<\infty$; see, e.g., [42, prop. 12.35]. Since $m>0$ on $(0, \infty)$ we find

$$
\begin{gathered}
\left\{\begin{array} { l } 
{ \frac { \alpha } { \tau } m ( r ) \leq ( 1 / 2 ) m ( r ) ^ { n / ( n + 1 ) } } \\
{ \forall r \in ( 0 , \tau ) }
\end{array} \quad \text { iff } \quad \left\{\begin{array}{l}
m(r) \leq(\tau / 2 \alpha)^{n+1} \\
\forall r \in(0, \tau)
\end{array}\right.\right. \\
\text { if } m(\tau) \leq\left(\frac{\tau}{2 \alpha}\right)^{n+1},
\end{gathered}
$$

where the last condition holds by 2.82. Thus 2.83 gives

$$
m^{\prime}(r) \geq(1 / 2) m(r)^{n /(n+1)} \quad \text { for a.e. } r \in(0, \tau) ;
$$

thus $m(\tau) \geq(\tau / 2(n+1))^{n+1} \geq(\tau / 2 \alpha)^{n+1}$ as $\alpha \geq n+1$, a contradiction. 


\subsection{Isoperimetry, lower bounds, and collapsing}

Given an $L^{1}$-converging sequence of sets of finite perimeter $\left\{E_{j}\right\}_{j}$, the boundary of the $L^{1}$-limit set $E$ will be (in general) strictly included in $K=\operatorname{spt} \mu$, where $\mu$ is the weak-star limit of the Radon measures defined by the boundaries of the $E_{j}$ 's. In the next lemma we show that, under some mild bounds on $\mu$ and $E_{j}$, if $\mu$ is absolutely continuous with respect to $\mathcal{H}^{n}\llcorner K$, then the Radon-Nikodým density $\theta$ of $\mu$ is everywhere larger than 1 , and is actually larger than 2 at a.e. point of $K \backslash \partial^{*} E$ (that is, a cancellation can happen only when boundaries are collapsing).

LEMMA 2.11 (Collapsing lemma). Let $K$ be a relatively compact and $\mathcal{H}^{n}$-rectifiable set in $\Omega$, let $E \subset \Omega$ be a set of finite perimeter with $\Omega \cap \partial^{*} E \subset K$, and let $\left\{E_{j}\right\}_{j} \subset \mathcal{E}$ such that $E_{j} \rightarrow E$ in $L_{\mathrm{loc}}^{1}(\Omega)$, and $\mu_{j} \stackrel{*}{\rightarrow} \mu$ as Radon measures in $\Omega$, where $\mu_{j}=\mathcal{H}^{n}\left\llcorner\left(\Omega \cap \partial E_{j}\right)\right.$ and $\mu=\theta \mathcal{H}^{n}\left\llcorner K\right.$ for a Borel function $\theta$. If $\Omega^{\prime} \subset \Omega$ and $r_{*}>0$ are such that for every $x \in K \cap \Omega^{\prime}$ and a.e. $r<r_{*}$ with $B_{r}(x) \Subset \Omega^{\prime}$ we have

$$
\begin{aligned}
\mu\left(B_{r}(x)\right) & \geq c(n) r^{n}, \\
\liminf _{j \rightarrow \infty} \mathcal{H}^{n}\left(B_{r}(x) \cap \partial E_{j}\right) & \leq C(n) \liminf _{j \rightarrow \infty} \mathcal{H}^{n}\left(\partial B_{r}(x) \backslash A_{r, j}^{0}\right),
\end{aligned}
$$

where $A_{r, j}^{0}$ denotes an $\mathcal{H}^{n}$-maximal connected component of $\partial B_{r}(x) \backslash \partial E_{j}$, then $\theta(x) \geq 1$ for $\mathcal{H}^{n}$-a.e. $x \in K \cap \Omega^{\prime}$, and $\theta(x) \geq 2$ for $\mathcal{H}^{n}$-a.e. $x \in\left(K \backslash \partial^{*} E\right) \cap \Omega^{\prime}$.

The bound $\theta \geq 1$ follows by arguing exactly as in [13, proof of theorem 2, step three], and has nothing to do with the fact that the measures $\mu_{j}$ are defined by boundaries; the latter information is in turn crucial in obtaining the bound $\theta \geq 2$ and requires a new argument. For the sake of clarity, we also give the details of the $\theta \geq 1$ bound, which in turn is based on spherical isoperimetry.

LEMMA 2.12 (Spherical isoperimetry). Let $\Sigma \subset \mathbb{R}^{n+1}$ denote a spherical cap ${ }^{5}$ in the $n$-dimensional unit sphere $\mathbb{S}^{n}$, possibly with $\Sigma=\mathbb{S}^{n}$. If $K$ is a compact set in $\mathbb{R}^{n+1}$ and $\left\{A^{h}\right\}_{h=0}^{\infty}$ is the family of the open connected components of $\Sigma \backslash K$, ordered so to have $\mathcal{H}^{n}\left(A^{h}\right) \geq \mathcal{H}^{n}\left(A^{h+1}\right)$, then

$$
\mathcal{H}^{n}\left(\Sigma \backslash A^{0}\right) \leq C(n) \mathcal{H}^{n-1}(\Sigma \cap K)^{n /(n-1)} .
$$

Moreover, if $\Sigma=\mathbb{S}^{n}, \sigma_{n}=\mathcal{H}^{n}\left(\mathbb{S}^{n}\right)$, and $\mathcal{H}^{n-1}\left(\mathbb{S}^{n} \cap K\right)<\infty$, then each $A^{h}$ is a set of finite perimeter in $\mathbb{S}^{n}$ and for every $\tau>0$ there exists $\sigma>0$ such that

$$
\min \left\{\mathcal{H}^{n}\left(A^{0}\right), \mathcal{H}^{n}\left(A^{1}\right)\right\}=\mathcal{H}^{n}\left(A^{1}\right) \geq \frac{\sigma_{n}}{2}-\sigma
$$

implies

$$
\min \left\{\mathcal{H}^{n-1}\left(\partial^{*} A^{0}\right), \mathcal{H}^{n-1}\left(\partial^{*} A^{1}\right)\right\} \geq \sigma_{n-1}-\tau .
$$

Here $\partial^{*} A^{h}$ denotes the reduced boundary of $A^{h}$ in $\mathbb{S}^{n}$.

\footnotetext{
${ }^{5}$ That is, $\Sigma=\mathbb{S}^{n} \cap H$ where $H$ is an open half-space of $\mathbb{R}^{n+1}$.
} 
Proof. This is [13, lemma 9]. However, 2.88) is stated in a weaker form in [13, lemma 9], so we give the details. Arguing by contradiction, we can find $\tau>0$ and $\left\{K_{j}\right\}_{j}$ such that, for $\alpha=0,1, \mathcal{H}^{n-1}\left(\partial^{*} A_{j}^{\alpha}\right) \leq \sigma_{n-1}-\tau$ for every $j$, but $\mathcal{H}^{n}\left(A_{j}^{\alpha}\right) \rightarrow \sigma_{n} / 2$ as $j \rightarrow \infty$. Since $\sigma_{n}=\mathcal{H}^{n}\left(\mathbb{S}^{n}\right)$ and $A_{j}^{0} \cap A_{j}^{1}=\varnothing$, we find that, for $\alpha=0,1, A_{j}^{\alpha} \rightarrow A^{\alpha}$ in $L^{1}\left(\mathbb{S}^{n}\right)$ where $A^{0} \cap A^{1}=\varnothing$ and $A^{0} \cup A^{1}$ is $\mathcal{H}^{n}$ equivalent to $\mathbb{S}^{n}$. Therefore $\mathcal{H}^{n-1}\left(\partial^{*} A^{0}\right)=\mathcal{H}^{n-1}\left(\partial^{*} A^{1}\right) \leq \sigma_{n-1}-\tau$, where we have used lower semicontinuity of the perimeter functional. Since inf $\mathcal{H}^{n-1}\left(\partial^{*} A\right)$ with $\mathcal{H}^{n}(A)=\sigma_{n} / 2$ is equal to $\sigma_{n-1}$, we have reached a contradiction.

PROOF OF LEMMA 2.11.

Step 1. We fix $x \in K \cap \Omega^{\prime}$ such that $\mathcal{H}^{n}\left\llcorner(K-x) / r \stackrel{*}{\rightarrow} \mathcal{H}^{n}\left\llcorner T_{x} K\right.\right.$ as $r \rightarrow 0^{+}$. Setting $v(x)^{\perp}=T_{x} K$ for $v(x) \in \mathbb{S}^{n}$, by the lower density estimate (2.84) we easily find that for every $\sigma>0$ there exists $r_{0}=r_{0}(\sigma, x) \in\left(0, \min \left\{r_{*}, \operatorname{dist}\left(x, \partial \Omega^{\prime}\right)\right\}\right)$ such that $|(y-x) \cdot v(x)|<\sigma r$ for every $y \in K \cap B_{r}(x)$ and every $r<r_{0}$. In particular,

$$
\lim _{j \rightarrow \infty} \mathcal{H}^{n}\left(\partial E_{j} \cap\left\{y \in B_{r}(x):|(y-x) \cdot v(x)|>\sigma r\right\}\right)=0 \quad \text { for every } r \leq r_{0},
$$

and thus by the coarea formula (see [13, eq. (2.13)])

$$
\lim _{j \rightarrow \infty} \mathcal{H}^{n-1}\left(\Sigma_{r, \sigma}^{ \pm} \cap \partial E_{j}\right)=0 \quad \text { for a.e. } r \leq r_{0}
$$

where we have set

$$
\begin{aligned}
& \Sigma_{r, \sigma}^{+}=\left\{y \in \partial B_{r}(x):(y-x) \cdot v(x)>\sigma r\right\}, \\
& \Sigma_{r, \sigma}^{-}=\left\{y \in \partial B_{r}(x):(y-x) \cdot v(x)<-\sigma r\right\} .
\end{aligned}
$$

Let $A_{r, j}^{+}$be an $\mathcal{H}^{n}$-maximal connected component of $\Sigma_{r, \sigma}^{+} \backslash \partial E_{j}$, and define similarly $A_{r, j}^{-}$. Equations 2.89) and 2.86) imply that, for a.e. $r<r_{0}$,

$$
\lim _{j \rightarrow \infty} \mathcal{H}^{n}\left(A_{r, j}^{ \pm}\right)=\mathcal{H}^{n}\left(\Sigma_{r, \sigma}^{ \pm}\right)
$$

Now let $\left\{A_{r, j}^{h}\right\}_{h=0}^{\infty}$ denote the open connected components of $\partial B_{r}(x) \backslash \partial E_{j}$, ordered by decreasing $\mathcal{H}^{n}$-measure. We claim that

$$
\text { if (2.90) holds, then either } A_{r, j}^{+} \text {or } A_{r, j}^{-} \text {is not contained in } A_{r, j}^{0} \text {. }
$$

Indeed, if for some $r$ we have $A_{r, j}^{+} \cup A_{r, j}^{-} \subset A_{r, j}^{0}$, then by 2.85) and 2.90) we find

$$
\begin{aligned}
\mu\left(B_{r}(x)\right) & \leq \liminf _{j \rightarrow \infty} \mu_{j}\left(B_{r}(x)\right) \\
& \leq C(n) \liminf _{j \rightarrow \infty} \mathcal{H}^{n}\left(\partial B_{r} \backslash A_{r, j}^{0}\right) \leq C(n) r^{n} \sigma,
\end{aligned}
$$

a contradiction to (2.84) if $\sigma \leq \sigma_{0}(n)$ for a suitable $\sigma_{0}(n)$. By (2.91) and (2.90),

$$
\min \left\{\mathcal{H}^{n}\left(A_{r, j}^{0}\right), \mathcal{H}^{n}\left(A_{r, j}^{1}\right)\right\} \geq\left(\frac{\sigma_{n}}{2}-C(n) \sigma\right) r^{n} \quad \text { for a.e. } r<r_{0} .
$$


By Lemma 2.12 and 2.93), given $\tau>0$, if $\sigma$ is small enough in terms of $n$ and $\tau$, then

(2.94) $\min \left\{\mathcal{H}^{n-1}\left(\partial^{*} A_{r, j}^{0}\right), \mathcal{H}^{n-1}\left(\partial^{*} A_{r, j}^{1}\right)\right\} \geq\left(\sigma_{n-1}-\tau\right) r^{n-1} \quad$ for a.e. $r<r_{0}$ where $\partial^{*} A_{r, j}^{\alpha}$ is the reduced boundary of $A_{r, j}^{\alpha}$ as a subset of $\partial B_{r}(x)$. Since $A_{r, j}^{0}$ is a connected component of $\partial B_{r}(x) \backslash \partial E_{j}$, we have

$$
\left(\sigma_{n-1}-\tau\right) r^{n-1} \leq \mathcal{H}^{n-1}\left(\partial^{*} A_{r, j}^{0}\right) \leq \mathcal{H}^{n-1}\left(\partial B_{r}(x) \cap \partial E_{j}\right) .
$$

Now if $f_{j}(r)=\mu_{j}\left(B_{r}(x)\right)$ and $f(r)=\mu\left(B_{r}(x)\right)$, then by the coarea formula we easily find that $f_{j} \rightarrow f$ a.e. with $\liminf _{j \rightarrow \infty} f_{j}^{\prime}(r) \leq f^{\prime}(r) \leq D f$, where $D f$ denotes the distributional derivative of $f$. Hence, letting $j \rightarrow \infty$ and $\tau \rightarrow 0^{+}$in (2.95), we obtain $D f \geq \sigma_{n-1} r^{n-1} d r$ on $\left(0, r_{0}\right)$. As $\omega_{n}=n \sigma_{n-1}$, we conclude that $\theta(x) \geq 1$. We stress once more that so far we have just followed the argument of [13, proof of theorem 2, step three].

Step 2. We use the boundary structure to show that $\theta \geq 2 \mathcal{H}^{n}$-a.e. on $\Omega^{\prime} \cap(K \backslash$ $\left.\partial^{*} E\right)$. Since $\left\{E^{(0)}, E^{(1)}, \partial^{*} E\right\}$ is an $\mathcal{H}^{n}$-a.e. partition of $\mathbb{R}^{n+1}$, we can assume that $x \in\left(E^{(0)} \cup E^{(1)}\right) \cap K \cap \Omega^{\prime}$. We consider first the case $x \in E^{(0)}$. Given $\sigma>0$, up to decreasing $r_{0}$,

$$
\sigma r_{0}^{n+1} \geq \lim _{j \rightarrow \infty}\left|E_{j} \cap B_{r_{0}}(x)\right|=\lim _{j \rightarrow \infty} \int_{0}^{r_{0}} \mathcal{H}^{n}\left(E_{j} \cap \partial B_{r}(x)\right) d r .
$$

Let us consider the measurable set $I_{j} \subset\left(0, r_{0}\right)$,

$$
I_{j}=\left\{r \in\left(0, r_{0}\right): A_{r, j}^{0} \cup A_{r, j}^{1} \subset \partial B_{r}(x) \backslash \operatorname{cl}\left(E_{j}\right)\right\} .
$$

We claim that

$$
\mathcal{H}^{n-1}\left(\partial^{*} A_{r, j}^{0} \cap \partial^{*} A_{r, j}^{1}\right)=0 \quad \forall r \in I_{j} .
$$

Indeed, if $r \in I_{j}$, then $A_{r, j}^{0}, A_{r, j}^{1}$, and $\partial B_{r}(x) \cap E_{j}$ are disjoint sets of finite perimeter in $\partial B_{r}(x)$, and in particular

$$
\begin{array}{ll}
v_{A_{r, j}^{0}}=-v_{A_{r, j}^{1}}, & \mathcal{H}^{n-1} \text {-a.e. on } \partial^{*} A_{r, j}^{0} \cap \partial^{*} A_{r, j}^{1}, \\
v_{A_{r, j}^{0}}=-v_{\partial B_{r}(x) \cap E_{j}}, & \mathcal{H}^{n-1} \text {-a.e. on } \partial^{*} A_{r, j}^{0} \cap \partial^{*}\left[\partial B_{r}(x) \cap E_{j}\right], \\
v_{A_{r, j}^{1}}=-v_{\partial B_{r}}(x) \cap E_{j}, & \mathcal{H}^{n-1} \text {-a.e. on } \partial^{*} A_{r, j}^{1} \cap \partial^{*}\left[\partial B_{r}(x) \cap E_{j}\right] .
\end{array}
$$

At the same time, since $\left\{A_{r, j}^{h}\right\}_{h=0}^{\infty}$ are connected components of $\partial B_{r}(x) \backslash \partial E_{j}$,

$$
\partial^{*} A_{r, j}^{h} \subset \partial^{*}\left[\partial B_{r}(x) \cap E_{j}\right] \text { modulo } \mathcal{H}^{n}
$$

and thus $\mathcal{H}^{n-1}$-a.e. on $\partial^{*} A_{r, j}^{0} \cap \partial^{*} A_{r, j}^{1}$, we have

$$
v_{\partial B_{r}(x) \cap E_{j}}=-v_{A_{r, j}^{0}}^{0}=v_{A_{r, j}^{1}}=-v_{\partial B_{r}(x) \cap E_{j}},
$$


a contradiction. By (2.94) and 2.97), given $\tau>0$ and provided $\sigma$ is small enough in terms of $n$ and $\tau$, for a.e. $r \in I_{j}$ we find

$$
\begin{aligned}
f_{j}^{\prime}(r) & \geq \mathcal{H}^{n-1}\left(\partial B_{r}(x) \cap \partial E_{j}\right) \geq \mathcal{H}^{n-1}\left(\partial^{*} A_{r, j}^{0} \cup \partial^{*} A_{r, j}^{1}\right) \\
& =\mathcal{H}^{n-1}\left(\partial^{*} A_{r, j}^{0}\right)+\mathcal{H}^{n-1}\left(\partial^{*} A_{r, j}^{1}\right) \geq 2\left(\sigma_{n-1}-\tau\right) r^{n-1}
\end{aligned}
$$

Hence,

$$
\begin{aligned}
f_{j}\left(r_{0}\right) & \geq 2\left(\sigma_{n-1}-\tau\right) \frac{r_{0}^{n}}{n}-C(n) \int_{\left(0, r_{0}\right) \backslash I_{j}} r^{n-1} d r \\
& \geq 2\left(\sigma_{n-1}-\tau\right) \frac{r_{0}^{n}}{n}-C(n) r_{0}^{1 / n}\left(\int_{\left(0, r_{0}\right) \backslash I_{j}} r^{n} d r\right)^{(n-1) / n} .
\end{aligned}
$$

We notice that for a.e. $r \in\left(0, r_{0}\right) \backslash I_{j}$, 2.93) gives

$$
\mathcal{H}^{n}\left(E_{j} \cap \partial B_{r}(x)\right) \geq \min \left\{\mathcal{H}^{n}\left(A_{r, j}^{0}\right), \mathcal{H}^{n}\left(A_{r, j}^{1}\right)\right\} \geq\left(\frac{\sigma_{n}}{2}-C(n) \sigma\right) r^{n}
$$

so that (2.96) implies

$$
\sigma r_{0}^{n+1} \geq c(n) \limsup _{j \rightarrow \infty} \int_{\left(0, r_{0}\right) \backslash I_{j}} r^{n} d r
$$

If we combine (2.98) and (2.99) and let $j \rightarrow \infty$, then we find

$$
f\left(r_{0}\right)=\lim _{j \rightarrow \infty} f_{j}\left(r_{0}\right) \geq 2\left(\sigma_{n-1}-\tau\right) \frac{r_{0}^{n}}{n}-C(n) r_{0}^{1 / n}\left(\sigma r_{0}^{n+1}\right)^{(n-1) / n} .
$$

Dividing by $r_{0}^{n}$ and letting $r_{0} \rightarrow 0^{+}, \sigma \rightarrow 0^{+}$, and $\tau \rightarrow 0^{+}$, we find $\theta(x) \geq 2$ whenever $x \in E^{(0)} \cap K \cap \Omega^{\prime}$. The case when $x \in E^{(1)}$ is analogous and the details are omitted.

\section{Existence of Generalized Minimizers: Proof of Theorem 1.4}

Given the length of the proof, we provide a short overview. In Step 1, we check that $\psi(\varepsilon)<\infty$ by using the open neighborhoods of a minimizer $S$ of $\ell$ as comparison sets for $\psi(\varepsilon)$. We remark that this is the only point of the proof where (1.12) is used. It is important here to allow for sufficiently nonsmooth sets in the competition class $\mathcal{E}$ : indeed, minimizers of $\ell$ are known to be smooth only outside of a close $\mathcal{H}^{n}$-negligible set in arbitrary dimension. Once $\psi(\varepsilon)<\infty$ is established, we consider a minimizing sequence $\left\{E_{j}\right\}_{j}$ for $\psi(\varepsilon)$, so that $E_{j} \in \mathcal{E},\left|E_{j}\right|=\varepsilon$, $\Omega \cap \partial E_{j}$ is $\mathcal{C}$-spanning $W$, and

$$
\begin{gathered}
\mathcal{H}^{n}\left(\Omega \cap \partial E_{j}\right) \leq \mathcal{H}^{n}(\Omega \cap \partial F)+\frac{1}{j} \\
\forall F \in \mathcal{E}|F|=\varepsilon \Omega \cap \partial F \text { is } \mathcal{C} \text {-spanning } W .
\end{gathered}
$$

We want to apply (3.1) to the comparison sets constructed in Section 2, but, in general, those local variations do not preserve the volume constraint. A family 
of volume-fixing variations acting uniformly on $\left\{E_{j}\right\}_{j}$ is constructed through the nucleation lemma (Lemma 2.10) following some ideas introduced by Almgren in the existence theory of minimizing clusters [1]; see Steps 2 and 3. In Step 4 we exploit cup and cone competitors to show that, up to extracting subsequences, $\mathcal{H}^{n}\left\llcorner\left(\Omega \cap \partial E_{j}\right) \stackrel{*}{\rightarrow} \mu=\theta \mathcal{H}^{n}\left\llcorner K\right.\right.$ as Radon measures in $\Omega$, and $E_{j} \rightarrow E$ in $L_{\text {loc }}^{1}(\Omega)$ for a pair $(K, E) \in \mathcal{K}$ and for an upper semicontinuous function $\theta \geq 1$ on $K$. An application of Lemma 2.11 shows that $\theta \geq 2 \mathcal{H}^{n}$-a.e. on $K \backslash \partial^{*} E$, thus proving $\psi(\varepsilon) \geq \mathcal{F}(K, E)$. In order to show that $\psi(\varepsilon)=\mathcal{F}(K, E)$, and thus that $(K, E)$ is a generalized minimizer of $\psi(\varepsilon)$, we need to exclude that $\Omega \cap \partial E_{j}$ concentrates area by folding against $K$ at infinity or against the wire frame. By using slab competitors we prove that $\Omega \cap \partial E_{j}$, in its convergence towards $K$, cannot fold at all near points in $\partial^{*} E$, and can fold at most twice near points in $K \cap\left(E^{(0)} \cup E^{(1)}\right)$ (Step 5). In Step 6, concentration of area at the boundary is ruled out by a deformation argument based on Lemma 2.4. Finally, in Step 7, we exclude area (and volume) concentration at infinity by using exterior cup competitors to construct a uniformly bounded minimizing sequence.

Proof of Theorem 1.4 .

Step 1 . We show that

$$
\psi(\varepsilon) \leq 2 \ell+C(n) \varepsilon^{n /(n+1)} \quad \forall \varepsilon>0 .
$$

Let $S$ be a minimizer of $\ell$, and let $\eta_{0}>0$ be such that $(1.12)$ holds. If $\eta \in\left(0, \eta_{0}\right)$, then the open $\eta$-neighborhood $U_{\eta}(S)$ of $S$ is such that $\Omega \cap \partial U_{\eta}(S)$ is $\mathcal{C}$-spanning $W$ : otherwise we could find $\eta \in\left(0, \eta_{0}\right)$ and $\gamma \in \mathcal{C}$ such that $\gamma \cap \partial U_{\eta}(S)=\varnothing$. Since $\gamma$ is connected, we would either have $\gamma \subset\{x: \operatorname{dist}(x, S)>\eta\}$, against the fact that $S$ is $\mathcal{C}$-spanning, or we would have $\gamma \subset U_{\eta}(S)$ against 1.12). Hence $\Omega \cap \partial U_{\eta}(S)$ is $\mathcal{C}$-spanning $W$.

As proved in [13], $S$ is $\mathcal{H}^{n}$-rectifiable. Moreover, as shown in Theorem B.1] in the appendix, we have

$$
\mathcal{H}^{n}\left(S \cap B_{r}(x)\right) \geq c(n) r^{n} \quad \forall x \in \operatorname{cl}(S) r<\rho_{0}
$$

where $\rho_{0}$ depends on $W$, so that $\mathcal{H}^{n}(S)<\infty$ implies that $\operatorname{cl}(S)$ is compact. This density estimate has two more consequences: first, combined with [42, cor. 6.5], it implies $\mathcal{H}^{n}(\operatorname{cl}(S) \backslash S)=0$; second, it allows us to exploit [3, theorem 2.104] to find

$$
\left|U_{\eta}(S)\right|=2 \eta \mathcal{H}^{n}(\operatorname{cl}(S))+\mathrm{o}(\eta)=2 \eta \mathcal{H}^{n}(S)+\mathrm{o}(\eta) \quad \text { as } \eta \rightarrow 0^{+} .
$$

By the coarea formula for Lipschitz maps applied to the distance function from $S$ (see [42, theorem 18.1, remark 18.2]), we have

$$
\begin{gathered}
\left|U_{\eta}(S) \cap A\right|=\int_{0}^{\eta} P\left(U_{t}(S) ; A\right) d t=\int_{0}^{\eta} \mathcal{H}^{n}\left(A \cap \partial U_{t}(S)\right) d t \\
\forall A \subset \mathbb{R}^{n+1} \text { open }
\end{gathered}
$$


so that $U_{\eta}(S)$ is a set of finite perimeter in $\mathbb{R}^{n+1}$ and $\mathcal{H}^{n}\left(\partial U_{\eta}(S) \backslash \partial^{*} U_{\eta}(S)\right)=0$ for a.e. $\eta>0$. Summarizing, we have proved that, for a.e. $\eta \in\left(0, \eta_{0}\right)$,

$$
F_{\eta}=\Omega \cap U_{\eta}(S) \in \mathcal{E}, \quad \Omega \cap \operatorname{cl}\left(\partial^{*} F_{\eta}\right)=\Omega \cap \partial F_{\eta} \text { is } \mathcal{C} \text {-spanning } W,
$$

and, by (3.4),

$$
f(\eta)=\left|F_{\eta}\right|=\int_{0}^{\eta} P\left(F_{t} ; \Omega\right) d t=\int_{0}^{\eta} P\left(U_{t}(S) ; \Omega\right) d t \leq 2 \eta \mathcal{H}^{n}(S)+\mathrm{o}(\eta) .
$$

Notice that $f(s)$ is absolutely continuous with $f(\eta)=\int_{0}^{\eta} f^{\prime}(t) d t$ and $f^{\prime}(t)=$ $P\left(F_{t} ; \Omega\right)$ for a.e. $t \in(0, \eta)$. Hence, for every $\eta>0$ there exist $t_{1}(\eta), t_{2}(\eta) \in(0, \eta)$ such that $f^{\prime}\left(t_{1}(\eta)\right) \leq f(\eta) / \eta \leq f^{\prime}\left(t_{2}(\eta)\right)$. Setting $F_{j}=F_{t_{1}\left(\eta_{j}\right)}$ for a suitable $\eta_{j} \rightarrow 0^{+}$, we get

$$
\limsup _{j \rightarrow \infty} P\left(F_{j} ; \Omega\right) \leq 2 \ell
$$

where $\left|F_{j}\right| \rightarrow 0^{+}$. Finally, given $\varepsilon>0$, we pick $j$ such that $\left|F_{j}\right|<\varepsilon$, and construct a competitor for $\psi(\varepsilon)$ by adding to $F_{j}$ a disjoint ball of volume $\varepsilon-\left|F_{j}\right|$. In this way, $\psi(\varepsilon) \leq P\left(F_{j} ; \Omega\right)+C(n)\left(\varepsilon-\left|F_{j}\right|\right)^{n /(n+1)}$, and 3.2 is found by letting $j \rightarrow \infty$.

Since $\psi(\varepsilon)<\infty$, we can now consider a minimizing sequence $\left\{E_{j}\right\}_{j=1}^{\infty}$ for $\psi(\varepsilon)$. Given that $P\left(E_{j}\right) \leq \mathcal{H}^{n}(\partial \Omega)+\mathcal{H}^{n}\left(\Omega \cap \partial E_{j}\right) \leq \mathcal{H}^{n}(\partial \Omega)+\psi(\varepsilon)+1$ for $j$ large, and that $\left|E_{j}\right|=\varepsilon$ for every $j$, there exist a set of finite perimeter $E \subset \Omega$ and a Radon measure $\mu$ in $\Omega$ such that, up to extracting subsequences,

$$
\begin{aligned}
& E_{j} \rightarrow E \text { in } L_{\mathrm{loc}}^{1}(\Omega), \\
& \mu_{j}=\mathcal{H}^{n}\left\llcorner\left(\Omega \cap \partial E_{j}\right) \stackrel{*}{\rightarrow} \mu \quad \text { as Radon measures on } \Omega,\right.
\end{aligned}
$$

as $j \rightarrow \infty$; see, e.g., [42, sec. 12.4]. We consider the set, relatively closed in $\Omega$, defined by

$$
K=\Omega \cap \operatorname{spt} \mu=\left\{x \in \Omega: \mu\left(B_{r}(x)\right)>0 \forall r>0\right\}
$$

and claim that

$$
K \text { is } \mathcal{C} \text {-spanning } W, \quad \Omega \cap \partial^{*} E \subset K .
$$

Indeed, the first claim in (3.6) is obtained by applying Lemma 2.1 to $K_{j}=\Omega \cap$ $\partial E_{j}$; and if $x \in \Omega \cap \partial^{*} E$ and $B_{r}(x) \subset \Omega$, then

$$
0<P\left(E ; B_{r}(x)\right) \leq \liminf _{j \rightarrow \infty} P\left(E_{j} ; B_{r}(x)\right) \leq \liminf _{j \rightarrow \infty} \mu_{j}\left(B_{r}(x)\right) \leq \mu\left(\operatorname{cl}\left(B_{r}(x)\right)\right)
$$

so that $x \in K$. Notice that, at this stage, we still do not know if $(K, E) \in \mathcal{K}$ : we still need to show that $K$ is $\mathcal{H}^{n}$-rectifiable and, possibly up to Lebesgue negligible modifications, that $E$ is open with $\Omega \cap \operatorname{cl}\left(\partial^{*} E\right)=\Omega \cap \partial E$. Moreover, we just have $|E| \leq \varepsilon$ (possible volume loss at infinity), and we know nothing about the structure of $\mu$. 
Step 2. We show the existence of $\tau>0$ such that for every $E_{j}$ there exist $x_{j}^{1}, x_{j}^{2} \in \mathbb{R}^{n+1}$ such that $\left\{\operatorname{cl}\left(B_{2 \tau}\left(x_{j}^{1}\right)\right), \operatorname{cl}\left(B_{2 \tau}\left(x_{j}^{2}\right)\right), W\right\}$ is disjoint and

$$
\left|E_{j} \cap B_{\tau}\left(x_{j}^{1}\right)\right|=\kappa_{1}, \quad\left|E_{j} \cap B_{\tau}\left(x_{j}^{2}\right)\right|=\kappa_{2},
$$

for some $\kappa_{1}, \kappa_{2} \in\left(0,\left|B_{\tau}\right| / 2\right]$ depending on $n, \tau, \varepsilon$, and $\ell$ only. With $\tau_{0}$ as in (1.11), for $M \in \mathbb{N} \backslash\{0\}$ to be chosen later on, and by compactness of $W$, we can pick $\tau>0$ so that

$$
(M+1) \tau<\tau_{0}, \quad\left|B_{M} \tau\right|<\frac{\varepsilon}{4}, \quad\left|I_{(M+1) \tau}(W) \backslash W\right|<\frac{\varepsilon}{2} .
$$

The value $\sigma$ in Lemma 2.10 corresponding to $E_{j}$ and $T=I_{M}(W)$ is given by

$$
\min \left\{\frac{\left|E_{j} \backslash I_{\tau}(T)\right|}{\tau P\left(E_{j} ; \mathbb{R}^{n+1} \backslash T\right)}, \frac{\xi(n)}{n+1}\right\} \geq \min \left\{\frac{\varepsilon / 2}{\tau(\psi(\varepsilon)+1)}, \frac{\xi(n)}{n+1}\right\}>0
$$

since $\left|E_{j} \backslash I_{\tau}(T)\right| \geq \varepsilon / 2$ by (3.8), and since $P\left(E_{j} ; \Omega\right) \leq \psi(\varepsilon)+1$. Therefore, by setting

$$
\sigma_{1}=\min \left\{\frac{\varepsilon / 2}{\tau(\psi(\varepsilon)+1)}, \frac{\xi(n)}{n+1}\right\},
$$

an application of Lemma 2.10 yields $y_{j} \in \mathbb{R}^{n+1} \backslash I_{(M+1) \tau}(W)$ such that

$$
\left|E_{j} \cap B_{\tau}\left(y_{j}\right)\right| \geq \min \left\{\left(\frac{\sigma_{1}}{2 \xi(n)}\right)^{n+1} \tau^{n+1} \frac{\left|B_{\tau}\right|}{2}\right\}=\kappa_{1}
$$

so that $\kappa_{1} \in\left(0,\left|B_{\tau}\right| / 2\right]$ depends on $n, \ell, \varepsilon$, and $\tau$ only (observe that this is a consequence of (3.2)). The continuous map $x \mapsto\left|E_{j} \cap B_{\tau}(x)\right|$ takes a value larger than $\kappa_{1}$ at $y_{j} \in \mathbb{R}^{n+1} \backslash I_{(M+1) \tau}(W)$; at the same time, by (1.11), $\mathbb{R}^{n+1} \backslash I_{(M+1) \tau}(W)$ is open and connected; therefore it is pathwise connected [20, cor. 5.6], and $\mid E_{j} \cap$ $B_{\tau}(x) \mid \rightarrow 0$ as $|x| \rightarrow \infty$ in $\mathbb{R}^{n+1} \backslash I_{(M+1) \tau}(W)$. Thus we can find $x_{j}^{1} \in \mathbb{R}^{n+1} \backslash$ $I_{(M+1) \tau}(W)$ such that the first identity in 3.7$)$ holds and $\left\{\operatorname{cl}\left(B_{(M+1) \tau}\left(x_{j}^{1}\right)\right), W\right\}$ is disjoint. Setting $B=\operatorname{cl}\left(B_{(M-2) \tau}\left(x_{j}^{1}\right)\right)$, the value $\sigma$ in Lemma 2.10 corresponding to $E_{j}$ and $T=I_{\tau}(W) \cup B$ is given by

$$
\min \left\{\frac{\left|E_{j} \backslash I_{\tau}(T)\right|}{\tau P\left(E_{j} ; \mathbb{R}^{n+1} \backslash T\right)}, \frac{\xi(n)}{n+1}\right\} \geq \min \left\{\frac{\varepsilon / 4}{\tau(\psi(\varepsilon)+1)}, \frac{\xi(n)}{n+1}\right\}>0
$$

so that, after setting

$$
\sigma_{2}=\min \left\{\frac{\varepsilon / 4}{\tau(\psi(\varepsilon)+1)}, \frac{\xi(n)}{n+1}\right\},
$$

we can find $z_{j} \in \mathbb{R}^{n+1} \backslash\left(I_{2 \tau}(W) \cup \operatorname{cl}\left(B_{(M-1) \tau}\left(x_{j}^{1}\right)\right)\right)$ such that

$$
\left|E_{j} \cap B_{\tau}\left(z_{j}\right)\right| \geq \min \left\{\left(\frac{\sigma_{2}}{2 \xi(n)}\right)^{n+1} \tau^{n+1} \frac{\left|B_{\tau}\right|}{2}\right\}=\kappa_{2}
$$

with $\kappa_{2} \in\left(0,\left|B_{\tau}\right| / 2\right]$ depending on $n, \ell, \varepsilon$, and $\tau$ only. Since $I_{2 \tau}(W)$ and $\operatorname{cl}\left(B_{(M-1) \tau}\left(x_{j}^{1}\right)\right)$ are disjoint and since $\mathbb{R}^{n+1} \backslash I_{2 \tau}(W)$ is pathwise connected by 
(1.11), we easily check that $\mathbb{R}^{n+1} \backslash\left(I_{2 \tau}(W) \cup \operatorname{cl}\left(B_{(M-1) \tau}\left(x_{j}^{1}\right)\right)\right)$ is pathwise connected. By continuity,

$$
\exists x_{j}^{2} \in \mathbb{R}^{n+1} \backslash\left(I_{2 \tau}(W) \cup \operatorname{cl}\left(B_{(M-1) \tau}\left(x_{j}^{1}\right)\right)\right)
$$

such that the second identity in (3.7) holds. Finally, (3.9) implies that the family of sets

$$
\left\{\operatorname{cl}\left(B_{(M-3) \tau}\left(x_{j}^{1}\right)\right), \operatorname{cl}\left(B_{2 \tau}\left(x_{j}^{2}\right)\right), W\right\}
$$

is disjoint. We pick $M=5$ to conclude the proof.

Step 3. In this step we show that 3.1) can be modified to allow for comparison with local variations $F_{j}$ of $E_{j}$ that do not necessarily preserve the volume constraint. More precisely, we prove the existence of positive constants $r_{*}$ and $C_{*}$ (depending on the whole sequence $\left\{E_{j}\right\}_{j}$, and thus uniform in $j$ ) such that if $x \in \Omega, r<r_{*}$, and $\left\{F_{j}\right\}_{j}$ is an admissible local variation of $\left\{E_{j}\right\}_{j}$ in $B_{r}(x)$, in the sense that

$$
F_{j} \in \mathcal{E}, \quad F_{j} \Delta E_{j} \Subset B_{r}(x), \quad \Omega \cap \partial F_{j} \text { is } \mathcal{C} \text {-spanning } W
$$

(notice that we do not require $B_{r}(x) \subset \Omega$ ), then

$$
\mathcal{H}^{n}\left(\Omega \cap \partial E_{j}\right) \leq \mathcal{H}^{n}\left(\Omega \cap \partial F_{j}\right)+C_{*}|| E_{j}|-| F_{j}||+\frac{1}{j} .
$$

We first claim that if $B_{j} \subset \Omega$ is a ball with $\operatorname{dist}\left(B_{j}, B_{r}(x)\right)>0, \zeta: \Omega \rightarrow \Omega$ is a diffeomorphism with $\zeta\left(B_{j}\right) \subset B_{j}$, and $\{\zeta \neq \mathrm{id}\} \Subset B_{j}$, and if

$$
G_{j}=\left(F_{j} \cap B_{r}(x)\right) \cup\left(\zeta\left(E_{j}\right) \cap B_{j}\right) \cup\left(E_{j} \backslash\left(B_{j} \cup B_{r}(x)\right)\right)
$$

then $G_{j} \in \mathcal{E}$ and $\Omega \cap \partial G_{j}$ is $\mathcal{C}$-spanning $W$. The fact that $G_{j}$ is open is obvious since $G_{j}$ is equal to $E_{j}$ in a neighborhood of $\Omega \backslash\left(B_{r}(x) \cup B_{j}\right)$, to $F_{j}$ in a neighborhood of $B_{r}(x)$, and to $\zeta\left(E_{j}\right)$ in a neighborhood of $B_{j}$, where $E_{j}, F_{j}$, and $\zeta\left(E_{j}\right)$ are open, and where $\operatorname{dist}\left(B_{j}, B_{r}(x)\right)>0$; this also shows that $\partial G_{j}$ is equal to $\partial E_{j}$ in a neighborhood of $\Omega \backslash\left(B_{r}(x) \cup B_{j}\right)$, to $\partial F_{j}$ in a neighborhood of $B_{r}(x)$, and to $\partial \zeta\left(E_{j}\right)=\zeta\left(\partial E_{j}\right)$ in a neighborhood of $B_{j}$, so that $\Omega \cap \partial G_{j}$ is $\mathcal{H}^{n}$-rectifiable and, thanks to 3.10) and Lemma 2.3, that $\Omega \cap \partial G_{j}$ is $\mathcal{C}$-spanning $W$. Having proved the claim, we only have to construct sets $G_{j}$ as in (3.12) and such that

$$
\left|G_{j}\right|=\varepsilon, \quad \mathcal{H}^{n}\left(\Omega \cap \partial G_{j}\right) \leq \mathcal{H}^{n}\left(\Omega \cap \partial F_{j}\right)+C_{*}|| E_{j}|-| F_{j}||
$$

in order to deduce 3.11) from 3.1. To this aim, let $\left\{x_{j}^{k}\right\}_{k=1,2}$ be as in Step 2: the sets $\left\{\left(E_{j}-x_{j}^{k}\right) \cap B_{\tau}(0)\right\}_{j}$ are bounded in $B_{\tau}(0)$ and have uniformly bounded perimeters, so that, up to extracting a subsequence, for each $k=1,2$ there exists a set of finite perimeter $E_{*}^{k} \subset B_{\tau}(0)$ such that $\left(E_{j}-x_{j}^{k}\right) \cap B_{\tau}(0) \rightarrow E_{*}^{k}$ in $L^{1}\left(\mathbb{R}^{n+1}\right)$. The crucial point is that, by (3.7) and since $\kappa_{k} \in\left(0,\left|B_{\tau}(0)\right| / 2\right]$, we must have

$$
B_{\tau}(0) \cap \partial^{*} E_{*}^{k} \neq \varnothing .
$$


Hence, by arguing as in [42, sec. 29.6], we can find positive constants $C_{*}^{\prime}$ and $\varepsilon_{*}$ such that for every set of finite perimeter $E^{\prime} \subset B_{\tau}(0)$ with

$$
\left|E^{\prime} \Delta E_{*}^{k}\right|<\varepsilon_{*}
$$

there exists a $C^{1}$-map $\Phi_{k}:\left(-\varepsilon_{*}, \varepsilon_{*}\right) \times B_{\tau}(0) \rightarrow B_{\tau}(0)$ such that, for each $|v|<\varepsilon_{*}$ : (i) $\Phi_{k}(v, \cdot)$ is a diffeomorphism with $\left\{\Phi_{k}(v, \cdot) \neq \mathrm{Id}\right\} \Subset B_{\tau}(0)$; (ii) $\left|\Phi_{k}\left(v, E^{\prime}\right)\right|=\left|E^{\prime}\right|+v$; (iii) if $\Sigma$ is an $\mathcal{H}^{n}$-rectifiable set in $B_{\tau}(0)$, then

$$
\left|\mathcal{H}^{n}\left(\Phi_{k}(v, \Sigma)\right)-\mathcal{H}^{n}(\Sigma)\right| \leq C_{*}^{\prime} \mathcal{H}^{n}(\Sigma)|v| \text {. }
$$

By taking $E^{\prime}=\left(E_{j}-x_{j}^{k}\right) \cap B_{\tau}(0)$ (for $j$ large enough), by composing the maps $\Phi_{k}$ with a translation by $x_{j}^{k}$, and then by extending the resulting maps as the identity map outside of $B_{\tau}\left(x_{j}^{k}\right)$, we prove the existence of $C^{1}$-maps $\Psi_{k}:\left(-\varepsilon_{*}, \varepsilon_{*}\right) \times$ $\mathbb{R}^{n+1} \rightarrow \mathbb{R}^{n+1}$ such that, for each $|v|<\varepsilon_{*}$ : (i) $\Psi_{k}(v, \cdot)$ is a diffeomorphism with $\left\{\Psi_{k}(v, \cdot) \neq \mathrm{Id}\right\} \Subset B_{\tau}\left(x_{j}^{k}\right)$; (ii) $\left|\Psi_{k}\left(v, E_{j}\right)\right|=\left|E_{j}\right|+v$; (iii) if $\Sigma$ is an $\mathcal{H}^{n}$-rectifiable set in $\mathbb{R}^{n+1}$, then

$$
\left|\mathcal{H}^{n}\left(\Psi_{k}(v, \Sigma)\right)-\mathcal{H}^{n}(\Sigma)\right| \leq C_{*}^{\prime} \mathcal{H}^{n}(\Sigma)|v| .
$$

Finally, we set

$$
r_{*}=\min \left\{\tau,\left(\frac{\varepsilon_{*}}{2 \omega_{n+1}}\right)^{1 /(n+1)}\right\}, \quad B_{j}=B_{\tau}\left(x_{j}^{k(j)}\right),
$$

where $k=k(j) \in\{1,2\}$ is selected so that $\operatorname{dist}\left(B_{r}(x), B_{j}\right)>0$ (this is possible because $r_{x} * \leq \tau$ and $\left\{\operatorname{cl}\left(B_{2 \tau}\left(x_{j}^{1}\right)\right), \operatorname{cl}\left(B_{2 \tau}\left(x_{j}^{2}\right)\right)\right\}$ are disjoint). We finally define $G_{j}$ by 3.12 with

$$
\zeta=\Psi_{k(j)}\left(v_{j}, \cdot\right), \quad v_{j}=\left|E_{j} \cap B_{r}(x)\right|-\left|F_{j} \cap B_{r}(x)\right|,
$$

as we are allowed to do since $E_{j} \Delta F_{j} \Subset B_{r}(x)$ and thus $\left|v_{j}\right| \leq \omega_{n+1} r_{*}^{n+1} \leq$ $\varepsilon_{*} / 2$. To prove (3.13): first, we have $G_{j} \Delta F_{j} \Subset \Omega \backslash \operatorname{cl}\left(B_{r}(x)\right)$, while property (ii) of $\Psi_{k(j)}$ gives

$$
\begin{aligned}
\left|G_{j}\right|-\left|E_{j}\right| & =\left|\Psi_{k(j)}\left(v_{j}, E_{j}\right) \cap B_{j}\right|+\left|F_{j} \cap B_{r}(x)\right|-\left|E_{j} \cap B_{j}\right|-\left|E_{j} \cap B_{r}(x)\right| \\
& =\left|\Psi_{k(j)}\left(v_{j}, E_{j}\right) \cap B_{j}\right|-v_{j}-\left|E_{j} \cap B_{j}\right|=0 ;
\end{aligned}
$$

second, property (iii) applied to the $\mathcal{H}^{n}$-rectifiable set $\Sigma=B_{j} \cap \partial E_{j}$ gives

$$
\begin{aligned}
& \mathcal{H}^{n}\left(\Omega \cap \partial G_{j}\right)-\mathcal{H}^{n}\left(\Omega \cap \partial F_{j}\right) \\
& \quad=\mathcal{H}^{n}\left(\Psi_{k(j)}\left(v_{j}, B_{j} \cap \partial E_{j}\right)\right)-\mathcal{H}^{n}\left(B_{j} \cap \partial E_{j}\right) \leq C_{*}^{\prime}\left|v_{j}\right| \mathcal{H}^{n}\left(B_{j} \cap \partial E_{j}\right)
\end{aligned}
$$

so that 3.13 follows by taking $C_{*}=C_{*}^{\prime}(\psi(\varepsilon)+1)$.

Step 4. In this step we apply (3.11) to the cup and cone competitors constructed in Section 2 and show that $K=\Omega \cap \operatorname{spt} \mu$ is relatively compact in $\Omega$ and $\mathcal{H}^{n}$ rectifiable, that $\mu=\theta \mathcal{H}^{n}\left\llcorner K\right.$ with $\theta \geq 1$ on $K$ and $\theta \geq 2 \mathcal{H}^{n}$-a.e. on $K \backslash \partial^{*} E$, 
and, finally, that $(K, E) \in \mathcal{K}$. To this end, pick $x \in K$, set $d(x)=\operatorname{dist}(x, W)>0$, and let

$$
\begin{aligned}
f_{j}(r) & =\mu_{j}\left(B_{r}(x)\right)=\mathcal{H}^{n}\left(B_{r}(x) \cap \partial E_{j}\right), \quad \text { for every } r \in(0, d(x)) . \\
f(r) & =\mu\left(B_{r}(x)\right),
\end{aligned}
$$

Denoting by $D f$ the distributional derivative of $f$, and by $f^{\prime}$ its classical derivative, the coarea formula (see [13, step one, proof of theorem 2] and [25, theorem 2.9.19]) gives

$$
\begin{gathered}
f_{j} \rightarrow f \text { a.e. on }(0, d(x)), \quad D f_{j} \geq f_{j}^{\prime} d r \\
D f \geq f^{\prime} d r, \quad f^{\prime} \geq g=\liminf _{j \rightarrow \infty} f_{j}^{\prime}, \\
f_{j}^{\prime}(r) \geq \mathcal{H}^{n-1}\left(\partial B_{r}(x) \cap \partial E_{j}\right) \quad \forall j \text { and for a.e. } r \in(0, d(x)) .
\end{gathered}
$$

Now let $\eta \in(0, r / 2)$, let $A_{j}$ denote an $\mathcal{H}^{n}$-maximal open connected component of $\partial B_{r}(x) \backslash \partial E_{j}$, and let $F_{j}$ be the cup competitor defined by $E_{j}$ and $A_{j}$ as in Lemma 2.5. More precisely, when $E_{j} \cap A_{j}=\varnothing$, we let $\left\{\eta_{k}^{j}\right\}_{k=1}^{\infty}$ be the decreasing sequence with $\lim _{k \rightarrow \infty} \eta_{k}^{j}=0$ defined in step two of the proof of Lemma 2.5. and setting, for $\eta_{k}^{j}$ such that $\eta \in\left(\eta_{k+1}^{j}, \eta_{k}^{j}\right]$,

$$
\begin{aligned}
Y_{j} & =\partial B_{r}(x) \backslash\left(\operatorname{cl}\left(E_{j} \cap \partial B_{r}(x)\right) \cup \operatorname{cl}\left(A_{j}\right)\right), \\
S_{j} & =\partial E_{j} \cap \operatorname{cl}\left(A_{j}\right) \backslash\left(\operatorname{cl}\left(E_{j} \cap \partial B_{r}(x)\right) \cup \operatorname{cl}\left(Y_{j}\right)\right), \\
U_{j} & =\partial B_{r}(x) \cap\left\{\mathrm{d}_{S_{j}}<\eta_{k}^{j}\right\},
\end{aligned}
$$

we define

$$
F_{j}=\left(E_{j} \backslash \operatorname{cl}\left(B_{r}(x)\right)\right) \cup N_{\eta_{k}^{j}}\left(Z_{j}\right), \quad Z_{j}=Y_{j} \cup\left(U_{j} \backslash \operatorname{cl}\left(E_{j} \cap \partial B_{r}(x)\right)\right) .
$$

When $A_{j} \subset E_{j}$, instead we define

$$
F_{j}=\left(E_{j} \cup B_{r}(x)\right) \backslash \operatorname{cl}\left(N_{\eta}\left(Y_{j}\right)\right), \quad Y_{j}=\left(E_{j} \cap \partial B_{r}(x)\right) \backslash \operatorname{cl}\left(A_{j}\right) ;
$$

see Figure 2.1. In both cases, $\left\{F_{j}\right\}_{j}$ is an admissible local variation of $\left\{E_{j}\right\}_{j}$ in $B_{r^{\prime}}(x)$ for some $r^{\prime}>r$, and by 2.4), for a.e. $r<d(x)$ we have

$$
\limsup _{\eta \rightarrow 0^{+}} \mathcal{H}^{n}\left(\Omega \cap \partial F_{j}\right) \leq \mathcal{H}^{n}\left(\partial E_{j} \backslash B_{r}(x)\right)+2 \mathcal{H}^{n}\left(\partial B_{r}(x) \backslash A_{j}\right)
$$

so that, by 3.11 , for a.e. $r<\min \left\{d(x), r_{*}\right\}$, we have

$$
f_{j}(r) \leq 2 \mathcal{H}^{n}\left(\partial B_{r}(x) \backslash A_{j}\right)+C_{*} \limsup _{\eta \rightarrow 0^{+}}|| E_{j}|-| F_{j}||+\frac{1}{j} .
$$

The estimate of ||$E_{j}|-| F_{j}||$ is different depending on whether $F_{j}$ is given by (3.16) or by (3.17). In both cases we make use of the Euclidean isoperimetric inequality

$$
(n+1)\left|B_{1}\right|^{1 /(n+1)}|U|^{n /(n+1)} \leq P(U) \quad \forall U \subset \mathbb{R}^{n+1},
$$


and we also need the perimeter identities

$$
\begin{aligned}
& P\left(E_{j} \cap B_{r}(x)\right)=P\left(E_{j} ; B_{r}(x)\right)+\mathcal{H}^{n}\left(E_{j} \cap \partial B_{r}(x)\right), \\
& P\left(B_{r}(x) \backslash E_{j}\right)=P\left(E_{j} ; B_{r}(x)\right)+\mathcal{H}^{n}\left(\partial B_{r}(x) \backslash E_{j}\right),
\end{aligned}
$$

which hold for a.e. $r>0$, with the exceptional set of $r$-values that can be made independent from $j$. We now take $F_{j}$ as in (3.16): up to further decreasing the value of $r_{*}$ so to entail $C_{*} r_{*} /(n+1) \leq 1 / 2$, and assuming that $r<r_{*}$, we have

$$
\begin{aligned}
C_{*}|| E_{j}|-| F_{j}|| & \leq C_{*}\left|E_{j} \cap B_{r}(x)\right|+C_{*} c(n) r^{n} \eta_{k}^{j} \\
& \leq C_{*}\left|B_{1}\right|^{1 /(n+1)} r\left|E_{j} \cap B_{r}(x)\right|^{n /(n+1)}+C_{*} c(n) r^{n} \eta_{k}^{j} \\
& \leq \frac{C_{*}}{n+1} r_{*} P\left(E_{j} \cap B_{r}(x)\right)+C_{*} c(n) r^{n} \eta_{k}^{j} \\
& \leq \frac{1}{2}\left\{P\left(E_{j} ; B_{r}(x)\right)+\mathcal{H}^{n}\left(E_{j} \cap \partial B_{r}(x)\right)\right\}+C_{*} c(n) r^{n} \eta_{k}^{j} \\
& \leq \frac{1}{2}\left\{f_{j}(r)+\mathcal{H}^{n}\left(\partial B_{r}(x) \backslash A_{j}\right)\right\}+C_{*} c(n) r^{n} \eta_{k}^{j},
\end{aligned}
$$

where in the last inequality we have used $\partial^{*} E_{j} \subset \partial E$ and $A_{j} \cap E_{j}=\varnothing$ (that is the assumption under which $F_{j}$ is chosen as in (3.16). If instead we take $F_{j}$ as in (3.17), then

$$
\begin{aligned}
C_{*} & || E_{j}|-| F_{j}|| \\
& =C_{*}|| E_{j} \cap B_{r}(x)|-| F_{j} \cap B_{r}(x)|b i g| \\
& =C_{*}|| B_{r}(x) \backslash E_{j}|-| B_{r}(x) \backslash F_{j}|| \\
& \leq C_{*}\left|B_{1}\right|^{1 /(n+1)} r\left|B_{r}(x) \backslash E_{j}\right|^{n /(n+1)}+C_{*}\left|N_{\eta}\left(\partial B_{r}(x) \cap E_{j} \backslash \operatorname{cl}\left(A_{j}\right)\right)\right| \\
& \leq \frac{1}{2}\left\{P\left(E_{j} ; B_{r}(x)\right)+\mathcal{H}^{n}\left(\partial B_{r}(x) \backslash E_{j}\right)\right\}+C_{*} c(n) r^{n} \eta \\
& \leq \frac{1}{2}\left\{f_{j}(r)+\mathcal{H}^{n}\left(\partial B_{r}(x) \backslash A_{j}\right)\right\}+C_{*} c(n) r^{n} \eta,
\end{aligned}
$$

where in the last inequality we have used $\partial^{*} E_{j} \subset \partial E_{j}$ and $A_{j} \subset E_{j}$ (the assumption corresponding to (3.17). By combining (3.18) with (3.20) and (3.21), we conclude that

$$
\frac{f_{j}(r)}{2} \leq 3 \mathcal{H}^{n}\left(\partial B_{r}(x) \backslash A_{j}\right)+\frac{1}{j} \quad \text { for a.e. } r<\min \left\{r_{*}, d(x)\right\} .
$$

By the spherical isoperimetric inequality, Lemma 2.12, and by (3.15), for a.e. $r<$ $d(x)$,

$$
\mathcal{H}^{n}\left(\partial B_{r}(x) \backslash A_{j}\right) \leq C(n) \mathcal{H}^{n-1}\left(\partial B_{r}(x) \cap \partial E_{j}\right)^{n /(n-1)} \leq C(n) f_{j}^{\prime}(r)^{n /(n-1)},
$$

which combined with (3.22) and (3.14), allows us to conclude (letting $j \rightarrow \infty$ ) that

$$
f(r) \leq C(n) f^{\prime}(r)^{n /(n-1)} \quad \text { for a.e. } r<\min \left\{r_{*}, d(x)\right\}
$$


Since $x \in \operatorname{spt} \mu, f$ is positive, and thus (3.23) implies the existence of $\theta_{0}(n)>0$ such that

$$
\mu\left(B_{r}(x)\right) \geq \theta_{0} \omega_{n} r^{n} \quad \forall x \in K, r<r_{*}, B_{r}(x) \Subset \Omega .
$$

Since $K=\Omega \cap \operatorname{spt} \mu$, by [45, theorem 6.9] and (3.24), we obtain

$$
\mu \geq \theta_{0} \mathcal{H}^{n}\llcorner K \quad \text { on } \Omega \text {. }
$$

As a consequence of $\mu(\Omega)<\infty$ and of (3.24) we deduce that $K$ is bounded, thus relatively compact in $\Omega$. In turn, $\partial^{*} E \subset K$ implies the boundedness of $E$. Notice that we have not excluded $|E|<\varepsilon$ yet.

To further progress in the analysis of $\mu$, given $\eta \in(0, r / 2)$ let use now denote by $F_{j}$ the set corresponding to $\eta$ constructed in Lemma 2.9, so that, by (2.71), for a.e. $r<d(x)$,

$$
\limsup _{\eta \rightarrow 0^{+}} \mathcal{H}^{n}\left(\Omega \cap \partial F_{j}\right) \leq \mathcal{H}^{n}\left(\partial E_{j} \backslash B_{r}(x)\right)+\frac{r}{n} \mathcal{H}^{n-1}\left(\partial E_{j} \cap \partial B_{r}(x)\right) .
$$

Using that $\left\{F_{j}\right\}_{j}$ is an admissible local variation of $\left\{E_{j}\right\}_{j}$ in $B_{r}(x)$, and combining (3.11) and (3.26) with ||$E_{j}|-| F_{j}|| \leq C(n) r^{n+1}$, we find that

$$
\mathcal{H}^{n}\left(B_{r}(x) \cap \partial E_{j}\right) \leq \frac{r}{n} f_{j}^{\prime}(r)+C_{*} r^{n+1}+\frac{1}{j},
$$

so that, as $j \rightarrow \infty, f(r) \leq(r / n) f^{\prime}(r)+C_{*} r^{n+1}$. By combining this last inequality with $D f \geq f^{\prime}(r) d r$ and (3.24), we find that

$$
\begin{aligned}
D & \left(e^{\Lambda r} f(r) / r^{n}\right) \\
& =\frac{n e^{\Lambda r}}{r^{n+1}}\left\{\frac{r}{n} D f+\left(\frac{r \Lambda}{n} f(r)-f(r)\right) d r\right\} \\
& \geq \frac{n e^{\Lambda r}}{r^{n+1}}\left\{f(r)-C_{*} r^{n+1}+\frac{r \Lambda}{n} f(r)-f(r)\right\} d r \\
& =\frac{n e^{\Lambda r}}{r^{n}}\left\{-C_{*} r^{n}+\frac{\Lambda f(r)}{n}\right\} d r \geq n e^{\Lambda r}\left\{-C_{*}+\frac{\Lambda \theta_{0} \omega_{n}}{n}\right\} d r
\end{aligned}
$$

so that, setting $\Lambda \geq n C_{*} /\left(\theta_{0} \omega_{n}\right)$, we have proved

$$
e^{\Lambda r} \frac{\mu\left(B_{r}(x)\right)}{r^{n}} \text { is nondecreasing on } r<\min \left\{r_{*}, d(x)\right\} .
$$

By (3.27) and 3.25) we find that

$$
\theta(x)=\lim _{r \rightarrow 0^{+}} \frac{\mu\left(B_{r}(x)\right)}{\omega_{n} r^{n}} \quad \text { exists in }(0, \infty) \text { for every } x \in K .
$$

By Preiss' theorem, $\mu=\theta \mathcal{H}^{n}\left\llcorner K^{*}\right.$ for a Borel function $\theta$ and a countably $\mathcal{H}^{n}$ rectifiable set $K^{*} \subset \Omega$. Since $K=\Omega \cap$ spt $\mu$, we have $\mathcal{H}^{n}\left(K^{*} \backslash K\right)=0$, while (3.25) gives $\mathcal{H}^{n}\left(K \backslash K^{*}\right)=0$. Thus $K$ is countably $\mathcal{H}^{n}$-rectifiable and $\mu=\theta \mathcal{H}^{n}\llcorner K$. Moreover, $\theta$ is upper semicontinuous on $K$ thanks to 3.27). 
Finally, consider the open set

$$
E^{*}=\left\{x \in \Omega: \exists r>0 \text { s.t. }\left|B_{r}(x)\right|=\left|E \cap B_{r}(x)\right|\right\} .
$$

The topological boundary of $E^{*}$ is equal to

$$
\partial E^{*}=\left\{x \in \operatorname{cl}(\Omega): 0<\left|E \cap B_{r}(x)\right|<\left|B_{r}(x)\right| \forall r>0\right\}
$$

so that $\Omega \cap \operatorname{cl}\left(\partial^{*} E\right)=\Omega \cap \partial E^{*}$ by [42, prop. 12.19]. Clearly $E^{*} \subset E^{(1)}$; moreover, if $x \in E^{(1)} \backslash E^{*}$, then $0<\left|E \cap B_{r}(x)\right|<\left|B_{r}(x)\right|$ for every $r>0$, and thus $x \in \partial E^{*}$. In particular,

$$
\Omega \cap\left(E^{(1)} \backslash E^{*}\right) \subset \Omega \cap \partial E^{*}=\Omega \cap \operatorname{cl}\left(\partial^{*} E\right) \subset K,
$$

where $K$ is $\mathcal{H}^{n}$-rectifiable, and thus Lebesgue negligible. Since $\mathcal{H}^{n}(\partial \Omega)<\infty$, we have proved $\mathcal{H}^{n}\left(E^{(1)} \backslash E^{*}\right)<\infty$, and thus $\left|E^{(1)} \Delta E^{*}\right|=0$. By Lebesgue's points theorem, $E^{*}$ is equivalent to $E$, so that $\partial^{*} E=\partial^{*} E^{*}$. Replacing $E$ with $E^{*}$ we find $(K, E) \in \mathcal{K}$. Finally, the lower bounds $\theta \geq 1 \mathcal{H}^{n}$-a.e. on $K$ and $\theta \geq 2$ $\mathcal{H}^{n}$-a.e. on $K \backslash \partial^{*} E$ follow by applying Lemma 2.11 with $\Omega^{\prime}=\Omega$ : notice indeed that assumptions (2.84) and (2.85) in Lemma 2.11 hold by (3.24) and by (3.22).

Step 5. We show that $\theta(x) \leq 1$ at every $x \in \Omega \cap \partial^{*} E$ and that $\theta(x) \leq 2$ at every $x \in K \cap\left(E^{(0)} \cup E^{(1)}\right)$ such that $K$ admits an approximate tangent plane at $x$ (thus, that $\theta \leq 2 \mathcal{H}^{n}$-a.e. on $K \backslash \partial^{*} E$ ). We choose $v(x) \in \mathbb{S}^{n}$ such that $T_{x} K=v(x)^{\perp}$ (notice that, necessarily, $v(x)=v_{E}(x)$ or $v(x)=-v_{E}(x)$ when, in addition, $\left.x \in \partial^{*} E\right)$, and let $B_{2 r}(x) \Subset \Omega$. For $\tau \in(0,1)$ and $\sigma \in(0, \tau)$ we set

$$
\begin{aligned}
S_{\tau, r} & =\left\{y \in B_{r}(x):|(y-x) \cdot v(x)|<\tau r\right\}, \\
V_{\sigma, r} & =\left\{y \in B_{r}(x):|(y-x) \cdot v(x)|<\sigma|y-x|\right\} \subset S_{\sigma, r} \subset S_{\tau, r}, \\
W_{\tau, \sigma, r}^{ \pm} & =\left(S_{\tau, r} \backslash \operatorname{cl}\left(V_{\sigma, r}\right)\right) \cap\left\{y:(y-x) \cdot v_{E}(x) \gtrless 0\right\}, \\
\Gamma_{\tau, \sigma, r}^{ \pm} & =\partial S_{\tau, r} \cap \partial W_{\tau, \sigma, r}^{ \pm},
\end{aligned}
$$

that are depicted in Figure 3.1. By (3.24) and since $\mathcal{H}^{n}\left\llcorner(K-x) / \rho \stackrel{*}{\rightarrow} \mathcal{H}^{n}\left\llcorner T_{x} K\right.\right.$ as $\rho \rightarrow 0^{+}$, the approximate tangent plane $T_{x} K$ is a classical tangent plane, and thus there exists $r_{0}=r_{0}(\sigma, x)>0$ such that $K \cap B_{r}(x) \subset S_{\sigma, r}$ for every $r<r_{0}$, or, equivalently,

$$
K \cap B_{r_{0}}(x) \subset V_{\sigma, r_{0}} \cup\{x\}
$$

In particular,

$$
\mu\left(S_{\tau, r}\right)=\mu\left(B_{r}(x)\right) \quad \forall r<r_{0} .
$$

We also notice that for a.e. value of $r$ we have

$$
\partial S_{\tau, r} \cap \partial E_{j} \text { is } \mathcal{H}^{n-1} \text {-rectifiable } \forall j .
$$




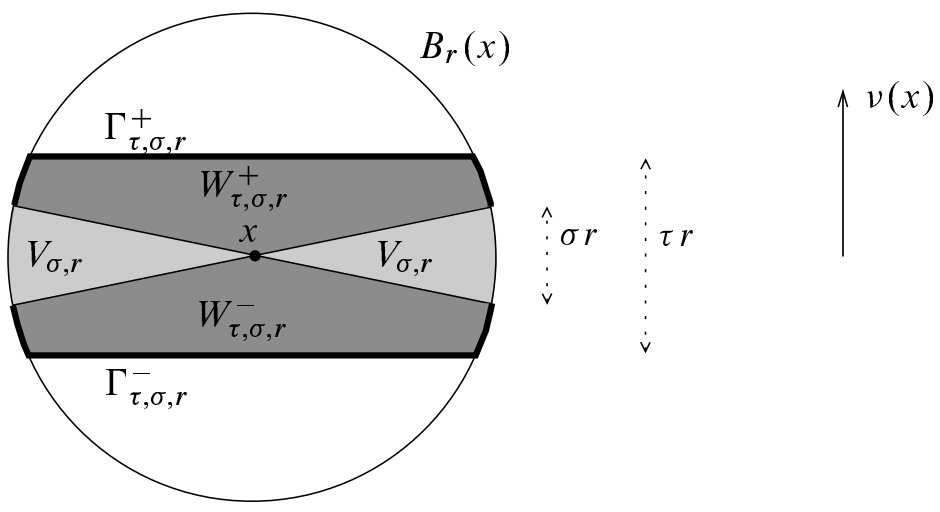

FIGURE 3.1. The sets defined in (3.28). Here $\sigma<\tau<1$, and $S_{\tau, r}$ is decomposed into a central open cone $V_{\sigma, r}$ of small amplitude $\sigma$, the upper and lower open regions $W_{\tau, \sigma, r}^{ \pm}$, and the closed cone $S_{\tau, r} \cap \partial V_{\sigma, r}$. For $r \leq r_{0}(\sigma, x), B_{r}(x) \cap K$ lies inside $V_{\sigma, r}$ by approximate differentiability of $K$ at $x$ and by the density estimate (3.24). When $x \in \partial^{*} E$, if we choose $v(x)=v_{E}(x)$, then the divergence theorem implies that $E$ fills up the whole $W_{\tau, \sigma, r}^{-}$, and leaves empty $W_{\tau, \sigma, r}^{+}$.

We now introduce the family of open sets

$$
\begin{aligned}
& \mathcal{A}_{r, j}^{\text {out }}=\left\{A \subset \partial S_{\tau, r}: A\right. \text { is an open connected component } \\
& \text { of } \left.\partial S_{\tau, r} \backslash \partial E_{j} \text { and } A \text { is disjoint from } E_{j}\right\} \text {, } \\
& \mathcal{A}_{r, j}^{\text {in }}=\left\{A \subset \partial S_{\tau, r}: A\right. \text { is an open connected component } \\
& \text { of } \left.\partial S_{\tau, r} \backslash \partial E_{j} \text { and } A \text { is contained in } E_{j}\right\} \text {, }
\end{aligned}
$$

and denote by $A_{r, j}^{\text {out }}$ and $A_{r, j}^{\text {in }} \mathcal{H}^{n}$-maximal elements of $\mathcal{A}_{r, j}^{\text {out }}$ and $\mathcal{A}_{r, j}^{\text {in }}$ respectively. Finally, given $\eta \in(0, r / 2)$, we let $F_{j}^{\star}$ be the slab competitor defined by $E_{j}, A_{r, j}^{\star}$, and $\tau$ in $B_{2 r}(x)$ for $\star \in\{$ out, in $\}$ as in Lemma 2.8 accordingly, $F_{j}^{\star} \in \mathcal{E}, \Omega \cap \partial F_{j}^{\star}$ is $\mathcal{C}$-spanning $W$,

$$
\begin{gathered}
F_{j}^{\star} \backslash \operatorname{cl}\left(S_{\tau, r}\right)=E_{j} \backslash \operatorname{cl}\left(S_{\tau, r}\right), \\
\lim _{\eta \rightarrow 0^{+}} \mathcal{H}^{n}\left(\left(\partial S_{\tau, r} \cap \partial F_{j}^{\star}\right) \Delta\left(\partial S_{\tau, r} \backslash A_{r, j}^{\star}\right)\right)=0,
\end{gathered}
$$

and

$$
\begin{aligned}
& \limsup _{\eta \rightarrow 0^{+}} \mathcal{H}^{n}\left(S_{\tau, r} \cap \partial F_{j}^{\star}\right) \\
& \quad \leq C(n, \tau) \begin{cases}\mathcal{H}^{n}\left(\partial S_{\tau, r} \backslash\left(A_{r, j}^{\text {out }} \cup E_{j}\right)\right) & \text { if } \star=\text { out } \\
\mathcal{H}^{n}\left(\left(E_{j} \cap \partial S_{\tau, r}\right) \backslash A_{r, j}^{\text {in }}\right) & \text { if } \star=\text { in; }\end{cases}
\end{aligned}
$$


see 2.66, 2.67), 2.68), and 2.69). By 3.11, $\mathcal{H}^{n}\left(\partial S_{\tau, r} \cap \partial E_{j}\right)=0$ and 3.33,

$$
\mathcal{H}^{n}\left(S_{\tau, r} \cap \partial E_{j}\right) \leq \mathcal{H}^{n}\left(\operatorname{cl}\left(S_{\tau, r}\right) \cap \partial F_{j}^{\star}\right)+C_{*} c(n) r^{n+1}+\frac{1}{j} \quad \forall \star \in\{\text { out, in }\} .
$$

By (3.34) and (3.35), taking the limit first as $\eta \rightarrow 0^{+}$and then as $j \rightarrow \infty$, and by also taking into account that $\mu_{j} \stackrel{*}{\rightarrow} \mu$ and that 3.30 holds, we find, in the case $\star=$ out, that

$$
\begin{aligned}
& \mu\left(B_{r}(x)\right) \\
& \leq \limsup _{j \rightarrow \infty} \mathcal{H}^{n}\left(E_{j} \cap \partial S_{\tau, r}\right) \\
& \quad+C(n, \tau) \limsup _{j \rightarrow \infty} \mathcal{H}^{n}\left(\partial S_{\tau, r} \backslash\left(A_{r, j}^{\text {out }} \cup E_{j}\right)\right)+C_{*} c(n) r^{n+1},
\end{aligned}
$$

and, in the case $\star=$ in, that

$$
\begin{aligned}
& \mu\left(B_{r}(x)\right) \\
& \quad \leq \limsup _{j \rightarrow \infty} \mathcal{H}^{n}\left(\partial S_{\tau, r} \backslash E_{j}\right) \\
& \quad+C(n, \tau) \underset{j \rightarrow \infty}{\limsup } \mathcal{H}^{n}\left(\left(E_{j} \cap \partial S_{\tau, r}\right) \backslash A_{r, j}^{\text {in }}\right)+C_{*} c(n) r^{n+1} .
\end{aligned}
$$

We now discuss the cases $x \in \partial^{*} E, x \in K \cap E^{(0)}$, and $x \in K \cap E^{(1)}$ separately.

Case $x \in \partial^{*} E$. We claim that, in this case, for every $\sigma \in(0, \tau)$ and for a.e. $r<r_{0}(\sigma, x)$,

$$
\begin{gathered}
\limsup _{j \rightarrow \infty} \mathcal{H}^{n}\left(\partial S_{\tau, r} \backslash\left(A_{r, j}^{\text {out }} \cup E_{j}\right)\right) \leq C(n) \sigma r^{n}, \\
\limsup _{j \rightarrow \infty}\left|\mathcal{H}^{n}\left(E_{j} \cap \partial S_{\tau, r}\right)-\omega_{n} r^{n}\right| \leq C(n) \tau r^{n} ;
\end{gathered}
$$

see Figure 3.2. We notice that 3.38 and 3.39 combined with 3.36 imply

$$
\frac{\mu\left(B_{r}(x)\right)}{r^{n}} \leq \omega_{n}+C(n) \tau+C(n, \tau) \sigma+C_{*} c(n) r \quad \text { for a.e. } r<r_{0}
$$

which gives $\theta(x) \leq 1$ by letting, in order, $r \rightarrow 0^{+}, \sigma \rightarrow 0^{+}$, and then $\tau \rightarrow 0^{+}$. We now prove 3.38) and 3.39). Since $x \in \partial^{*} E$, we can set $v(x)=v_{E}(x)$. As $v_{E}(x)$ is the outer normal to $E$, by $\partial^{*} E \subset K, 3.29$, and the divergence theorem, we obtain

$$
\left|W_{\tau, \sigma, r_{0}}^{-} \backslash E\right|=\left|W_{\tau, \sigma, r_{0}}^{+} \cap E\right|=0 .
$$

By $\left|W_{\tau, \sigma, r_{0}}^{-} \backslash E\right|=0$, the coarea formula, and Fatou's lemma, we deduce

$$
0=\lim _{j \rightarrow \infty}\left|W_{\tau, \sigma, r_{0}}^{-} \backslash E_{j}\right|=\lim _{j \rightarrow \infty} \int_{0}^{r_{0}} \mathcal{H}^{n}\left(\partial S_{\tau, r} \cap\left(W_{\tau, \sigma, r_{0}}^{-} \backslash E_{j}\right)\right) d r
$$



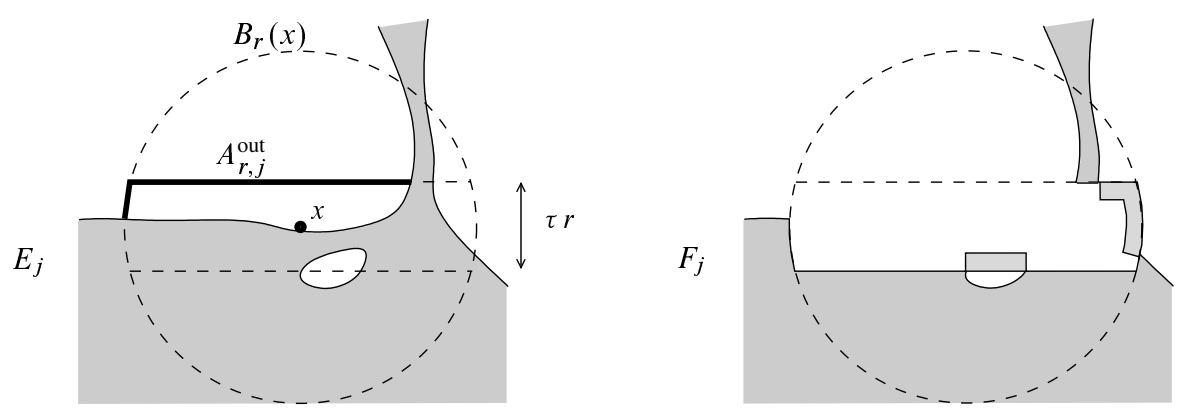

FIGURE 3.2. The slab competitor $F_{j}^{\text {out }}$ is used in proving that $\theta(x) \leq 1$. The fact that $x \in \partial^{*} E$ is used to show that $E_{j} \cap \partial S_{\tau, r}$ consists of a large connected component whose area is close to $\omega_{n} r^{n}$ up to an $\mathrm{o}\left(r^{n}\right)$ error as $r \rightarrow 0^{+}$.

$$
\geq \int_{0}^{r_{0}} \liminf _{j \rightarrow \infty} \mathcal{H}^{n}\left(\Gamma_{\tau, \sigma, r}^{-} \backslash E_{j}\right) d r
$$

and by arguing similarly with $\left|W_{\tau, \sigma, r_{0}}^{+} \cap E\right|=0$, we conclude that, for a.e. $r<r_{0}$,

$$
\begin{gathered}
\lim _{j \rightarrow \infty} \mathcal{H}^{n}\left(\Gamma_{\tau, \sigma, r}^{+} \cap E_{j}\right)=0, \\
\lim _{j \rightarrow \infty} \mathcal{H}^{n}\left(\Gamma_{\tau, \sigma, r}^{-} \backslash E_{j}\right)=0 .
\end{gathered}
$$

By 3.40, 3.41, and since

$$
\partial S_{\tau, r}=\Gamma_{\tau, \sigma, r}^{+} \cup \Gamma_{\tau, \sigma, r}^{-} \cup\left(\partial S_{\tau, r} \cap \partial S_{\sigma, r}\right),
$$

we find that, as $j \rightarrow \infty$,

$$
\begin{aligned}
& \left|\mathcal{H}^{n}\left(\partial S_{\tau, r} \cap E_{j}\right)-\omega_{n} r^{n}\right| \\
& \quad \leq \mathcal{H}^{n}\left(\partial S_{\tau, r} \cap \partial S_{\sigma, r}\right)+\left|\mathcal{H}^{n}\left(\Gamma_{\tau, \sigma, r}^{-} \cap E_{j}\right)-\omega_{n} r^{n}\right|+\mathrm{o}(1) \\
& \quad \leq C(n) \sigma r^{n}+\left|\mathcal{H}^{n}\left(\Gamma_{\tau, \sigma, r}^{-}\right)-\omega_{n} r^{n}\right|+\mathrm{o}(1) \\
& \quad \leq C(n) \tau r^{n}+\mathrm{o}(1),
\end{aligned}
$$

that is, 3.39). At the same time, again by (3.29) and by the coarea formula, assuming without loss of generality that $r_{0}=r_{0}(\sigma, x)$ also satisfies $\mathcal{H}^{n}\left(K \cap \partial B_{r_{0}}(x)\right)=$ 0 in addition to 3.29, we get

$$
\begin{aligned}
0 & =\mu\left(K \cap \operatorname{cl}\left(B_{r_{0}}(x)\right) \backslash V_{\sigma, r_{0}}\right)=\lim _{j \rightarrow \infty} \mathcal{H}^{n}\left(B_{r_{0}}(x) \cap \partial E_{j} \backslash V_{\sigma, r_{0}}\right) \\
& \geq \lim _{j \rightarrow \infty} \mathcal{H}^{n}\left(S_{\tau, r_{0}} \cap \partial E_{j} \backslash V_{\sigma, r_{0}}\right) \\
& \geq \lim _{j \rightarrow \infty} \int_{0}^{r_{0}} \mathcal{H}^{n-1}\left(\partial S_{\tau, r} \cap \partial E_{j} \backslash V_{\sigma, r_{0}}\right) d r
\end{aligned}
$$


that is,

$$
\lim _{j \rightarrow \infty} \mathcal{H}^{n-1}\left(\partial S_{\tau, r} \cap \partial E_{j} \backslash V_{\sigma, r_{0}}\right)=0 \quad \text { for a.e. } r<r_{0} .
$$

Notice that (3.44) implies in particular that

$$
\lim _{j \rightarrow \infty} \mathcal{H}^{n-1}\left(\Gamma_{\tau, \sigma, r}^{+} \cap \partial E_{j}\right)=0 \quad \text { for a.e. } r<r_{0} .
$$

Since $\Gamma_{\tau, \sigma, r}^{+}$is a bi-Lipschitz image of a hemisphere, by Lemma 2.12 .

$$
\mathcal{H}^{n-1}\left(\Gamma_{\tau, \sigma, r}^{+} \cap J\right)^{n /(n-1)} \geq c(n, \tau, \sigma) \mathcal{H}^{n}\left(\Gamma_{\tau, \sigma, r}^{+} \backslash A\right)
$$

whenever $J$ is relatively closed in $\Gamma_{\tau, \sigma, r}^{+}$, and $A$ is an $\mathcal{H}^{n}$-maximal connected component of $\Gamma_{\tau, \sigma, r}^{+} \backslash J$. By (3.45) and (3.46) we find that, if

$$
A_{r, j}^{+} \text {is a maximal } \mathcal{H}^{n} \text {-component of } \Gamma_{\tau, \sigma, r}^{+} \backslash \partial E_{j},
$$

then

$$
\lim _{j \rightarrow \infty} \mathcal{H}^{n}\left(\Gamma_{\tau, \sigma, r}^{+} \backslash A_{r, j}^{+}\right)=0 \quad \text { for a.e. } r<r_{0} .
$$

By connectedness, $A_{r, j}^{+}$is contained in either $A_{r, j}^{\text {out }}, E_{j}$, or

$$
Y_{r, j}=\bigcup\left\{A: A \in \mathcal{A}_{r, j}^{\text {out }} A \neq A_{r, j}^{\text {out }}\right\} .
$$

By combining (3.40) with 3.47) we find that for a.e. $r<r_{0}$, if $j$ is large enough, then

$$
A_{r, j}^{+} \cap E_{j}=\varnothing \text {. }
$$

Similarly, should there be a nonnegligible set of values of $r$ such that for infinitely many values of $j$, the inclusion $A_{r, j}^{+} \subset Y_{r, j}$ holds, then by (3.41) and (3.47), there would be an element of $\mathcal{A}_{r, j}^{\text {out }}$ different from $A_{r, j}^{\text {out }}$ with $\mathcal{H}^{n}$-measure arbitrarily close to $\mathcal{H}^{n}\left(\Gamma_{\tau, \sigma, r}^{+}\right)$; thanks to 3.41, we would then have $\mathcal{H}^{n}\left(A_{r, j}^{\text {out }}\right) \rightarrow 0$, against the $\mathcal{H}^{n}$-maximality of $A_{r, j}^{\text {out }}$ itself. In conclusion, it must be

$$
A_{r, j}^{+} \subset A_{r, j}^{\text {out }} \quad \text { for a.e. } r<r_{0} \text { and for } j \text { large enough. }
$$

By combining (3.48) and (3.47) we conclude that

$$
\lim _{j \rightarrow \infty} \mathcal{H}^{n}\left(\Gamma_{\tau, \sigma, r}^{+} \backslash A_{r, j}^{\text {out }}\right)=0 .
$$

By 3.42, 3.41, and 3.49), we conclude that

$$
\limsup _{j \rightarrow \infty} \mathcal{H}^{n}\left(\partial S_{\tau, r} \backslash\left(A_{r, j}^{\text {out }} \cup E_{j}\right)\right) \leq \mathcal{H}^{n}\left(\partial S_{\tau, r} \cap \partial S_{\sigma, r}\right) \leq C(n) \sigma r^{n},
$$

that is, 3.38). This completes the proof of $\theta(x) \leq 1$ for $x \in \partial^{*} E$. 

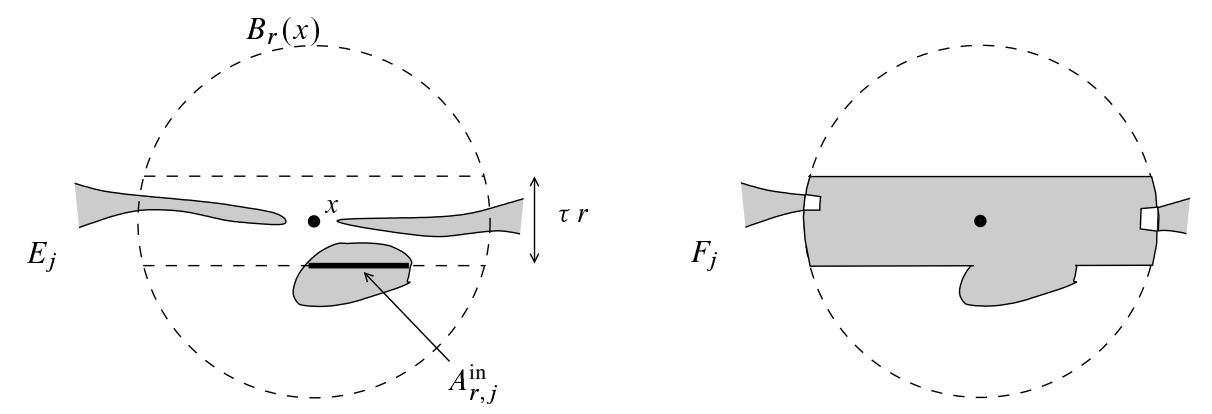

FIGURE 3.3. The slab competitor used in proving that $\theta(x) \leq 2$ when $x \in E^{(0)}$ is the one defined by $A_{r, j}^{\text {in }}$. Since $x \in E^{(0)}$ we can show that $E_{j} \cap \partial S_{\tau, r}$ is o $\left(r^{n}\right)$ as $r \rightarrow 0^{+}$.

Case $x \in E^{(0)}$. We claim that, in this case, for every $\sigma \in(0, \tau)$,

$$
\begin{aligned}
\limsup _{j \rightarrow \infty} \mathcal{H}^{n}\left(E_{j} \cap \partial S_{\tau, r}\right) & \leq C(n) \sigma r^{n}, \\
\limsup _{j \rightarrow \infty}\left|\mathcal{H}^{n}\left(\partial S_{\tau, r} \backslash E_{j}\right)-2 \omega_{n} r^{n}\right| & \leq C(n) \tau r^{n},
\end{aligned}
$$

for a.e. $r<r_{0}(\sigma, x)$; see Figure 3.3. The idea is using the competitor defined by $A_{r, j}^{\text {in }}$ : indeed, 3.50), 3.51, and 3.37) give

$$
\begin{aligned}
\frac{\mu\left(B_{r}(x)\right)}{r^{n}} \leq & \limsup _{j \rightarrow \infty} \frac{\mathcal{H}^{n}\left(\partial S_{\tau, r} \backslash E_{j}\right)}{r^{n}} \\
& +C(n, \tau) \limsup _{j \rightarrow \infty} \frac{\mathcal{H}^{n}\left(\left(E_{j} \cap \partial S_{\tau, r}\right) \backslash A_{r, j}^{\text {in }}\right)}{r^{n}}+C_{*} c(n) r \\
\leq & 2 \omega_{n}+C(n) \tau+C(n, \tau) \sigma+C_{*} c(n) r
\end{aligned}
$$

and then $\theta(x) \leq 2$ by letting, in order, $r \rightarrow 0^{+}, \sigma \rightarrow 0^{+}$, and then $\tau \rightarrow 0^{+}$. The proof of (3.50) and 3.51) is simple: since $x \in E^{(0)}$ and $\partial^{*} E \subset K$, by (3.29) and by the divergence theorem we find that

$$
\left|E \cap B_{r_{0}}(x) \backslash V_{\sigma, r_{0}}\right|=0 .
$$

In particular, by the coarea formula we find that for a.e. $r<r_{0}$,

$$
0=\lim _{j \rightarrow \infty} \mathcal{H}^{n}\left(\left(E_{j} \backslash V_{\sigma, r_{0}}\right) \cap \partial S_{\tau, r}\right)=\lim _{j \rightarrow \infty} \mathcal{H}^{n}\left(E_{j} \cap\left(\Gamma_{\tau, \sigma, r}^{+} \cup \Gamma_{\tau, \sigma, r}^{-}\right)\right)
$$

so that, by 3.42,

$$
\mathcal{H}^{n}\left(E_{j} \cap \partial S_{\tau, r}\right)=\mathcal{H}^{n}\left(\partial S_{\tau, r} \cap \partial S_{\sigma, r}\right)+\mathrm{o}(1) \leq C(n) \sigma r^{n}+\mathrm{o}(1)
$$



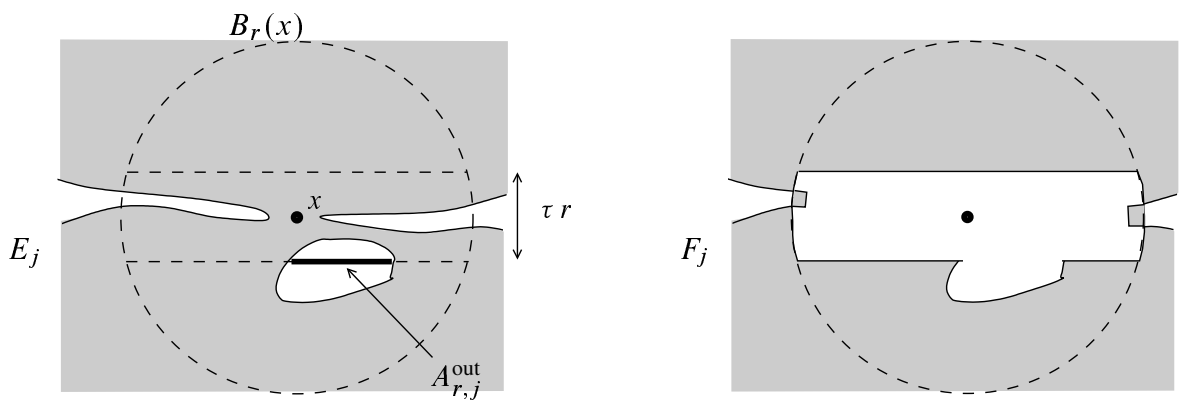

FIGURE 3.4. The slab competitor used in proving that $\theta(x) \leq 2$ when $x \in E^{(1)}$ is the one defined by $A_{r, j}^{\text {out }}$.

as $j \rightarrow \infty$, that is, 3.50 , and

$$
\begin{aligned}
& \left|\mathcal{H}^{n}\left(\partial S_{\tau, r} \backslash E_{j}\right)-2 \omega_{n} r^{n}\right| \\
& \quad \leq \mathcal{H}^{n}\left(\partial S_{\tau, r} \cap \partial S_{\sigma, r}\right)+\left|\mathcal{H}^{n}\left(\Gamma_{\tau, \sigma, r}^{+} \cup \Gamma_{\tau, \sigma, r}^{-}\right)-2 \omega_{n} r^{n}\right|+\mathrm{o}(1) \\
& \quad \leq C(n) \tau r^{n}+\mathrm{o}(1)
\end{aligned}
$$

as $j \rightarrow \infty$, that is, 3.51.

Case $x \in E^{(1)}$. We claim that for every $\sigma \in(0, \tau)$,

$$
\begin{array}{r}
\limsup _{j \rightarrow \infty}\left|\mathcal{H}^{n}\left(E_{j} \cap \partial S_{\tau, r}\right)-2 \omega_{n} r^{n}\right| \leq C(n) \tau r^{n}, \\
\limsup _{j \rightarrow \infty} \mathcal{H}^{n}\left(\partial S_{\tau, r} \backslash E_{j}\right) \leq C(n) \sigma r^{n},
\end{array}
$$

for a.e. $r<r_{0}(\sigma, x)$; see Figure 3.4. Indeed, by using as in the case $x \in \partial^{*} E$ the competitor defined by $A_{r, j}^{\text {out }}$, 3.52) and (3.53) are combined with 3.36) to obtain

$$
\begin{aligned}
\frac{\mu\left(B_{r}(x)\right)}{r^{n}} \leq & \limsup _{j \rightarrow \infty} \frac{\mathcal{H}^{n}\left(E_{j} \cap \partial S_{\tau, r}\right)}{r^{n}} \\
& +C(n, \tau) \limsup _{j \rightarrow \infty} \frac{\mathcal{H}^{n}\left(\partial S_{\tau, r} \backslash\left(A_{r, j}^{\text {out }} \cup E_{j}\right)\right)}{r^{n}}+C_{*} c(n) r \\
\leq & 2 \omega_{n}+C(n) \tau+C(n, \tau) \sigma+C_{*} c(n) r,
\end{aligned}
$$

which gives $\theta(x) \leq 2$ by letting once again $r \rightarrow 0^{+}, \sigma \rightarrow 0^{+}$, and finally $\tau \rightarrow 0^{+}$. To prove (3.52) and 3.53, we notice that by $x \in E^{(1)}, \partial^{*} E \subset K, 3.29$, and the divergence theorem, we have

$$
\left|B_{r_{0}}(x) \backslash\left(V_{\sigma, r_{0}} \cup E\right)\right|=0 .
$$


By the coarea formula, for a.e. $r<r_{0}$ we find

$$
0=\lim _{j \rightarrow \infty} \mathcal{H}^{n}\left(\left(\Gamma_{\tau, \sigma, r}^{+} \cup \Gamma_{\tau, \sigma, r}^{-}\right) \backslash E_{j}\right)
$$

and conclude as in the previous case by exploiting (3.42).

Remark 3.1. We make an important remark on the constructions of Step 5, which will be needed in the proof of Theorem 1.6 . We claim that, under the assumptions on $x$ considered in Step 5, for a.e. $r<r_{0}(\sigma, x)$ we have

$$
\begin{aligned}
& \limsup _{\eta \rightarrow 0^{+}}\left|\mathcal{H}^{n}\left(\left\{y \in \operatorname{cl}\left(S_{\tau, r}\right) \cap \partial F_{j}^{\star}: T_{y}\left(\partial F_{j}^{\star}\right)=T_{x} K\right\}\right)-\theta(x) \omega_{n} r^{n}\right| \\
& \quad \leq C(n) \tau r^{n}+C(n, \tau) \sigma r^{n}+\mathrm{o}(1) \quad \text { as } j \rightarrow \infty .
\end{aligned}
$$

Here $\star=$ out if $x \in \partial^{*} E \cup\left(K \cap E^{(1)}\right), \star=$ in if $x \in K \cap E^{(0)}$, and $\theta(x)=1$ if $x \in \partial^{*} E$ and $\theta(x)=2$ if $x \in K \cap\left(E^{(0)} \cup E^{(1)}\right)$. Consider, for example, the case when $x \in \partial^{*} E$. By (3.34), $\partial S_{\tau, r} \cap \partial F_{j}^{\text {out }} \subset\left(\partial S_{\tau, r} \backslash A_{r, j}^{\text {out }}\right) \cup N_{j}$ with $\lim _{\eta \rightarrow 0^{+}} \mathcal{H}^{n}\left(N_{j}\right)=0$ : thus, by taking into account that

$$
T_{y}\left(\partial F_{j}^{\text {out }}\right)=T_{y}\left(\partial S_{\tau, r}\right) \quad \mathcal{H}^{n} \text {-a.e. on } \partial F_{j}^{\text {out }} \cap \partial S_{\tau, r}
$$

and that

$$
\left\{y \in \partial S_{\tau, r}: T_{y}\left(\partial S_{\tau, r}\right)=T_{x} K\right\}=\partial S_{\tau, r} \backslash \partial B_{r}(x),
$$

(recall that $T_{x} K=v(x)^{\perp}$ ), we have

$$
\begin{aligned}
&\left|\mathcal{H}^{n}\left(\left\{y \in \operatorname{cl}\left(S_{\tau, r}\right) \cap \partial F_{j}^{\text {out }}: T_{y}\left(\partial F_{j}^{\text {out }}\right)=T_{x} K\right\}\right)-\omega_{n} r^{n}\right| \\
& \leq \mid\left|\mathcal{H}^{n}\left(\left\{y \in \partial S_{\tau, r} \cap \partial F_{j}^{\text {out }}: T_{y}\left(\partial F_{j}^{\text {out }}\right)=T_{x} K\right\}\right)-\omega_{n} r^{n}\right| \\
&+\mathcal{H}^{n}\left(S_{\tau, r} \cap \partial F_{j}^{\text {out }}\right) \\
& \leq\left|\mathcal{H}^{n}\left(\left\{y \in \partial S_{\tau, r} \backslash A_{r, j}^{\text {out }}: T_{y}\left(\partial S_{\tau, r}\right)=T_{x} K\right\}\right)-\omega_{n} r^{n}\right| \\
&+\mathcal{H}^{n}\left(N_{j}\right)+\mathcal{H}^{n}\left(S_{\tau, r} \cap \partial F_{j}^{\text {out }}\right) \\
&=\left|\mathcal{H}^{n}\left(\partial S_{\tau, r} \backslash\left(\partial B_{r}(x) \cup A_{r, j}^{\text {out }}\right)\right)-\omega_{n} r^{n}\right|+\mathcal{H}^{n}\left(N_{j}\right)+\mathcal{H}^{n}\left(S_{\tau, r} \cap \partial F_{j}^{\text {out }}\right)
\end{aligned}
$$

so that, by (3.35), 3.38), and $\mathcal{H}^{n}\left(\partial S_{\tau, r} \cap \partial B_{r}(x)\right) \leq C(n) \tau r^{n}$,

$$
\begin{aligned}
& \underset{\eta \rightarrow 0^{+}}{\limsup }\left|\mathcal{H}^{n}\left(\left\{y \in \operatorname{cl}\left(S_{\tau, r}\right) \cap \partial F_{j}^{\text {out }}: T_{y}\left(\partial F_{j}^{\text {out }}\right)=T_{x} K\right\}\right)-\omega_{n} r^{n}\right| \\
& \quad \leq\left|\mathcal{H}^{n}\left(\partial S_{\tau, r} \cap E_{j}\right)-\omega_{n} r^{n}\right|+C(n, \tau) \mathcal{H}^{n}\left(\partial S_{\tau, r} \backslash\left(A_{r, j}^{\text {out }} \cup E_{j}\right)\right)+C(n) \tau r^{n} .
\end{aligned}
$$

By (3.38) and (3.39) we deduce (3.55) when $x \in \partial^{*} E$. The case when $x \in K \cap$ $\left(E^{(0)} \cup E^{(1)}\right)$ is treated analogously and the details are omitted.

Step 6 . We exclude area concentration near $\partial \Omega$ by showing that

$$
\limsup _{\eta \rightarrow 0^{+}} \limsup _{j \rightarrow \infty} \mu_{j}\left(\Omega \cap U_{\eta}(\partial \Omega)\right)=0 .
$$



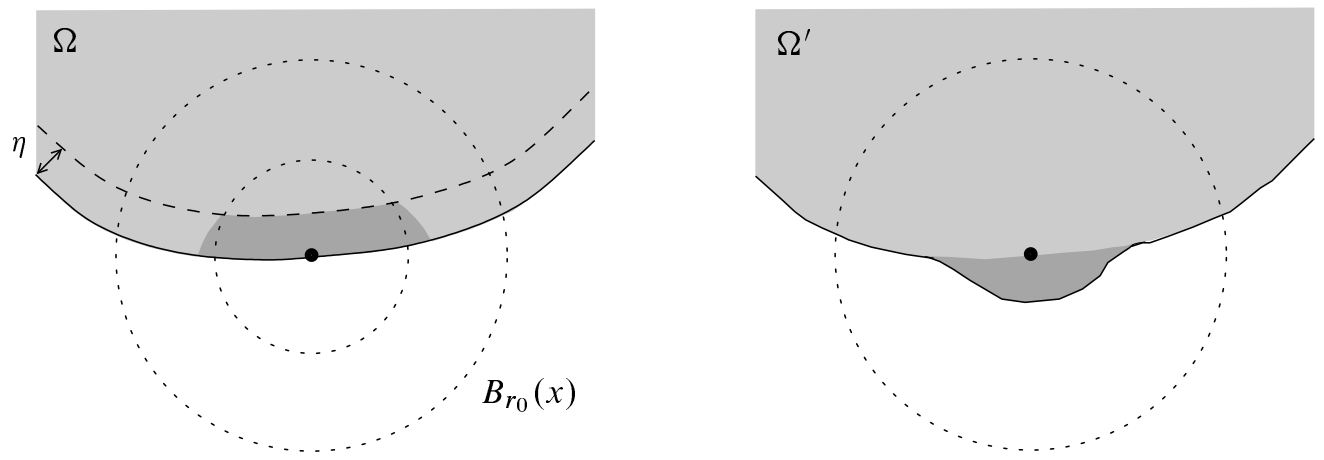

FIGURE 3.5. The boundary diffeomorphism $f$ pushes out $\Omega$ into a larger open set $\Omega^{\prime}$. Regions depicted with the same color are mapped one into the other. Notice that the dark region on the left contains $\Omega \cap U_{\eta}(\partial \Omega) \cap B_{r_{0} / 2}(x)$ and is mapped outside of $\Omega$. The diffeomorphism $f$ can be formally constructed by exploiting the local graphicality of $\Omega$, and the simple details are omitted.

Exploiting the smoothness and boundedness of $\partial \Omega$, we can find $r_{0}>0$ such that Lemma 2.4 holds, and such that for every $x \in \partial \Omega$ there exists an open set $\Omega^{\prime}$ with $\Omega \subset \Omega^{\prime}$ and a homeomorphism $f: \operatorname{cl}(\Omega) \rightarrow \operatorname{cl}\left(\Omega^{\prime}\right)=f(\operatorname{cl}(\Omega))$ with $f(\partial \Omega)=\partial \Omega^{\prime},\{f \neq \mathrm{id}\} \Subset B_{r_{0}}(x)$, and $f\left(B_{r_{0}}(x) \cap \operatorname{cl}(\Omega)\right)=B_{r_{0}}(x) \cap \operatorname{cl}\left(\Omega^{\prime}\right)$, which is a diffeomorphism $f: \Omega \rightarrow \Omega^{\prime}$, and such that

$$
f\left(\Omega \cap U_{\eta}(\partial \Omega) \cap B_{r_{0} / 2}(x)\right) \subset \Omega^{\prime} \backslash \Omega, \quad\|f-\mathrm{id}\|_{C^{1}(\Omega)} \leq C \eta ;
$$

see Figure 3.5. Let $\Omega^{*}=f^{-1}(\Omega)$ and let $F_{j}=f\left(E_{j} \cap \Omega^{*}\right)=f\left(E_{j}\right) \cap \Omega$. Clearly $F_{j} \in \mathcal{E}$, and $f\left(\partial \Omega^{*}\right)=\partial \Omega$ and $\Omega^{*} \cap \partial\left(E_{j} \cap \Omega^{*}\right)=\Omega^{*} \cap \partial E_{j}$ give

$$
\Omega \cap \partial F_{j}=f\left(\Omega^{*}\right) \cap f\left(\partial\left(E_{j} \cap \Omega^{*}\right)\right)=f\left(\Omega^{*} \cap \partial E_{j}\right),
$$

so that $\Omega \cap \partial F_{j}$ is $\mathcal{C}$-spanning $W$ by Lemma 2.4. Assuming without loss of generality that $r_{0}<r_{*}$, by 3.11), $\{f \neq \mathrm{id}\} \Subset B_{r_{0}}(x)$ and $f\left(B_{r_{0}}(x) \cap \operatorname{cl}(\Omega)\right)=$ $B_{r_{0}}(x) \cap \operatorname{cl}\left(\Omega^{\prime}\right)$, we have

$$
\begin{aligned}
& \mathcal{H}^{n}\left(\Omega \cap B_{r_{0}}(x) \cap \partial E_{j}\right) \\
& \quad \leq \mathcal{H}^{n}\left(f\left(B_{r_{0}}(x) \cap \Omega^{*} \cap \partial E_{j}\right)\right)+C_{*}|| F_{j}|-| E_{j}||+\frac{1}{j} \\
& \quad \leq(1+C \eta) \mathcal{H}^{n}\left(B_{r_{0}}(x) \cap \Omega^{*} \cap \partial E_{j}\right)+C_{*}|| F_{j}|-| E_{j}||+\frac{1}{j},
\end{aligned}
$$

where

$$
|| F_{j}|-| E_{j}|| \leq|| E_{j} \cap \Omega^{*}|-| E_{j}||+\int_{E_{j} \cap \Omega^{*}}|J f-1|
$$




$$
\leq\left|\Omega \backslash \Omega^{*}\right|+C \varepsilon \eta \leq C \eta,
$$

so that

$$
\begin{aligned}
\mathcal{H}^{n}\left(\Omega \cap B_{r_{0}}(x) \cap \partial E_{j} \backslash \Omega^{*}\right) & \leq C \eta\left\{\mathcal{H}^{n}\left(\Omega \cap \partial E_{j}\right)+1\right\}+\frac{1}{j} \\
& \leq C \eta\{\psi(\varepsilon)+2\}+\frac{1}{j} .
\end{aligned}
$$

Since $\Omega \cap U_{\eta}(\partial \Omega) \cap B_{r_{0} / 2}(x) \subset \Omega \backslash \Omega^{*}$, by letting $j \rightarrow \infty$ we conclude that

$$
\mu\left(B_{r_{0} / 2}(x) \cap U_{\eta}(\partial \Omega)\right) \leq C \eta \quad \forall x \in \partial \Omega .
$$

By a covering argument we find $\mu\left(\Omega \cap U_{\eta}(\partial \Omega)\right) \leq C \eta$, and thus (3.56) follows.

Step 7. Let us now pick $R>0$ such that $W \cup K \cup E \Subset B_{R}(0)$. If $E_{j} \subset$ $B_{R+1}(0)$ for infinitely many values of $j$, then $|E|=\varepsilon$ and $\mu_{j}\left(\Omega \backslash B_{R+1}(0)\right)=0$, which combined with 3.56 implies $\mu_{j}(\Omega) \rightarrow \mu(\Omega)=\mathcal{F}(K, E)$ as $j \rightarrow \infty$, and thus $\psi(\varepsilon)=\mathcal{F}(K, E)$ with $(K, E) \in \mathcal{K}$ and $|E|=\varepsilon$ : thus $(K, E)$ is a generalized minimizer of $\psi(\varepsilon)$, as desired. We now assume without loss of generality that $\left|E_{j} \backslash B_{R+1}(0)\right|>0$ for every $j$. By (3.5),

$$
\begin{aligned}
& \limsup _{j \rightarrow \infty}\left|E_{j} \cap\left(B_{R+1}(0) \backslash B_{R}(0)\right)\right| \\
& \quad=\limsup _{j \rightarrow \infty} \mathcal{H}^{n}\left(\left(B_{R+1}(0) \backslash B_{R}(0)\right) \cap \partial E_{j}\right)=0 .
\end{aligned}
$$

By the coarea formula, this implies that for a.e. $s \in(R, R+1)$,

$$
\limsup _{j \rightarrow \infty} \mathcal{H}^{n}\left(E_{j} \cap \partial B_{s}(0)\right)=\limsup _{j \rightarrow \infty} \mathcal{H}^{n-1}\left(\partial E_{j} \cap \partial B_{s}(0)\right)=0 .
$$

We fix a value of $s$ such that (3.58) holds, and we let $A_{j}$ denote an $\mathcal{H}^{n}$-maximal connected component of $\partial B_{S}(0) \backslash \partial E_{j}$. It must be $A_{j} \cap E_{j}=\varnothing$ : otherwise, by the spherical isoperimetric inequality, $A_{j} \subset E_{j}$ would imply

$$
\begin{aligned}
C(n) \mathcal{H}^{n-1}\left(\partial B_{S}(0) \cap \partial E_{j}\right)^{n /(n-1)} & \geq \mathcal{H}^{n}\left(\partial B_{S}(0) \backslash A_{j}\right) \geq \mathcal{H}^{n}\left(\partial B_{S}(0) \backslash E_{j}\right) \\
& \geq c(n) R^{n}-\mathcal{H}^{n}\left(E_{j} \cap \partial B_{S}(0)\right),
\end{aligned}
$$

a contradiction to (3.58). Since $A_{j} \cap E_{j}=\varnothing$, we can consider the exterior cup competitor defined by $E_{j}$ and $A_{j}$. More precisely, for every $j$ there exists a decreasing sequence $\left\{\eta_{k}^{j}\right\}_{k=1}^{\infty}$ with $\lim _{k \rightarrow \infty} \eta_{k}^{j}=0$ such that, setting

$$
\begin{gathered}
Y_{j}=\partial B_{S}(0) \backslash \operatorname{cl}\left(\left(E_{j} \cap \partial B_{s}(0)\right) \cup A_{j}\right), \\
S_{j}=\partial E_{j} \cap \operatorname{cl}\left(A_{j}\right) \backslash\left(\operatorname{cl}\left(\left(E_{j} \cap \partial B_{s}(0)\right) \cup Y_{j}\right)\right), \\
U_{j, k}=\partial B_{S}(0) \cap\left\{\mathrm{d}_{S_{j}}<\eta_{k}^{j}\right\}, \quad Z_{j, k}=Y_{j} \cup\left(U_{j, k} \backslash \operatorname{cl}\left(E_{j} \cap \partial B_{S}(0)\right)\right),
\end{gathered}
$$

the sets

$$
F_{j, k}=\left(E_{j} \cap B_{s}(0)\right) \cup M_{\eta_{k}^{j}}\left(Z_{j, k}\right)
$$


satisfy $F_{j, k} \in \mathcal{E}$, with $\Omega \cap \partial F_{j, k} \mathcal{C}$-spanning $W, F_{j, k} \subset B_{R+1}$, and

$$
\begin{aligned}
\limsup _{k} & \mathcal{H}^{n}\left(\Omega \cap \partial F_{j, k}\right) \\
& \leq \mathcal{H}^{n}\left(\Omega \cap B_{S}(0) \cap \partial E_{j}\right)+2 \mathcal{H}^{n}\left(\partial B_{S}(0) \backslash A_{j}\right) \\
& \leq \mathcal{H}^{n}\left(\Omega \cap B_{S}(0) \cap \partial E_{j}\right)+C(n) \mathcal{H}^{n-1}\left(\partial B_{S}(0) \cap \partial E_{j}\right)^{n /(n-1)} .
\end{aligned}
$$

Since $\left|E_{j} \backslash B_{R+1}(0)\right|>0$ for every $j$, we can select $k(j)$ sufficiently large so that

$$
\begin{aligned}
\mathcal{H}^{n}\left(\Omega \cap \partial F_{j, k(j)}\right) \leq & \mathcal{H}^{n}\left(\Omega \cap B_{S}(0) \cap \partial E_{j}\right) \\
& +C(n) \mathcal{H}^{n-1}\left(\partial B_{S}(0) \cap \partial E_{j}\right)^{n /(n-1)}+\frac{1}{j},
\end{aligned}
$$

as well as $\left|E_{j} \backslash B_{S}(0)\right|>\left|M_{\eta_{k(j)}^{j}}\left(Z_{j, k(j)}\right)\right|$; then, after setting $F_{j}=F_{j, k(j)}$, define $\rho_{j}>0$ by the equation

$$
\left|B_{\rho_{j}}\right|=\left|E_{j}\right|-\left|F_{j}\right|=\left|E_{j} \backslash B_{S}(0)\right|-\left|M_{\eta_{k(j)}^{j}}\left(Z_{j, k(j)}\right)\right| .
$$

In particular, $\left|B_{\rho_{j}}\right| \leq \varepsilon$, so that we can find $x \in \Omega$ such that $\operatorname{cl}\left(B_{\rho_{j}}(x)\right) \cap \operatorname{cl}\left(F_{j}\right)=$ $\varnothing$ and

$$
E_{j}^{*}=F_{j} \cup B_{\rho_{j}}(x) \subset B_{R+1+C(n) \varepsilon^{1 /(n+1)}}(0) \quad \forall j .
$$

We notice that $E_{j}^{*} \in \mathcal{E}$ with $\left|E_{j}^{*}\right|=\varepsilon$ and $\Omega \cap \partial F_{j} \subset \Omega \cap \partial E_{j}^{*}$, so that $\Omega \cap \partial E_{j}^{*}$ is $\mathcal{C}$ spanning $W$ : in particular, $\psi(\varepsilon) \leq \mathcal{H}^{n}\left(\Omega \cap \partial E_{j}^{*}\right)$. By the Euclidean isoperimetric inequality, and since $\left|B_{\rho_{j}}\right| \leq\left|E_{j} \backslash B_{S}(0)\right|$ by definition of $\rho_{j}$, we have

$$
P\left(B_{\rho_{j}}\right) \leq P\left(E_{j} \backslash B_{S}(0)\right)=\mathcal{H}^{n}\left(\partial E_{j} \backslash B_{S}(0)\right)+\mathcal{H}^{n}\left(E_{j} \cap \partial B_{S}(0)\right),
$$

so that by (3.58) and (3.60) we get

$$
\begin{aligned}
\psi(\varepsilon) & \leq \limsup _{j \rightarrow \infty} \mathcal{H}^{n}\left(\Omega \cap \partial E_{j}^{*}\right) \leq \limsup _{j \rightarrow \infty} \mathcal{H}^{n}\left(\Omega \cap \partial F_{j}\right)+P\left(B_{\rho_{j}}\right) \\
& \leq \limsup _{j \rightarrow \infty} \mathcal{H}^{n}\left(\Omega \cap \partial E_{j}\right)+2 C(n) \limsup _{j \rightarrow \infty} \mathcal{H}^{n-1}\left(\partial B_{S}(0) \cap \partial E_{j}\right)^{n /(n-1)} \\
& =\psi(\varepsilon) .
\end{aligned}
$$

We have thus proved that $\left\{E_{j}^{*}\right\}_{j}$ is a minimizing sequence for $\psi(\varepsilon)$, with $E_{j}^{*} \subset$ $B_{R^{*}}(0)$ for some $R^{*}$ depending only on $R, n$, and $\varepsilon$. By repeating the argument of the first six steps with $E_{j}^{*}$ in place of $E_{j}$, we see that $E_{j}^{*} \rightarrow E^{*}$ in $L^{1}(\Omega)$ and $\mu_{j}^{*}=\mathcal{H}^{n}\left\llcorner\left(\Omega \cap \partial E_{j}^{*}\right) \stackrel{*}{\rightarrow} \mu^{*}\right.$ where $\mu^{*}=2 \mathcal{H}^{n}\left\llcorner\left(K^{*} \backslash \partial^{*} E^{*}\right)+\mathcal{H}^{n}\left\llcorner\partial^{*} E^{*}\right.\right.$, and where $\left(K^{*}, E^{*}\right) \in \mathcal{K}$ with $\left|E^{*}\right|=\varepsilon$ and with

$$
\limsup _{\eta \rightarrow 0^{+}} \limsup _{j \rightarrow \infty} \mu_{j}^{*}\left(\Omega \cap U_{\eta}(\partial \Omega)\right)=0 .
$$


Therefore $\mu_{j}^{*}(\Omega) \rightarrow \mu^{*}(\Omega)=\mathcal{F}\left(K^{*}, E^{*}\right)$ and in conclusion

$$
\mathcal{F}\left(K^{*}, E^{*}\right)=\mu^{*}(\Omega)=\lim _{j \rightarrow \infty} \mu_{j}^{*}(\Omega)=\psi(\varepsilon)
$$

so that, by $\left|E^{*}\right|=\varepsilon,\left(K^{*}, E^{*}\right)$ is indeed a generalized minimizer of $\psi(\varepsilon)$. This concludes the proof of the theorem.

\section{The Euler-Lagrange Equation: Proof of Theorem 1.6}

Proof of THEOREM 1.6. Let $(K, E)$ be a generalized minimizer of $\psi(\varepsilon)$ and $f: \Omega \rightarrow \Omega$ be a diffeomorphism such that $|f(E)|=|E|$. We want to prove that

$$
\mathcal{F}(K, E) \leq \mathcal{F}(f(K), f(E))
$$

Let $K^{\prime}$ denote the set of points of approximate differentiability of $K$, so that $\mathcal{H}^{n}\left(K \backslash K^{\prime}\right)=0$, and for $x \in K^{\prime}$ denote by $T_{x}=T_{x} K=v_{x}^{\perp}$ the approximate tangent plane to $K$ at $x$, where $v_{x} \in \mathbb{S}^{n}$ is chosen so that $v_{x}=v_{E}(x)$ if $x \in \partial^{*} E$. As in Step 5 of the proof of Theorem 1.4, for every $\sigma>0$ we introduce $r_{0}=r_{0}(\sigma, x)$ such that

$$
K \cap B_{r}(x) \subset S_{\sigma, r}^{x}=\left\{y \in B_{r}(x):\left|(y-x) \cdot v_{x}\right|<\sigma r\right\} \quad \forall r<r_{0}(\sigma, x) ;
$$

see (3.29). In fact, by Egoroff's theorem, we can find a compact set $K^{*} \subset K^{\prime}$ with $\mathcal{H}^{n}\left(K \backslash K^{*}\right)<\sigma$ such that $r_{*}(\sigma)=\max \left\{r_{0}(\sigma, x): x \in K^{*}\right\} \rightarrow 0^{+}$as $\sigma \rightarrow 0^{+}$, that is, such that (4.2) holds uniformly on $K^{*}$,

$$
K \cap B_{r}(x) \subset S_{\sigma, r}^{x} \quad \forall x \in K^{*} \forall r<r_{*}(\sigma) .
$$

Similarly, if $G_{n}$ denotes the family of the $n$-planes in $\mathbb{R}^{n+1}$, endowed with a distance $d$, by Lusin's theorem and up to further decreasing the size of $K^{*}$ while keeping $\mathcal{H}^{n}\left(K \backslash K^{*}\right)<\sigma$, we can make sure that

$$
\sup _{x, y \in K^{*}|x-y|<r} d\left(T_{x}, T_{y}\right)+\sup _{x, y \in K^{*}|y-x|<r}|\nabla f(x)-\nabla f(y)| \leq \omega_{*}(r)
$$

for a function $\omega_{*}(r) \rightarrow 0^{+}$as $r \rightarrow 0^{+}$. Finally, since

$$
\begin{aligned}
& \left\{\begin{array}{l}
\mathcal{H}^{n}\left(B_{r}(x) \cap \partial^{*} E\right)=\mathrm{o}\left(r^{n}\right), \\
\mathcal{H}^{n}\left(B_{r}(x) \cap\left(K \backslash \partial^{*} E\right)\right)=\omega_{n} r^{n}+\mathrm{o}\left(r^{n}\right) \quad \text { for } \mathcal{H}^{n} \text {-a.e. } x \in K \backslash \partial^{*} E,
\end{array}\right. \\
& \left\{\begin{array}{l}
\mathcal{H}^{n}\left(B_{r}(x) \cap \partial^{*} E\right)=\omega_{n} r^{n}+\mathrm{o}\left(r^{n}\right) \\
\mathcal{H}^{n}\left(B_{r}(x) \cap\left(K \backslash \partial^{*} E\right)\right)=\mathrm{o}\left(r^{n}\right) \text { for } \mathcal{H}^{n} \text {-a.e. } x \in \partial^{*} E
\end{array}\right.
\end{aligned}
$$

as $r \rightarrow 0^{+}$, by Egoroff's theorem, up to decreasing $K^{*}$ and increasing $\omega_{*}$, we can also obtain

$$
\begin{aligned}
& \sup _{x \in K^{*} \backslash \partial^{*} E} \mathcal{H}^{n}\left(B_{r}(x) \cap \partial^{*} E\right) \\
& \quad+\left|\mathcal{H}^{n}\left(B_{r}(x) \cap\left(K \backslash \partial^{*} E\right)\right)-\omega_{n} r^{n}\right| \leq \omega_{*}(r) r^{n},
\end{aligned}
$$




$$
\begin{aligned}
& \sup _{x \in K^{*} \cap \partial^{*} E}\left|\mathcal{H}^{n}\left(B_{r}(x) \cap \partial^{*} E\right)-\omega_{n} r^{n}\right| \\
& \quad+\mathcal{H}^{n}\left(B_{r}(x) \cap\left(K \backslash \partial^{*} E\right)\right) \leq \omega_{*}(r) r^{n},
\end{aligned}
$$

while still keeping $\mathcal{H}^{n}\left(K \backslash K^{*}\right)<\sigma$ and $\omega_{*}(r) \rightarrow 0^{+}$as $r \rightarrow 0^{+}$.

Let $\left\{E_{j}\right\}_{j}$ be a minimizing sequence for $\psi(\varepsilon)$ converging to $(K, E)$ as in (1.13), and consider a point $x \in K^{*}$. Given $\tau \in(0,1)$ and $\sigma \in(0, \tau)$, for a.e. $r<r_{*}(\sigma)$ such that $B_{2 r}(x) \Subset \Omega$, we have that $\partial S_{\tau, r}^{x} \cap \partial E_{j}$ is $\mathcal{H}^{n-1}$-rectifiable for every $j$ (with the exceptional set depending on $x$ ). For such values of $r$ and for every $\eta \in(0, r / 2)$, we can set

$$
F_{j}^{x}= \begin{cases}F_{j}^{\text {out }} & \text { if } x \in \partial^{*} E \cup\left(K^{*} \cap E^{(1)}\right), \\ F_{j}^{\text {in }} & \text { if } x \in K^{*} \cap E^{(0)},\end{cases}
$$

with $F_{j}^{\text {out }}$ and $F_{j}^{\text {in }}$ defined as in Step 5 of the proof of Theorem 1.4. In particular, $F_{j}^{x} \in \mathcal{E}, \Omega \cap \partial F_{j}^{x}$ is $\mathcal{C}$-spanning $W, F_{j}^{x} \backslash \operatorname{cl}\left(S_{\tau, r}^{x}\right)=E_{j} \backslash \operatorname{cl}\left(S_{\tau, r}^{x}\right)$, and, as proved in 3.55), for a.e. $r<r_{*}(\sigma)$ we have

$$
\begin{aligned}
& \limsup _{\eta \rightarrow 0^{+}}\left|\mathcal{H}^{n}\left(\left\{y \in \operatorname{cl}\left(S_{\tau, r}^{x}\right) \cap \partial F_{j}^{x}: T_{y}\left(\partial F_{j}^{x}\right)=T_{x}\right\}\right)-\theta(x) \omega_{n} r^{n}\right| \\
& \quad \leq C(n) \tau r^{n}+C(n, \tau) \sigma r^{n}+\mathrm{o}(1) \quad \text { as } j \rightarrow \infty,
\end{aligned}
$$

where $\theta(x)=1$ if $x \in \partial^{*} E$ and $\theta(x)=2$ if $x \in K \cap\left(E^{(0)} \cup E^{(1)}\right)$, as well as

$$
\limsup _{j \rightarrow \infty} \limsup _{\eta \rightarrow 0^{+}} \mathcal{H}^{n}\left(S_{\tau, r}^{x} \cap \partial F_{j}^{x}\right) \leq C(n, \tau) \sigma r^{n}
$$

see (3.35), (3.38), 3.50), and 3.53). By Besicovitch-Vitali's covering theorem and by Federer's theorem 2.1, we can find a finite disjoint family of closed balls $\left\{B_{i}=\operatorname{cl}\left(B_{r_{i}}\left(x_{i}\right)\right)\right\}_{i}$ such that $B_{i} \Subset \Omega$ and

$$
\begin{gathered}
\mathcal{H}^{n}\left(K^{*} \backslash \bigcup B_{r_{i}}\left(x_{i}\right)\right)<\sigma, \\
x_{i} \in K^{*} \cap\left(E^{(0)} \cup E^{(1)} \cup \partial^{*} E\right), \quad r_{i}<r_{*}(\sigma) .
\end{gathered}
$$

We let $\eta<\min _{i}\left\{r_{i} / 2\right\}$, define $F_{j}^{x_{i}}$ accordingly, and set

$$
S_{i}=S_{\tau, r_{i}}^{x_{i}} \Subset B_{i}, \quad T_{i}=T_{x_{i}}, \quad F_{j}^{i}=F_{j}^{x_{i}} .
$$

Correspondingly, we define a sequence $\left\{F_{j}\right\}_{j} \subset \mathcal{E}$ with $\Omega \cap \partial F_{j} \mathcal{C}$-spanning $W$ by setting

$$
F_{j} \backslash \bigcup_{i} B_{i}=E_{j} \backslash \bigcup_{i} B_{i}, \quad F_{j} \cap B_{i}=F_{j}^{i} \cap B_{i} .
$$

Since $F_{j}^{i} \backslash \operatorname{cl}\left(S_{i}\right)=E_{j} \backslash \operatorname{cl}\left(S_{i}\right)$ we find that

$$
F_{j} \backslash \bigcup_{i} \operatorname{cl}\left(S_{i}\right)=E_{j} \backslash \bigcup_{i} \operatorname{cl}\left(S_{i}\right)
$$


and, setting

$$
\theta_{i}=1 \quad \text { if } x_{i} \in \partial^{*} E, \quad \theta_{i}=2 \quad \text { if } x_{i} \in E^{(0)} \cup E^{(1)},
$$

we deduce from (4.7) and (4.8) that, for each $i$,

$$
\begin{aligned}
& \limsup _{\eta \rightarrow 0^{+}}\left|\mathcal{H}^{n}\left(\left\{y \in \operatorname{cl}\left(S_{i}\right) \cap \partial F_{j}: T_{y}\left(\partial F_{j}\right)=T_{i}\right\}\right)-\theta_{i} \omega_{n} r_{i}^{n}\right| \\
& \leq C(n) \tau r_{i}^{n}+C(n, \tau) \sigma r_{i}^{n}+\mathrm{o}(1), \\
& \quad \limsup _{\eta \rightarrow 0^{+}} \mathcal{H}^{n}\left(S_{i} \cap \partial F_{j}\right) \leq C(n, \tau) \sigma r_{i}^{n}+\mathrm{o}(1),
\end{aligned}
$$

as $j \rightarrow \infty$. Now let $C_{*}$ and $\varepsilon_{*}$ be the volume-fixing variation constants defined by $f(E)$. By the monotonicity formula (3.27), which can be applied to $B_{r_{i}}\left(x_{i}\right)$ as $x_{i} \in K$, we have

$$
e^{-\Lambda r_{*}(\sigma)} \theta_{i} \omega_{n} r_{i}^{n} \leq e^{-\Lambda r_{i}} \theta_{i} \omega_{n} r_{i}^{n} \leq \mu\left(B_{r_{i}}\left(x_{i}\right)\right)=\mu\left(S_{i}\right)
$$

where in the last identity we have used (4.3), and where $\Lambda$ depends on $E$. By (4.15), $\theta_{i} \geq 1$, and $\mu=\theta \mathcal{H}^{n}\llcorner K$ with $\theta \leq 2$,

$$
\sum_{i} r_{i}^{n} \leq C(n, E) \sum_{i} \mathcal{H}^{n}\left(K \cap B_{i}\right) \leq C(n, E) \mathcal{H}^{n}(K)=C(n, E, K)
$$

so that, by 4.11), $\left|S_{i}\right| \leq C(n) \tau r_{i}^{n+1}$, and $r_{i} \leq r_{*}(\sigma) \leq 1$, we find

$$
\left|F_{j} \Delta E_{j}\right| \leq \sum_{i}\left|S_{i}\right| \leq C(n, E, K) \tau
$$

Therefore,

$$
\left|f\left(F_{j}\right) \Delta f(E)\right| \leq C\left(n, E, \operatorname{Lip}(f), \mathcal{H}^{n}(K)\right)\left\{\tau+\left|E_{j} \Delta E\right|\right\}<\varepsilon_{*}
$$

provided $j$ is large enough and $\tau$ is small enough depending on $\varepsilon_{*}$. By the volumefixing variations construction, for each $j$ large enough there exists a smooth map $\Phi_{j}:\left(-\varepsilon_{*}, \varepsilon_{*}\right) \times \mathbb{R}^{n+1} \rightarrow \mathbb{R}^{n+1}$ such that, for every $|v|<\varepsilon_{*}, \Phi_{j}(v, \cdot)$ is a diffeomorphism with $\Phi_{j}(v, \Omega)=\Omega$ and

$$
\left|\Phi_{j}\left(v, f\left(F_{j}\right)\right)\right|=v+\left|f\left(F_{j}\right)\right|, \quad \mathcal{H}^{n}\left(\Phi_{j}(v, \Sigma)\right) \leq \mathcal{H}^{n}(\Sigma)+C_{*}|v| \mathcal{H}^{n}(\Sigma),
$$

for every $\mathcal{H}^{n}$-rectifiable set $\Sigma \subset \Omega$. In particular, if we set

$$
G_{j}=\Phi_{j}\left(v_{j}, f\left(F_{j}\right)\right), \quad v_{j}=|f(E)|-\left|f\left(F_{j}\right)\right|=|E|-\left|f\left(F_{j}\right)\right|,
$$

then we find that $G_{j} \in \mathcal{E},\left|G_{j}\right|=|E|=\varepsilon$, and

$\mathcal{H}^{n}\left(\Omega \cap \partial G_{j}\right) \leq\left(1+C\left(n, E, \operatorname{Lip}(f), \mathcal{H}^{n}(K)\right)\left\{\tau+\left|E_{j} \Delta E\right|\right\}\right) \mathcal{H}^{n}\left(\Omega \cap \partial f\left(F_{j}\right)\right)$.

Since $\Omega \cap \partial F_{j}$ is $\mathcal{C}$-spanning $W$, so is $\Omega \cap \partial G_{j}$ thanks to Lemma 2.3 , so that the minimizing sequence property of $E_{j}$ implies

$$
\mathcal{H}^{n}\left(\Omega \cap \partial E_{j}\right) \leq\left(1+C\left\{\tau+\left|E_{j} \Delta E\right|\right\}\right) \mathcal{H}^{n}\left(\Omega \cap \partial f\left(F_{j}\right)\right)+\frac{1}{j}
$$


where, here and for the rest of the proof, $C$ is a generic constant depending on $K$, $E, f$, and $n$. We now claim that

$$
\underset{\sigma \rightarrow 0^{+}}{\limsup } \limsup _{j \rightarrow \infty} \limsup _{\eta \rightarrow 0^{+}} \mathcal{H}^{n}\left(\Omega \cap \partial f\left(F_{j}\right)\right) \leq \mathcal{F}(f(K), \quad f(E))+C \tau
$$

Notice that by combining (4.17) and 4.18, and by finally letting $\tau \rightarrow 0^{+}$, we complete the proof of 4.1 .

To prove (4.18), we notice that $f(\Omega)=\Omega, \Omega \cap \partial f\left(F_{j}\right)=f\left(\Omega \cap \partial F_{j}\right)$, and (4.11) yield

$$
\begin{aligned}
\mathcal{H}^{n} & \left(\Omega \cap \partial f\left(F_{j}\right)\right) \\
& \leq \mathcal{H}^{n}\left(f\left(\Omega \cap \partial E_{j} \backslash \bigcup_{i} \operatorname{cl}\left(S_{i}\right)\right)\right)+\sum_{i} \int_{\operatorname{cl}\left(S_{i}\right) \cap \partial F_{j}} J^{\partial F_{j}} f d \mathcal{H}^{n}
\end{aligned}
$$

where

$$
\limsup _{j \rightarrow \infty} \limsup _{\eta \rightarrow 0^{+}} \stackrel{n}{\mathcal{H}}\left(f\left(\Omega \cap \partial E_{j} \backslash \bigcup_{i} \operatorname{cl}\left(S_{i}\right)\right)\right) \leq C \mathcal{H}^{n}\left(K \backslash \bigcup_{i} S_{i}\right) \leq C \sigma
$$

by (4.3), 4.9), and $\mathcal{H}^{n}\left(K \backslash K^{*}\right)<\sigma$. Hence, as

$$
\mathcal{H}^{n}\left(\Omega \cap \partial f\left(F_{j}\right)\right) \leq \sum_{i} \int_{\operatorname{cl}\left(S_{i}\right) \cap \partial F_{j}} J^{\partial F_{j}} f d \mathcal{H}^{n}+C \sigma+\mathrm{o}(1)
$$

where o(1) $\rightarrow 0^{+}$if we let first $\eta \rightarrow 0^{+}$and then $j \rightarrow \infty$.

If we set

$$
Z_{i}=\left\{y \in \partial S_{i} \cap \partial F_{j}: T_{y}\left(\partial F_{j}\right)=T_{i}\right\}
$$

then by (4.13) and (4.14) we find

$$
\begin{aligned}
\mathcal{H}^{n}\left(\left(\operatorname{cl}\left(S_{i}\right) \cap \partial F_{j}\right) \Delta Z_{i}\right) & \leq C(n) \tau r_{i}^{n}+C(n, \tau) \sigma r_{i}^{n}+\mathrm{o}(1) \\
\left|\mathcal{H}^{n}\left(Z_{i}\right)-\theta_{i} \omega_{n} r_{i}^{n}\right| & \leq C(n) \tau r_{i}^{n}+C(n, \tau) \sigma r_{i}^{n}+\mathrm{o}(1)
\end{aligned}
$$

where o(1) $\rightarrow 0^{+}$if we let first $\eta \rightarrow 0^{+}$and then $j \rightarrow \infty$. Also, it follows from (4.15), the characterization of $\mu$, and (4.6) that

$$
e^{-\Lambda r_{*}(\sigma)} \theta_{i} \omega_{n} r_{i}^{n} \leq \theta_{i} \mathcal{H}^{n}\left(S_{i} \cap K\right)+\omega_{*}\left(r_{i}\right) r_{i}^{n}
$$


By (4.4), 4.20), and $r_{i}<r_{*}(\sigma)$, we thus find

$$
\begin{aligned}
& \int_{\mathrm{cl}\left(S_{i}\right) \cap \partial F_{j}} J^{\partial F_{j}} f \\
& \leq \int_{Z_{i}} J^{T_{i}} f+(\operatorname{Lip} f)^{n}\{C(n) \tau+C(n, \tau) \sigma\} r_{i}^{n}+\mathrm{o}(1) \\
& \leq \theta_{i} \omega_{n} r_{i}^{n}\left\{J^{T_{i}} f\left(x_{i}\right)+C(n) \omega_{*}\left(r_{i}\right)\right\}+C\{\tau+C(n, \tau) \sigma\} r_{i}^{n}+\mathrm{o}(1) \\
& \leq\left\{J^{T_{i}} f\left(x_{i}\right)+C\left(\omega_{*}\left(r_{*}(\sigma)\right)+\tau+C(n, \tau) \sigma\right)\right\} \\
& \times\left(\theta_{i} \mathcal{H}^{n}\left(S_{i} \cap K\right)+\omega_{*}\left(r_{*}(\sigma)\right) r_{i}^{n}\right) e^{\Lambda r_{*}(\sigma)}+\mathrm{o}(1) \\
&= J^{T_{i}} f\left(x_{i}\right)\left(\theta_{i} \mathcal{H}^{n}\left(S_{i} \cap K^{*}\right)+\alpha_{i}+\omega_{*}\left(r_{*}(\sigma)\right) r_{i}^{n}\right) e^{\Lambda r_{*}(\sigma)} \\
&+C\left\{\omega_{*}\left(r_{*}(\sigma)\right)+\tau+C(n, \tau) \sigma\right\}\left(\mathcal{H}^{n}\left(S_{i} \cap K\right)+\omega_{*}\left(r_{*}(\sigma)\right) r_{i}^{n}\right) e^{\Lambda r_{*}(\sigma)} \\
&+\mathrm{o}(1)
\end{aligned}
$$

where we have set

$$
\alpha_{i}=\theta_{i} \mathcal{H}^{n}\left(S_{i} \cap\left(K \backslash K^{*}\right)\right) \quad \text { so that } \sum_{i} \alpha_{i}<2 \sigma .
$$

Now, again by (4.4) we see that

$$
\begin{aligned}
\theta_{i} J^{T_{i}} f\left(x_{i}\right) \mathcal{H}^{n}\left(S_{i} \cap K^{*}\right) & \leq \theta_{i} \int_{S_{i} \cap K^{*}} J^{K} f d \mathcal{H}^{n}+C(n) \omega_{*}\left(r_{i}\right) \mathcal{H}^{n}\left(S_{i} \cap K^{*}\right) \\
& =\theta_{i} 2 \mathcal{H}^{n}\left(f\left(S_{i} \cap K^{*}\right)\right)+C(n) \omega_{*}\left(r_{i}\right) \mathcal{H}^{n}\left(S_{i} \cap K^{*}\right) .
\end{aligned}
$$

By combining this last relation with (4.16), 4.19), 4.21), and $r_{i}<r_{*}(\sigma)$, we find that

$$
\begin{aligned}
\mathcal{H}^{n}\left(\Omega \cap \partial f\left(F_{j}\right)\right) \leq & e^{\Lambda r_{*}(\sigma)} \sum_{i} \theta_{i} \mathcal{H}^{n}\left(f\left(S_{i} \cap K^{*}\right)\right) \\
& +C\left\{\omega_{*}\left(r_{*}(\sigma)\right)+\tau+C(n, \tau) \sigma\right\} e^{\Lambda r_{*}(\sigma)}+\mathrm{o}(1)
\end{aligned}
$$

with o(1) $\rightarrow 0$ as first $\eta \rightarrow 0^{+}$and then $j \rightarrow \infty$. If $x_{i} \in K^{*} \backslash \partial^{*} E$, then $\theta_{i}=2$ and by $(4.5)$ we have

$$
\begin{aligned}
\theta_{i} \mathcal{H}^{n}\left(f\left(S_{i} \cap K^{*}\right)\right) & \leq 2 \mathcal{H}^{n}\left(f\left(S_{i} \cap\left(K^{*} \backslash \partial^{*} E\right)\right)\right)+2 \operatorname{Lip}(f)^{n} \omega_{*}\left(r_{i}\right) r_{i}^{n} \\
& \leq 2 \mathcal{H}^{n}\left(f\left(S_{i} \cap\left(K \backslash \partial^{*} E\right)\right)\right)+C \omega_{*}\left(r_{*}(\sigma)\right) r_{i}^{n} ;
\end{aligned}
$$

if, instead, $x_{i} \in \partial^{*} E$, then $\theta_{i}=1$ and (4.6) give

$$
\begin{aligned}
\theta_{i} \mathcal{H}^{n}\left(f\left(S_{i} \cap K^{*}\right)\right) & \leq \mathcal{H}^{n}\left(f\left(S_{i} \cap K^{*} \cap \partial^{*} E\right)\right)+\operatorname{Lip}(f)^{n} \omega_{*}\left(r_{i}\right) r_{i}^{n} \\
& \leq \mathcal{H}^{n}\left(f\left(S_{i} \cap \partial^{*} E\right)\right)+C \omega_{*}\left(r_{*}(\sigma)\right) r_{i}^{n} .
\end{aligned}
$$


Combining these last two estimates with (4.16), we find

$$
\begin{array}{rl}
\sum_{i} \theta_{i} \mathcal{H}^{n}\left(f\left(S_{i} \cap K^{*}\right)\right) \leq \sum_{i} & 2 \mathcal{H}^{n}\left(f\left(S_{i} \cap\left(K \backslash \partial^{*} E\right)\right)\right)+\mathcal{H}^{n}\left(f\left(S_{i} \cap \partial^{*} E\right)\right) \\
& +C \omega_{*}\left(r_{*}(\sigma)\right) \sum_{i} r_{i}^{n} \\
\leq \mathcal{F}\left(f(K), f(E) ; \bigcup_{i} f\left(S_{i}\right)\right)+C \omega_{*}\left(r_{*}(\sigma)\right),
\end{array}
$$

where $f\left(\partial^{*} E\right)=\partial^{*} f(E)$ by Lemma A.1. Combining this last estimate with 4.23 we find

$$
\begin{aligned}
& \mathcal{H}^{n}\left(\Omega \cap \partial f\left(F_{j}\right)\right) \\
& \quad \leq e^{\Lambda r_{*}(\sigma)}\left\{\mathcal{F}(f(K), f(E))+C\left\{\omega_{*}\left(r_{*}(\sigma)\right)+\tau+C(n, \tau) \sigma\right\}\right\}+\mathrm{o}(1)
\end{aligned}
$$

where o(1) $\rightarrow 0$ as first $\eta \rightarrow 0^{+}$and then $j \rightarrow \infty$; in particular, (4.18) holds.

We now conclude the proof. As explained, 4.18) implies 4.1). By a classical first variation argument (see Appendix $\mathbb{C}$ ), we deduce the existence of $\lambda \in \mathbb{R}$ such that

$$
\lambda \int_{\partial^{*} E} X \cdot v_{E} d \mathcal{H}^{n}=\int_{\partial^{*} E} \operatorname{div}^{K} X d \mathcal{H}^{n}+2 \int_{K \backslash \partial^{*} E} \operatorname{div}^{K} X d \mathcal{H}^{n}
$$

for every $X \in C_{c}^{1}\left(\mathbb{R}^{n+1} ; \mathbb{R}^{n+1}\right)$ with $X \cdot v_{\Omega}=0$ on $\partial \Omega$. Let us now consider the integer rectifiable varifold $V$ supported on $K$, with density 2 on $K \backslash \partial^{*} E$ and 1 on $\partial^{*} E$. By (4.24), we can compute the first variation of $V$ as

$$
\delta V(X)=\int \vec{H} \cdot X d\|V\| \quad \forall X \in C_{c}^{1}\left(\Omega ; \mathbb{R}^{n+1}\right)
$$

where $\vec{H}=0$ on $K \backslash \partial^{*} E$ and $\vec{H}=\lambda v_{E}$ on $\partial^{*} E$. In particular, $\vec{H} \in L^{\infty}(\|V\|)$, and by Allard's regularity theorem [47, chap. 5], we have $K=\Sigma \cup$ Reg, where $\Sigma \subset K$ is closed and has empty interior in $K$, and where for every $x \in$ Reg there exists a $C^{1, \alpha}$-function $u$ defined on $\mathbb{R}^{n}$ such that

$$
B_{r_{x} / 2}(x) \cap K=B_{r_{x} / 2}(x) \cap \operatorname{Reg}=B_{r_{x} / 2}(x) \cap \operatorname{graph}(u) .
$$

By the divergence theorem, if $x \in \operatorname{Reg} \cap \partial E$, then, by 4.25) and by $\Omega \cap \partial E \subset K$,

$$
\begin{array}{ll}
E=\operatorname{epigraph}(u) & \text { inside } B_{r_{x} / 2}(x), \\
K=\partial E=\operatorname{graph}(u) & \text { inside } B_{r_{x} / 2}(x),
\end{array}
$$

which imply Reg $\cap \partial E \subset \Omega \cap \partial^{*} E$. Conversely, if $x \in \Omega \cap \partial^{*} E$, then $\mathcal{H}^{n}\left(B_{r}(x) \cap\right.$ $\left.\left(K \backslash \partial^{*} E\right)\right)=\mathrm{o}\left(r^{n}\right)$ and $\mathcal{H}^{n}\left(B_{r}(x) \cap \partial^{*} E\right)=\omega_{n} r^{n}+\mathrm{o}\left(r^{n}\right)$ as $r \rightarrow 0^{+}$, so that Allard's regularity theorem implies $\Omega \cap \partial^{*} E \subset \operatorname{Reg} \cap \partial E$. Thus Reg $\cap \partial E=$ $\Omega \cap \partial^{*} E$, and, in particular, $\Omega \cap\left(\partial E \backslash \partial^{*} E\right) \subset \Sigma$, so that $\Omega \cap\left(\partial E \backslash \partial^{*} E\right)$ has empty interior in $K$. Moreover, by (4.26, 4.24) implies that the graph of $u$ has constant mean curvature in $B_{r_{x} / 2}(x)$, and thus that $\partial^{*} E$ is a smooth hypersurface; see, e.g., [27, sec. 8.2]. Finally, 4.24] implies that $K \backslash \partial E$ is the support of a 
multiplicity 1 stationary varifold in the open set $\Omega \backslash \partial E$, so that $K \backslash(\Sigma \cup \partial E)$ is a smooth hypersurface with zero mean curvature and $\mathcal{H}^{n}(\Sigma \backslash \partial E)=0$. The proof of Theorem 1.6 is complete.

\section{Convergence to Plateau's Problem: Proof of Theorem 1.9}

This section is devoted to showing that $\psi(\varepsilon) \rightarrow 2 \ell$ as $\varepsilon \rightarrow 0^{+}$and that a sequence $\left\{\left(K_{h}, E_{h}\right)\right\}_{h}$ of generalized minimizers for $\psi\left(\varepsilon_{h}\right)$ with $\varepsilon_{h} \rightarrow 0^{+}$as $h \rightarrow$ $\infty$ has to converge to a minimizer $S$ for Plateau's problem $\ell$ counted with multiplicity 2 in the sense of Radon measures. If one could prove the latter assertion directly, then the former would follow at once by lower semicontinuity of weakstar converging Radon measures and by the upper bound $\psi(\varepsilon) \leq 2 \ell+C \varepsilon^{n /(n+1)}$ proved in (3.2). A possible direct approach to the convergence of $\left(K_{h}, E_{h}\right)$ to a minimizer of Plateau's problem may be tried using White's compactness theorem [50]. That would require proving an $L^{1}$-bound on the first variations of the varifolds $V_{h}$ supported on $K_{h}$ with density 1 on $\Omega \cap \partial^{*} E_{h}$ and with density 2 on $K_{h} \backslash \partial^{*} E_{h}$. The validity of such bound is supported by the analysis of simple examples like Example 1.1 and Example 1.2. However, Example 1.2 also indicates that when singularities are present in the limit Plateau minimizers $S$, then an $L^{1}$-bound for the mean curvatures of the varifolds $V_{h}$ would result from a quantitative balance between the rate of divergence towards $-\infty$ of the constant mean curvatures of the reduced boundaries $\partial^{*} E_{h}$ and the rate of vanishing of the areas $\mathcal{H}^{n}\left(\Omega \cap \partial^{*} E_{h}\right)$. Validating a quantitative analysis of this kind in some generality would be of course very interesting per se as a way to describe the behavior of generalized minimizers; nonetheless, completing this analysis has so far eluded our attempts.

Coming back to the proof of Theorem 1.9, we adopt a different approach. We prove directly that $\psi(\varepsilon) \rightarrow 2 \ell$ as $\varepsilon \rightarrow 0^{+}$by exploiting the same "compactnessby-comparison" strategy adopted in the proof of Theorem 1.4. An interesting point here is that because $\left|E_{h}\right|=\varepsilon_{h} \rightarrow 0^{+}$, we do not have a limit set that we can use to uniformly adjust volumes among local competitors of the elements of the minimizing sequence, and have to use a sort of "absolute minimality at vanishing volumes" of any sequence $\left\{\left(K_{h}, E_{h}\right)\right\}_{h}$ of generalized minimizers such that $\lim _{h \rightarrow \infty} \mathcal{F}\left(K_{h}, E_{h}\right)$ is equal to $\liminf _{\varepsilon \rightarrow 0^{+}} \psi(\varepsilon)$.

\section{PROOF OF THEOREM 1.9.}

Step 1 . We start by proving that $\psi$ is lower-semicontinuous on $(0, \infty)$. Given $\varepsilon_{0}>0$, let $\varepsilon_{j} \rightarrow \varepsilon_{0}>0$ as $j \rightarrow \infty$ be such that

$$
\lim _{j \rightarrow \infty} \psi\left(\varepsilon_{j}\right)=\liminf _{\varepsilon \rightarrow \varepsilon_{0}} \psi(\varepsilon),
$$

and let $E_{j} \in \mathcal{E}$ be such that $\left|E_{j}\right|=\varepsilon_{j}$ and $\mathcal{H}^{n}\left(\Omega \cap \partial E_{j}\right) \leq \psi\left(\varepsilon_{j}\right)+1 / j$. By (3.2), $\psi\left(\varepsilon_{j}\right)$ is bounded in $j$, and thus by the compactness criteria for sets of finite perimeter and for Radon measures we have that, up to extracting subsequences, 
$\mu_{j}=\mathcal{H}^{n}\left\llcorner\left(\Omega \cap \partial E_{j}\right) \stackrel{*}{\rightarrow} \mu\right.$ as Radon measures in $\Omega$ and $E_{j} \rightarrow E$ in $L_{\mathrm{loc}}^{1}(\Omega)$, where $\mu$ is a Radon measure in $\Omega$, and where $E \subset \Omega$ is a set of finite perimeter. We now repeat the proof of Theorem 1.4 , with the only difference being that while $\left|E_{j}\right|$ was constant in that proof, we now have that $\left|E_{j}\right|=\varepsilon_{j} \rightarrow \varepsilon_{0}$ for some $\varepsilon_{0}>0$. The modifications are minimal. In Step 2 (nucleation of the sequence $E_{j}$ ), we repeat verbatim the argument, using the facts that $\left|E_{j}\right| \geq \varepsilon_{0} / 2$ and that $\mathcal{H}^{n}\left(\Omega \cap \partial E_{j}\right) \leq$ $2 \ell+C \varepsilon_{0}^{n /(n-1)}+1$ in place of $\left|E_{j}\right|=\varepsilon$ and $\mathcal{H}^{n}\left(\Omega \cap \partial E_{j}\right) \leq \psi(\varepsilon)+1$. Based on Step 2, in Step 3 we construct volume-fixing variations with uniform constant $\varepsilon_{*}$ and $C_{*}$, and then repeat the rest of the argument without modifications. As a consequence, we can show that $\mu=\theta \mathcal{H}^{n}\llcorner K$ and $(K, E) \in \mathcal{K}$ is a generalized minimizer of $\psi\left(\varepsilon_{0}\right)$, with

$$
\psi\left(\varepsilon_{0}\right)=\mu(\Omega)=\lim _{j \rightarrow \infty} \mu_{j}(\Omega) \leq \lim _{j \rightarrow \infty} \psi\left(\varepsilon_{j}\right)=\liminf _{\varepsilon \rightarrow \varepsilon_{0}} \psi(\varepsilon)
$$

as claimed. The key information here is of course that $\left|E_{j}\right| \geq \varepsilon_{0} / 2$ where $\varepsilon_{0}>0$. If $\varepsilon_{0}=0$, then the nucleation lemma is inconsequential, and the argument cannot be used.

Step 2. Thanks to (3.2), to prove $\psi(\varepsilon) \rightarrow 2 \ell$ as $\varepsilon \rightarrow 0^{+}$we just need to show that

$$
\liminf _{\varepsilon \rightarrow 0^{+}} \psi(\varepsilon) \geq 2 \ell
$$

To this end, we pick a sequence $\varepsilon_{h} \rightarrow 0^{+}$such that

$$
\liminf _{\varepsilon \rightarrow 0^{+}} \psi(\varepsilon)=\lim _{h \rightarrow \infty} \psi\left(\varepsilon_{h}\right) .
$$

Notice that, in this way, given an arbitrary sequence $\sigma_{h} \rightarrow 0^{+}$, we have

$$
\limsup _{h \rightarrow \infty}\left[\psi\left(\varepsilon_{h}\right)-\psi\left(\sigma_{h}\right)\right] \leq 0 .
$$

Let $\left\{E_{h, j}\right\}_{j}$ be a minimizing sequence in $\psi\left(\varepsilon_{h}\right)$. By Theorem 1.4 there exists a generalized minimizer $\left(K_{h}, E_{h}\right)$ in $\psi\left(\varepsilon_{h}\right)$ such that, up to extracting subsequences,

$$
\begin{array}{cc}
E_{h, j} \rightarrow E_{h} & \text { in } L^{1}(\Omega) \text { as } j \rightarrow \infty, \\
\mu_{h, j} z=\mathcal{H}^{n}\left\llcorner\left(\Omega \cap \partial E_{h, j}\right) \stackrel{*}{\rightarrow} \mu_{h} \quad \text { as Radon measures in } \Omega \text { as } j \rightarrow \infty,\right. \\
\left|E_{h, j}\right|=\varepsilon_{h} \quad \text { and } \quad \mathcal{H}^{n}\left(\Omega \cap \partial E_{h, j}\right) \leq \psi\left(\varepsilon_{h}\right)+\frac{1}{j} \quad \forall j \in \mathbb{N}
\end{array}
$$

where, by (3.2) and up to extracting a further subsequence,

$$
\mu_{h}=2 \mathcal{H}^{n}\left\llcorner\left(K_{h} \backslash \partial^{*} E_{h}\right)+\mathcal{H}^{n}\left\llcorner\left(\Omega \cap \partial^{*} E_{h}\right) \stackrel{*}{\rightarrow} \mu\right.\right.
$$
as Radon measures in $\Omega$. 
Given $x \in \Omega \cap \operatorname{spt} \mu$, we set $d(x)=\operatorname{dist}(x, \partial \Omega)$, and let

$$
\begin{aligned}
H_{x, r} & =\left\{h \in \mathbb{N}:\left|E_{h} \backslash B_{r}(x)\right|>0\right\}, \\
I_{x} & =\left\{r \in(0, d(x)): H_{x, r} \text { is infinite }\right\} .
\end{aligned}
$$

We now look at local variations $F_{h, j}$ of $E_{h, j}$ such that $\left|F_{h, j}\right|$ has a positive limit volume $\sigma_{h}$ as $j \rightarrow \infty$, which in turn satisfies $\sigma_{h} \rightarrow 0^{+}$as $h \rightarrow \infty$. The idea is that, by (5.3), we will be able to use such variations to gather information on $\mu$.

Claim: For every $r \in I_{x}$, if $\left\{F_{h, j}\right\}_{h \in H_{x, r}, j \in \mathbb{N}} \subset \mathcal{E}$ is such that $\Omega \cap \partial F_{h, j}$ is $\mathcal{C}$ spanning $W$ and $F_{h, j} \Delta E_{h, j} \subset \operatorname{cl}\left(B_{r}(x)\right)$ for every $h \in H_{x, r}$ and every $j \in \mathbb{N}$, and if

$$
\exists \sigma_{h}=\lim _{j \rightarrow \infty}\left|F_{h, j}\right|>0 \text { and } \lim _{h \in H_{x, r} h \rightarrow \infty} \sigma_{h}=0,
$$

then

$$
\mu\left(B_{r}(x)\right) \leq \liminf _{h \in H_{x, r} h \rightarrow \infty} \liminf _{j \rightarrow \infty} \mathcal{H}^{n}\left(\operatorname{cl}\left(B_{r}(x)\right) \cap \partial F_{h, j}\right) .
$$

To prove this claim, we first notice that, for every $h \in H_{x, r}$,

$$
\sigma_{h}=\lim _{j \rightarrow \infty}\left|F_{h, j}\right| \geq\left|E_{h} \backslash B_{r}(x)\right|>0 .
$$

In particular, for $j$ large enough, $\left|F_{h, j}\right|>0, \psi\left(\left|F_{h, j}\right|\right)$ is well-defined, and $F_{h, j}$ is a competitor for $\psi\left(\left|F_{h, j}\right|\right)$, so that

$$
\begin{aligned}
\psi\left(\left|F_{h, j}\right|\right) & \leq \mathcal{H}^{n}\left(\Omega \cap \partial F_{h, j}\right) \\
& =\mathcal{H}^{n}\left(\operatorname{cl}\left(B_{r}(x)\right) \cap \partial F_{h, j}\right)+\mathcal{H}^{n}\left(\partial E_{h, j} \cap \Omega \backslash \operatorname{cl}\left(B_{r}(x)\right)\right) \\
& \leq \mathcal{H}^{n}\left(\operatorname{cl}\left(B_{r}(x)\right) \cap \partial F_{h, j}\right)+\psi\left(\varepsilon_{h}\right)+\frac{1}{j}-\mathcal{H}^{n}\left(\partial E_{h, j} \cap B_{r}(x)\right),
\end{aligned}
$$

which can be recombined into

$$
\mu_{h, j}\left(B_{r}(x)\right) \leq \mathcal{H}^{n}\left(\operatorname{cl}\left(B_{r}(x)\right) \cap \partial F_{h, j}\right)+\psi\left(\varepsilon_{h}\right)-\psi\left(\left|F_{h, j}\right|\right)+\frac{1}{j} .
$$

Letting $j \rightarrow \infty$, by $\mu_{h, j} \stackrel{*}{\rightarrow} \mu_{h},\left|F_{h, j}\right| \rightarrow \sigma_{h}>0$, and the lower semicontinuity of $\psi$ on $(0, \infty)$, we find that

$$
\mu_{h}\left(B_{r}(x)\right) \leq \liminf _{j \rightarrow \infty} \mathcal{H}^{n}\left(\operatorname{cl}\left(B_{r}(x)\right) \cap \partial F_{h, j}\right)+\psi\left(\varepsilon_{h}\right)-\psi\left(\sigma_{h}\right) .
$$

Since $\sigma_{h} \rightarrow 0^{+}$as $h \rightarrow \infty$ with $h \in H_{x, r}$, by $\mu_{h} \stackrel{*}{\rightarrow} \mu$ and (5.3) we deduce (5.7), and thus prove the claim.

Step 3. We now fix $x \in \operatorname{spt} \mu$, set $f(r)=\mu\left(B_{r}(x)\right)$, and prove that, for a.e. $r \in I_{x}$

$$
\left\{\begin{array}{l}
\text { either } f^{\prime}(r) \geq c(n) r^{n-1} \\
\text { or }\left(f^{1 / n}\right)^{\prime}(r) \geq c(n)
\end{array}\right.
$$




$$
f(r) \leq \frac{r}{n} f^{\prime}(r)
$$

By using the coarea formula together with $\left|E_{h}\right| \rightarrow 0$ as $h \rightarrow \infty$ and $E_{h, j} \rightarrow E_{h}$ as $j \rightarrow \infty$, we find that for a.e. $r<d(x)$,

$$
\begin{gathered}
\partial E_{h, j} \cap \partial B_{r}(x) \text { is } \mathcal{H}^{n-1} \text {-rectifiable, } \\
\lim _{j \rightarrow \infty} \mathcal{H}^{n}\left(E_{h, j} \cap \partial B_{r}(x)\right)=\mathcal{H}^{n}\left(E_{h} \cap \partial B_{r}(x)\right), \\
\lim _{h \rightarrow \infty} \lim _{j \rightarrow \infty} \mathcal{H}^{n}\left(E_{h, j} \cap \partial B_{r}(x)\right)=0,
\end{gathered}
$$

for every $h, j \in \mathbb{N}$. Moreover, if we set

$$
f_{h, j}(r)=\mu_{h, j}\left(B_{r}(x)\right), \quad f_{h}(r)=\mu_{h}\left(B_{r}(x)\right) .
$$

then, again by the coarea formula and by Fatou's lemma, for a.e. $r<d(x)$ we find

$$
\begin{gathered}
\mathcal{H}^{n-1}\left(\partial E_{h, j} \cap \partial B_{r}(x)\right) \leq f_{h, j}^{\prime}(r), \\
g_{h}(r)=\liminf _{j \rightarrow \infty} f_{h, j}^{\prime}(r) \leq f_{h}^{\prime}(r), \\
g(r)=\liminf _{h \in H_{x, r}, h \rightarrow \infty} f_{h}^{\prime}(r) \leq f^{\prime}(r),
\end{gathered}
$$

for every $h, j \in \mathbb{N}$. We first prove (5.9). Let $r \in I_{x}$ be such that (5.11), (5.12), (5.13), and (5.14) hold, and let $A_{h, j}$ denote an $\mathcal{H}^{n}$-maximal connected component of $\partial B_{r}(x) \backslash \partial E_{h, j}$. If $A_{h, j} \subset E_{h, j}$, then, by spherical isoperimetry, by (5.14), and since the relative boundary to $A_{h, j}$ in $\partial B_{r}(x)$ is contained in $\partial B_{r}(x) \cap \partial E_{h, j}$, we find

$$
f_{h, j}^{\prime}(r) \geq c(n) \mathcal{H}^{n}\left(\partial B_{r}(x) \backslash A_{h, j}\right)^{(n-1) / n},
$$

where the lower bound converges to $c(n) r^{n-1}$ if we let first $j \rightarrow \infty$ and then $h \rightarrow \infty$ thanks to 5.13; hence, if $A_{h, j} \subset E_{h, j}$, the first alternative in 5.9) holds. We now assume that $A_{h, j} \cap E_{h, j}=\varnothing$, and consider the corresponding cup competitor $F_{h, j}$ as defined in Lemma 2.5 starting from $E_{h, j}, A_{h, j}$. More precisely, if $\left\{\eta_{k}^{h, j}\right\}_{k=1}^{\infty}$ denotes the corresponding sequence as in (2.49), we choose $k(h, j)$ so that, setting

$$
\begin{aligned}
& Y_{h, j}=\partial B_{r}(x) \backslash \operatorname{cl}\left(\left(E_{h, j} \cap \partial B_{r}(x)\right) \cup A_{h, j}\right), \\
& S_{h, j}=\partial E_{h, j} \cap \operatorname{cl}\left(A_{h, j}\right) \backslash\left(\operatorname{cl}\left(\left(E_{h, j} \cap \partial B_{r}(x)\right) \cup Y_{h, j}\right)\right),
\end{aligned}
$$

we have that $\eta_{j}=\eta_{k(h, j)}^{h, j}$ satisfies $\eta_{j} \leq \frac{r}{2 j}$, with

$$
\begin{aligned}
& \mathcal{H}^{n}\left(\partial B_{r}(x) \cap\left\{\mathrm{d}_{S_{h, j}} \leq \eta_{j}\right\}\right) \leq \frac{1}{j}, \\
& \eta_{j} \mathcal{H}^{n-1}\left(\partial B_{r}(x) \cap\left\{\mathrm{d}_{S_{h, j}}=\eta_{j}\right\}\right) \leq \frac{1}{j} .
\end{aligned}
$$


Then, with the usual notation

$$
U_{h, j}=\partial B_{r}(x) \cap\left\{\mathrm{d}_{S_{h, j}}<\eta_{j}\right\}, \quad Z_{h, j}=Y_{h, j} \cup\left(U_{h, j} \backslash \operatorname{cl}\left(E_{h, j} \cap \partial B_{r}(x)\right)\right),
$$

we define

$$
F_{h, j}=\left(E_{h, j} \backslash \operatorname{cl}\left(B_{r}(x)\right)\right) \cup N_{\eta_{j}}\left(Z_{h, j}\right) .
$$

By Lemma 2.5. $F_{h, j} \in \mathcal{E}, \Omega \cap \partial F_{h, j}$ is $\mathcal{C}$-spanning $W$ and $E_{h, j} \Delta F_{h, j} \subset \operatorname{cl}\left(B_{r}(x)\right)$. Since $\eta_{j} \rightarrow 0$ as $j \rightarrow \infty$, we find

$$
\sigma_{h}=\lim _{j \rightarrow \infty}\left|F_{h, j}\right|=\lim _{j \rightarrow \infty}\left|E_{h, j} \backslash B_{r}(x)\right|=\left|E_{h} \backslash B_{r}(x)\right|,
$$

so that $\sigma_{h}>0$ if $h \in H_{x, r}$, and $\sigma_{h} \rightarrow 0^{+}$if we let $h \rightarrow \infty$. Thus $F_{h, j}$ satisfies (5.6), and we can apply (5.7) to $F_{h, j}$. To estimate the upper bound in (5.7), we look back at 2.40), 2.43), 2.44), and 2.47), and find that

$$
\begin{aligned}
\mathcal{H}^{n}\left(\operatorname{cl}\left(B_{r}(x)\right) \cap \partial F_{h, j}\right) & \\
\leq & \left(2+C(n) \eta_{j}\right) \mathcal{H}^{n}\left(\partial B_{r}(x) \backslash A_{h, j}\right) \\
& +\left(2+C(n) \eta_{j}\right) \mathcal{H}^{n}\left(\partial B_{r}(x) \cap\left\{\mathrm{d}_{S_{h, j}} \leq \eta_{j}\right\}\right) \\
& +C\left(n, \eta_{j}\left(\mathcal{H}^{n-1}\left(\partial B_{r}(x) \cap \partial E_{h, j}\right)\right.\right. \\
& \left.+\mathcal{H}^{n-1}\left(\partial B_{r}(x) \cap\left\{\mathrm{d}_{S_{h, j}}=\eta_{j}\right\}\right)\right) .
\end{aligned}
$$

By (5.7), 5.15), 5.16, and 5.17) we deduce that

$$
\begin{aligned}
f(r)=\mu\left(B_{r}(x)\right) & \leq \liminf _{h \in H_{x, r}, h \rightarrow \infty} \liminf _{j \rightarrow \infty} \mathcal{H}^{n}\left(\operatorname{cl}\left(B_{r}(x)\right) \cap \partial F_{h, j}\right) \\
& \leq \liminf _{h \in H_{x, r}, h \rightarrow \infty} \liminf _{j \rightarrow \infty} 2 \mathcal{H}^{n}\left(\partial B_{r}(x) \backslash A_{h, j}\right) \\
& \leq C(n) \liminf _{h \in H_{x, r}, h \rightarrow \infty} \liminf _{j \rightarrow \infty} f_{h, j}^{\prime}(r)^{n /(n-1)} \\
& \leq C(n) f^{\prime}(r)^{n /(n-1)} .
\end{aligned}
$$

We have thus proved that the second alternative in $(5.9)$ holds, as claimed. We now prove 5.10): let us now denote by $F_{h, j}$ the set defined by Lemma 2.9 as approximation of the cone competitor corresponding to $E_{h, j}$ in $B_{r}(x)$ with $\eta=\eta_{j}=$ $r / 2 j$. We have that $F_{h, j} \in \mathcal{E}$ and that $\Omega \cap \partial F_{h, j}$ is $\mathcal{C}$-spanning $W$; furthermore, by (2.72) and 5.12) we find

$$
\sigma_{h}=\lim _{j \rightarrow \infty}\left|F_{h, j}\right| \geq\left|E_{h} \backslash B_{r}(x)\right|+\frac{r}{n+1} \mathcal{H}^{n}\left(E_{h} \cap \partial B_{r}(x)\right)
$$

(in particular, $\sigma_{h}>0$ if $h \in H_{x, r}$ ) and, by (5.13), $\sigma_{h} \rightarrow 0^{+}$as $h \rightarrow \infty$. Thus (5.6) holds, and we can deduce from (5.7) and (2.71) that

$$
f(r)=\mu\left(B_{r}(x)\right) \leq \liminf _{h \in H_{x, r}, h \rightarrow \infty} \liminf _{j \rightarrow \infty} \frac{r}{n} \mathcal{H}^{n-1}\left(\partial E_{h, j} \cap \partial B_{r}(x)\right) \leq \frac{r}{n} f^{\prime}(r),
$$

that is 5.10 . 
Step 4. We now define a function $g: \Omega \rightarrow(0, \infty) \cup\{-\infty\}$ by letting

$$
\begin{aligned}
g(x) & =\sup \left\{s>0:(0, s) \subset I_{x}\right\} \\
& =\sup \left\{t>0: \text { if } s<t, \text { then }\left|E_{h} \backslash B_{s}(x)\right|>0 \text { for infinitely many } h\right\} .
\end{aligned}
$$

We notice that

$g$ is lower semicontinuous on $\Omega$,

$\{g=-\infty\}$ contains at most one point.

(Notice that $\{g=-\infty\}$ may indeed contain one point: this is the case of the singular point of a triple junction; see Figure 1.3-(b)). To prove (5.19): if $g(x) \neq$ $-\infty$, then $g(x)>0$, and for every $s \in(0, g(x)),\left|E_{h} \backslash B_{s}(x)\right|>0$ for infinitely many $h$. Thus, if $\eta \in(0, g(x))$ and $m_{\eta}$ is such that $\left|x-x_{m}\right|<\eta$ for every $m \geq m_{\eta}$, then, for every $m \geq m_{\eta}$ and $s \in(0, g(x)-\eta)$,

$$
\left|E_{h} \backslash B_{S}\left(x_{m}\right)\right| \geq\left|E_{h} \backslash B_{s+\eta}(x)\right|>0, \quad \text { for infinitely many } h,
$$

that is $g(x)-\eta \leq g\left(x_{m}\right)$ for every $m \geq m_{\eta}$; this proves 5.19). Next, if $g\left(x_{1}\right)=$ $g\left(x_{2}\right)=-\infty$, then for every $s>0$ there exists $h(s)$ such that

$$
\left|E_{h} \backslash B_{s}\left(x_{1}\right)\right|=\left|E_{h} \backslash B_{S}\left(x_{2}\right)\right|=0 \quad \forall h \geq h(s) .
$$

If $x_{1} \neq x_{2}$ we can take $s=\left|x_{1}-x_{2}\right| / 2$ and deduce $\left|E_{h}\right|=0$; thus (5.20) holds.

Let us now consider the open set $\{g>s\} \subset \Omega, s>0$, and set

$$
Z(s)=\operatorname{spt} \mu \cap\{g>s\}, \quad Z=\operatorname{spt} \mu \cap\{g>0\} .
$$

We claim that if $x \in Z(s)$, then

$$
f(r) \geq c_{0}(n) r^{n} \quad \forall r \in(0, s), r^{-n} f(r) \text { increasing over } r \in(0, s) .
$$

The second assertion is immediate from (5.10). To prove the first one, set

$$
L_{1}=\left\{r \in(0, s): f^{\prime}(r) \geq c(n) r^{n-1}\right\}, \quad L_{2}=(0, s) \backslash L_{1},
$$

with $c(n)$ as in 5.9. If $x \in Z(s)$ is such that $\mathcal{H}^{1}\left(L_{1}\right) \geq s / 2$, then for every $r \in(0, s)$

$$
\begin{aligned}
f(r) \geq \int_{L_{1} \cap(0, r)} f^{\prime} & \geq c(n) \int_{L_{1} \cap(0, r)} t^{n-1} d t \\
& \geq c(n) \int_{0}^{\min \{r, s / 2\}}, t^{n-1} d t \geq \frac{c(n)}{n 2^{n}} r^{n} ;
\end{aligned}
$$

if instead $\mathcal{H}^{1}\left(L_{2}\right) \geq s / 2$, then for every $r \in(0, s)$,

$$
\begin{aligned}
f(r)^{1 / n} \geq \int_{L_{2} \cap(0, r)}\left(f^{1 / n}\right)^{\prime} & \geq c(n) \mathcal{H}^{1}\left(L_{2} \cap(0, r)\right) \\
& \geq c(n) \min \left\{r, \frac{s}{2}\right\} \geq \frac{c(n)}{2} r,
\end{aligned}
$$

where we have used the fact that, by (5.9), we have $\left(f^{1 / n}\right)^{\prime} \geq c(n)$ on $L_{2}$. Thanks to (5.21) we are in the position of using [45, theorem 6.9] and Preiss' theorem 
(as done in Step 4 of the proof of Theorem 1.4) on each $Z(s)$, to find that $Z$ is $\mathcal{H}^{n}$-rectifiable with

$$
\mu\left\llcorner Z=\theta \mathcal{H}^{n}\llcorner Z,\right.
$$

where the density

$$
\theta(x)=\lim _{r \rightarrow 0^{+}} \frac{\mu\left(B_{r}(x)\right)}{\omega_{n} r^{n}} \quad \text { exists in }\left[c_{0}(n), \infty\right) \text { for every } x \in Z .
$$

Moreover, by (5.20),

$$
\mathcal{H}^{0}(\operatorname{spt} \mu \backslash Z) \leq 1 \text {. }
$$

By combining (5.22) and (5.23) we find that $K=\operatorname{spt} \mu$ is $\mathcal{H}^{n}$-rectifiable and such that $\mu=\theta \mathcal{H}^{n}\left\llcorner K\right.$. Since $K_{h}=\operatorname{spt} \mu_{h}$ is $\mathcal{C}$-spanning $W$ and $\mu_{h} \stackrel{*}{\rightarrow} \mu$, by Lemma 2.1 we find that $K$ is $\mathcal{C}$-spanning $W$, and thus admissible in $\ell$, so that

$$
\begin{aligned}
\liminf _{\varepsilon \rightarrow 0^{+}} \psi(\varepsilon)=\lim _{h \rightarrow \infty} \mu_{h}(\Omega) & \geq \mu(\Omega) \\
& =\int_{K} \theta d \mathcal{H}^{n} \geq \min _{K} \theta \mathcal{H}^{n}(K) \geq \ell \min _{K} \theta .
\end{aligned}
$$

Thus, to complete the proof of 5.1) we just need to show that

$$
\theta \geq 2 \mathcal{H}^{n} \text {-a.e. on } K \text {. }
$$

Since $\mu_{h, j}=\mathcal{H}^{n}\left\llcorner\left(\Omega \cap \partial E_{h, j}\right) \stackrel{*}{\rightarrow} \mu_{h}\right.$ as $j \rightarrow \infty$, with $\mu_{h} \stackrel{*}{\rightarrow} \theta \mathcal{H}^{n}\llcorner K$ as $h \rightarrow \infty$, we can extract a diagonal subsequence $j=j(h)$ so that, denoting $E_{h}^{*}=$ $E_{h, j(h)},\left\{E_{h}^{*}\right\}_{h} \subset \mathcal{E}, \Omega \cap \partial E_{h}^{*} \mathcal{C}$-spanning $W$, and

$$
\mu_{h}^{*}=\mathcal{H}^{n}\left\llcorner\left(\Omega \cap \partial E_{h}^{*}\right) \stackrel{*}{\rightarrow} \theta \mathcal{H}^{n}\llcorner K \quad \text { as } h \rightarrow \infty .\right.
$$

Moreover, $\mu\left(B_{r}(x)\right) \geq c(n) r^{n}$ for every $r \in(0, s)$ if $x \in K \cap\{g>s\}$ and, thanks to 5.17,

$$
\liminf _{h \rightarrow \infty} \mathcal{H}^{n}\left(B_{r}(x) \cap \partial E_{h}^{*}\right) \leq C(n) \liminf _{h \rightarrow \infty} \mathcal{H}^{n}\left(\partial B_{r}(x) \backslash A_{r, h}^{0}\right)
$$

where $A_{r, h}^{0}$ denotes an $\mathcal{H}^{n}$-maximal connected component of $\partial B_{r}(x) \backslash \partial E_{h}^{*}$, this time for every $x \in K$ and $B_{r}(x) \Subset \Omega$. We can thus apply Lemma 2.11 with the open set $\Omega^{\prime}=\{g>s\}$ to deduce that

$$
\theta \geq 2 \mathcal{H}^{n} \text {-a.e. on }\{g>s\} \cap K \backslash \partial^{*} E^{*}
$$

where $E^{*}=\varnothing$ is the $L^{1}$-limit of the sets $E_{h}^{*}$. Since $\partial^{*} E^{*}=\varnothing$, taking the union over $s>0$ and recalling (5.23), we conclude that (5.25) holds.

Step 5. Now that $\psi(\varepsilon) \rightarrow 2 \ell$ as $\varepsilon \rightarrow 0^{+}$has been proved, let $\left(K_{h}, E_{h}\right)$ be a sequence of generalized minimizers of $\psi\left(\varepsilon_{h}\right)$ for an arbitrary sequence $\varepsilon_{h} \rightarrow 0^{+}$. Since the limit of $\psi(\varepsilon)$ as $\varepsilon \rightarrow 0^{+}$exists, $\varepsilon_{h}$ automatically satisfies 5.2 , and the arguments of Steps 2 to 4 can be repeated verbatim. Correspondingly, up to extracting subsequences, (5.4) holds with $\mu=\theta \mathcal{H}^{n}\left\llcorner K, \theta \geq 2 \mathcal{H}^{n}\right.$-a.e. on $K$, and $K$ a relatively compact subset of $\Omega, \mathcal{H}^{n}$-rectifiable, and $\mathcal{C}$-spanning $W$. By 
plugging $\psi(\varepsilon) \rightarrow 2 \ell$ as $\varepsilon \rightarrow 0^{+}$in (5.24), we find that $\theta=2 \mathcal{H}^{n}$-a.e. on $K$, $2 \mathcal{H}^{n}(K)=2 \ell$, so that $K$ is a minimizer of $\ell$, and thus, looking back at (5.4), we conclude that $(1.18)$ holds.

\section{Appendix A A Technical Fact on Sets of Finite Perimeter}

LEMMA A.1. If $\Omega$ is an open set in $\mathbb{R}^{n+1}, E$ is a set of finite perimeter in $\Omega$, and $f: \Omega \rightarrow \Omega$ is a diffeomorphism, then $f(E)$ is a set of finite perimeter in $\Omega$ with $\partial^{*} f(E)=f\left(\partial^{*} E\right)$ and

$$
v_{f(E)}(f(x))=\frac{(\nabla g(f(x)))^{\top} v_{E}(x)}{\left|(\nabla g(f(x)))^{\top} v_{E}(x)\right|} \quad \forall x \in \partial^{*} E
$$

where $g=f^{-1}$.

PROOF. In [42, prop. 17.1, remark 17.2] it is shown that $f(E)$ is a set of finite perimeter with

$$
\mu_{f(E)}=f_{\#}\left(J f(\nabla g(f))^{\top} \mu_{E}\right),
$$

and that mapping by $f$ preserves essential boundaries (thus just the $\mathcal{H}^{n}$-equivalence of $\partial^{*} f(E)$ and $f\left(\partial^{*} E\right)$ is deduced there). In order to prove $\partial^{*} f(E)=f\left(\partial^{*} E\right)$, we pick a ball $B_{r}(f(x)) \Subset \Omega$, and look at

$$
\begin{aligned}
\frac{\mu_{f(E)}\left(B_{r}(f(x))\right)}{\left|\mu_{f(E)}\right|\left(B_{r}(f(x))\right)} & =\frac{\int_{g\left(B_{r}(f(x))\right) \cap \partial^{*} E} J f \nabla g(f)^{\top} v_{E} d \mathcal{H}^{n}}{\int_{g\left(B_{r}(f(x))\right) \cap \partial^{*} E} J f\left|\nabla g(f)^{\top} v_{E}\right| d \mathcal{H}^{n}} \\
& =\frac{\int_{\left(\partial^{*} E-x\right) / r} u_{r}(z) v_{E}(x+r z) d \mathcal{H}_{z}^{n}}{\int_{\left(\partial^{*} E-x\right) / r}\left|u_{r}(z) v_{E}(x+r z)\right| d \mathcal{H}_{z}^{n}},
\end{aligned}
$$

where we have set

$$
F_{r}=\frac{g\left(B_{r}(f(x))\right)-x}{r}, \quad u_{r}(z)=1_{F_{r}}(z) J f(x+r z) \nabla g(f(x+r z))^{\top} .
$$

If we set $F=L\left(B_{1}(0)\right)$ for the linear map $L=\nabla g(f(x))$, then for every $\varepsilon>0$ we have

$$
L\left(B_{1-\varepsilon}(0)\right) \subset F_{r} \subset L\left(B_{1+\varepsilon}(0)\right) \text { provided } r \text { is small enough, }
$$

and thus, as $r \rightarrow 0^{+}$,

$$
1_{F_{r}} \rightarrow 1_{F} \quad \text { uniformly on } \mathbb{R}^{n+1} \backslash X_{\varepsilon}
$$

where we have set

$$
X_{\varepsilon}=L\left(B_{1+\varepsilon}(0) \backslash B_{1-\varepsilon}(0)\right) .
$$

Since $F_{r}, F \subset B_{\text {Lip } g}(0)$, and since for every $R>0$

$$
J f(x+r z) \nabla g(f(x+r z))^{\top} \rightarrow J f(x) \nabla g(f(x))^{\top} \quad \text { uniformly on }|z| \leq R
$$

as $r \rightarrow 0^{+}$, we conclude that

(A.3) $\quad u_{r}(z) \rightarrow u(z):=1_{F}(z) J f(x) \nabla g(f(x))^{\top} \quad$ uniformly on $\mathbb{R}^{n+1} \backslash X_{\varepsilon}$. 
We now decompose the integrals over $\left(\partial^{*} E-x\right) / r$ appearing in (A.2) through $X_{\varepsilon}$. By A.3,

$$
\begin{aligned}
& \mid \int_{\left[\left(\partial^{*} E-x\right) / r\right] \backslash X_{\varepsilon}} u_{r}(z) v_{E}(x+r z) d \mathcal{H}_{z}^{n} \\
& -\int_{\left[\left(\partial^{*} E-x\right) / r\right] \backslash X_{\varepsilon}} u(z) v_{E}(x+r z) d \mathcal{H}_{z}^{n} \mid \\
& \quad \leq \omega(r) \mathcal{H}^{n}\left(B_{\operatorname{Lip} g}(0) \cap\left[\left(\partial^{*} E-x\right) / r\right] \backslash X_{\mathcal{E}}\right) \\
& \quad \leq \omega(r) P\left(E ; B_{r \operatorname{Lip} g}(x)\right) \rightarrow 0
\end{aligned}
$$

as $r \rightarrow 0^{+}$, while $x \in \partial^{*} E$ gives

$$
\lim _{r \rightarrow 0^{+}} \int_{\left[\left(\partial^{*} E-x\right) / r\right] \backslash X_{\varepsilon}} u(z) v_{E}(x+r z) d \mathcal{H}_{z}^{n}=\int_{T_{x}\left(\partial^{*} E\right) \backslash X_{\varepsilon}} u(z) v_{E}(x) d \mathcal{H}_{z}^{n} .
$$

At the same time, since $\left|u_{r}\right| \leq C$ for a constant $C$ independent of $r$, we have

$$
\begin{aligned}
& \left|\int_{X_{\varepsilon} \cap\left[\left(\partial^{*} E-x\right) / r\right]} u_{r}(z) v_{E}(x+r z) d \mathcal{H}_{z}^{n}\right| \\
& \quad \leq C \mathcal{H}^{n}\left(X_{\varepsilon} \cap \frac{\partial^{*} E-x}{r}\right) \rightarrow C \mathcal{H}^{n}\left(X_{\varepsilon} \cap T_{x}\left(\partial^{*} E\right)\right)
\end{aligned}
$$

as $r \rightarrow 0^{+}$. Combining the above estimates with $|u| \leq C$, we finally find

$$
\begin{aligned}
& \limsup _{r \rightarrow 0^{+}}\left|\int_{\left(\partial^{*} E-x\right) / r} u_{r}(z) v_{E}(x+r z) d \mathcal{H}_{z}^{n}-\int_{T_{x}\left(\partial^{*} E\right)} u(z) v_{E}(x) d \mathcal{H}_{z}^{n}\right| \\
& \quad \leq C \mathcal{H}^{n}\left(X_{\varepsilon} \cap T_{x}\left(\partial^{*} E\right)\right) \quad \forall \varepsilon>0 .
\end{aligned}
$$

Letting $\varepsilon \rightarrow 0^{+}$, we find $\mathcal{H}^{n}\left(X_{\varepsilon} \cap T_{x}\left(\partial^{*} E\right)\right) \rightarrow \mathcal{H}^{n}\left(X \cap T_{x}\left(\partial^{*} E\right)\right)$ where

$$
X=L\left(\partial B_{1}(0)\right) \text {. }
$$

Since $L$ is invertible, $L\left(\partial B_{1}(0)\right)$ intersects transversally any plane through the origin, and in particular $T_{x}\left(\partial^{*} E\right)$. Therefore $\mathcal{H}^{n}\left(X \cap T_{x}\left(\partial^{*} E\right)\right)=0$, and we have proved

$$
\begin{aligned}
\lim _{r \rightarrow 0^{+}} \int_{\left(\partial^{*} E-x\right) / r} u_{r}(z) v_{E}(x+r z) d \mathcal{H}_{z}^{n} & =\int_{T_{x}\left(\partial^{*} E\right)} u(z) v_{E}(x) d \mathcal{H}_{z}^{n} \\
& =J f(x) L^{\top} v_{E}(x) \mathcal{H}^{n}\left(F \cap T_{x}\left(\partial^{*} E\right)\right) .
\end{aligned}
$$

An analogous argument shows

$$
\begin{aligned}
& \lim _{r \rightarrow 0^{+}} \int_{\left(\partial^{*} E-x\right) / r}\left|u_{r}(z) v_{E}(x+r z)\right| d \mathcal{H}_{z}^{n} \\
& \quad=J f(x)\left|L^{\top} v_{E}(x)\right| \mathcal{H}^{n}\left(F \cap T_{x}\left(\partial^{*} E\right)\right),
\end{aligned}
$$


and finally we conclude that if $x \in \partial^{*} E$, then

$$
\lim _{r \rightarrow 0^{+}} \frac{\mu_{f(E)}\left(B_{r}(f(x))\right)}{\left|\mu_{f(E)}\right|\left(B_{r}(f(x))\right)}=\frac{L^{\top} v_{E}(x)}{\left|L^{\top} v_{E}(x)\right|} \in \mathbb{S}^{n} .
$$

In particular, $f(x) \in \partial^{*} f(E)$ and (A.1) holds.

\section{Appendix B Boundary Density Estimates for the Harrison-Pugh Minimizers}

In this appendix we prove that when $\partial W$ is smooth and $\ell<\infty$, then every minimizer $S$ of $\ell$ satisfies uniform lower density estimates up to the boundary of $\Omega$.

THEOREM B.1. If $\ell<\infty, \partial W$ is smooth, and $S$ is a minimizer of $\ell$, then

$$
\mathcal{H}^{n}\left(B_{r}(x) \cap S\right) \geq c(n) r^{n} \quad \forall x \in \operatorname{cl}(S) r \in\left(0, r_{0}\right)
$$

for a value of $r_{0}$ depending on $W$.

Proof. By Lemma 2.4, and since $S$ minimizes $\mathcal{H}^{n}$ with respect to every relatively closed subset of $\Omega$ that is $\mathcal{C}$-spanning $W$ (recall $(1.8)$ ), we have

$$
\mathcal{H}^{n}(S) \leq \mathcal{H}^{n}(f(S))
$$

whenever $f: \operatorname{cl}(\Omega) \rightarrow \operatorname{cl}(\Omega)$ is a homeomorphism with $f(\partial \Omega)=\partial \Omega,\{f \neq$ id $\} \Subset B_{r_{0}}(x)$ for $x \in \partial \Omega$, and $f\left(B_{r_{0}}(x) \cap \mathrm{cl}(\Omega)\right)=B_{r_{0}}(x) \cap \mathrm{cl}(\Omega)$ for $r_{0}$ depending on $W$. We immediately deduce from (B.2), that

$$
\int_{S} \operatorname{div}^{S} X d \mathcal{H}^{n}=0
$$

for every $X \in C_{c}^{1}\left(B_{r_{0}}(x) ; \mathbb{R}^{n+1}\right)$ with $X \cdot v_{\Omega}=0$ on $\partial \Omega$. Since $S$ is an Almgren minimizer in $\Omega$, (B.3) also holds for every $X \in C_{c}^{1}\left(\Omega ; \mathbb{R}^{n+1}\right)$. Finally, we deduce the validity of $(\mathrm{B} .3)$ for every $X \in C_{c}^{1}\left(\mathbb{R}^{n+1} ; \mathbb{R}^{n+1}\right)$ with $X \cdot v_{\Omega}=0$ on $\partial \Omega$ by a standard covering argument.

The validity of $(\mathrm{B} .3)$ for every $X \in C_{c}^{1}\left(\mathbb{R}^{n+1} ; \mathbb{R}^{n+1}\right)$ with $X \cdot v_{\Omega}=0$ on $\partial \Omega$ is a distributional formulation of Young's law, which has been extensively studied in the classical work of Grüter and Jost [30], and has been recently extended to arbitrary contact angles by Kagaya and Tonegawa [36]. The main consequence of (B.3) we shall need here is an adapted monotonicity formula that takes care of the local geometry of $\partial W$. We now introduce this tool and then complete the proof.

Let $r_{0}$ be sufficiently small so that $I_{r_{0}}(\partial W)$ admits a well-defined nearest-point projection map $\Pi: I_{r_{0}}(\partial W) \rightarrow \partial W$ of class $C^{1}$. By [36, theorem 3.2], there exists a constant $C=C\left(n, r_{0}\right)$ such that for any $x \in I_{r_{0} / 6}(\partial W) \cap \operatorname{cl}(\Omega)$ the map

$$
r \in\left(0, r_{0} / 6\right) \mapsto \frac{\mathcal{H}^{n}\left(S \cap B_{r}(x)\right)+\mathcal{H}^{n}\left(S \cap \widetilde{B}_{r}(x)\right)}{\omega_{n} r^{n}} e^{C r}
$$


is increasing, where

$$
\widetilde{B}_{r}(x):=\left\{y \in \mathbb{R}^{n+1}: \tilde{y} \in B_{r}(x)\right\}, \quad \tilde{y}:=\Pi(y)+(\Pi(y)-y),
$$

denotes a sort of nonlinear reflection of $B_{r}(x)$ across $\partial W$. In particular, the limit

$$
\sigma(x)=\lim _{r \rightarrow 0^{+}} \frac{\mathcal{H}^{n}\left(S \cap B_{r}(x)\right)+\mathcal{H}^{n}\left(S \cap \widetilde{B}_{r}(x)\right)}{\omega_{n} r^{n}}
$$

exists for every $x \in I_{r_{0} / 6}(\partial W) \cap \operatorname{cl}(\Omega)$, and the map $x \mapsto \sigma(x)$ is uppersemicontinuous in there; see [36, cor. 5.1].

Next, we recall from [36, lemma 4.2] a simple geometric fact: if $x \in I_{r_{0}}(\partial W)$, and $\rho>0$ is such that $\operatorname{dist}(x, \partial W) \leq \rho$ and $B_{\rho}(x) \subset I_{r_{0}}(\partial W)$, then

$$
\tilde{B}_{\rho}(x) \subset B_{5 \rho}(x) \text {. }
$$

We are now in the position to prove (B.1). First of all we recall that, since $S$ defines a multiplicity 1 stationary varifold in $\Omega$, we have

$$
\mathcal{H}^{n}\left(S \cap B_{r}(x)\right) \geq \omega_{n} r^{n} \quad \forall x \in S, B_{r}(x) \Subset \Omega .
$$

In particular, B.1 holds with $c=\omega_{n}$ for all $x \in S \backslash I_{r_{0} / 6}(\partial W)$ as soon as $r<r_{0} / 6$. Therefore we can assume that

$$
x \in \operatorname{cl}(S) \cap I_{r_{0} / 6}(\partial W) .
$$

We first notice that we have $\sigma(x) \geq 1$ : by upper semicontinuity of $\sigma$ on $\operatorname{cl}(S) \cap$ $I_{r_{0} / 6}(\partial W)$, we just need to show this when, in addition to $B .9$, we have $x \in S$, and indeed in this case,

$$
\sigma(x) \geq \lim _{\rho \rightarrow 0^{+}} \frac{\mathcal{H}^{n}\left(S \cap B_{\rho}(x)\right)}{\omega_{n} \rho^{n}} \geq 1,
$$

thanks to $(\mathrm{B} .8)$; this proves $\sigma(x) \geq 1$. Now we fix $r<5 r_{0} / 6$ and distinguish two cases depending on the validity of

$$
\operatorname{dist}(x, \partial W)>\frac{r}{5}
$$

If $(\mathrm{B} .10)$ holds, then by (B.8),

$$
\mathcal{H}^{n}\left(S \cap B_{r}(x)\right) \geq \mathcal{H}^{n}\left(S \cap B_{r / 5}(x)\right) \geq \omega_{n}\left(\frac{r}{5}\right)^{n}
$$

thus proving (B.1). If $\operatorname{dist}(x, \partial W) \leq r / 5$, then, thanks to the obvious inclusion $B_{r}(x) \subset I_{r_{0}}(\partial W)$, we can apply (B.7) with $\rho=r / 5$ to find $\widetilde{B}_{r / 5}(x) \subset B_{r}(x)$. In this way, by exploiting $\sigma(x) \geq 1$ and (B.4), we get

$$
\begin{aligned}
c(n) r^{n} & \leq \sigma(x) \omega_{n}\left(\frac{r}{5}\right)^{n} \\
& \leq\left(\mathcal{H}^{n}\left(S \cap B_{r / 5}(x)\right)+\mathcal{H}^{n}\left(S \cap \widetilde{B}_{r / 5}(x)\right)\right) e^{C r / 5} \\
& \leq 2 \mathcal{H}^{n}\left(S \cap B_{r}(x)\right) e^{C r_{0}} \leq 4 \mathcal{H}^{n}\left(S \cap B_{r}(x)\right)
\end{aligned}
$$


up to further decreasing $r_{0}$.

\section{Appendix C A Classical Variational Argument}

Let $(K, E)$ be a generalized minimizer of $\psi(\varepsilon)$. In Theorem 1.6 , we have proved that if $f: \Omega \rightarrow \Omega$ is a diffeomorphism such that $|f(E)|=|E|$, then

$$
\mathcal{F}(K, E) \leq \mathcal{F}(f(K), f(E)) .
$$

Here we show how to deduce from (C.1) the existence of $\lambda \in \mathbb{R}$ such that

$$
\lambda \int_{\partial^{*} E} X \cdot v_{E} d \mathcal{H}^{n}=\int_{\partial^{*} E} \operatorname{div}^{K} X d \mathcal{H}^{n}+2 \int_{K \backslash \partial^{*} E} \operatorname{div}^{K} X d \mathcal{H}^{n}
$$

for every $X \in C_{c}^{1}\left(\mathbb{R}^{n+1} ; \mathbb{R}^{n+1}\right)$ with $X \cdot v_{\Omega}=0$ on $\partial \Omega$. This is proved following a classical argument; see, e.g., [42, theorem 17.20].

We first treat the case when we also have

$$
\int_{\partial^{*} E} X \cdot v_{E} d \mathcal{H}^{n}=0
$$

In this case, let $Y \in C_{c}^{1}\left(\Omega ; \mathbb{R}^{n+1}\right)$ be such that

$$
\int_{\partial^{*} E} Y \cdot v_{E} d \mathcal{H}^{n}=1
$$

and set

$$
f_{t, s}(x)=x+t X(x)+s Y(x) \quad x \in \Omega .
$$

Given that $X \cdot v_{\Omega}=0$ on $\partial \Omega$ and that $\partial \Omega$ is smooth, it is easily seen that for $t$ and $s$ sufficiently small, $f_{t, s}$ is a diffeomorphism from $\Omega$ to $\Omega$. In particular, the map

$$
\varphi(t, s)=\left|f_{t, s}(E)\right|
$$

is such that $\varphi(0,0)=|E|,(\partial \varphi / \partial t)(0,0)=0$ by (C.3), and $(\partial \varphi / \partial s)(0,0)=1$ by the assumption on $Y$, so that, by the implicit function theorem we have $\varphi(t, s(t))=$ $|E|$ for every $t$ sufficiently small and for $s(t)=\mathrm{O}\left(t^{2}\right)$. Setting $g_{t}=f_{t, s(t)}$, by (C.1), we find that

$$
m(t)=2 \mathcal{H}^{n}\left(g_{t}(K) \backslash \partial^{*} g_{t}(E)\right)+\mathcal{H}^{n}\left(\Omega \cap \partial^{*} g_{t}(E)\right)
$$

has a minimum at $t=0$. By Lemma A.1, we can write

$$
m(t)=2 \mathcal{H}^{n}\left(g_{t}\left(K \backslash \partial^{*} E\right)\right)+\mathcal{H}^{n}\left(g_{t}\left(\Omega \cap \partial^{*} E\right)\right) .
$$

By the area formula, and since $s(t)=\mathrm{O}\left(t^{2}\right)$ gives $\left.\left(\partial g_{t} / \partial t\right)\right|_{t=0}=X$, we deduce the validity of (C.2) when (C.3) holds. Let us now consider two fields $X_{k} \in$ $C_{c}^{1}\left(\mathbb{R}^{n+1} ; \mathbb{R}^{n+1}\right), k=1,2$, with $X_{k} \cdot v_{\Omega}=0$ on $\partial \Omega$ and set

$$
X=X_{1}-\frac{\int_{\partial^{*} E} X_{1} \cdot v_{E} d \mathcal{H}^{n}}{\int_{\partial^{*} E} X_{2} \cdot v_{E} d \mathcal{H}^{n}} X_{2}
$$


In this way $X$ satisfies $(\mathrm{C} .3)$, and thus $\overline{\mathrm{C} .2}$; as a consequence, the quantity

$$
\frac{\int_{\partial^{*} E} \operatorname{div}^{K} X_{k} d \mathcal{H}^{n}+2 \int_{K \backslash \partial^{*} E} \operatorname{div}^{K} X_{k} d \mathcal{H}^{n}}{\int_{\partial^{*} E} X_{k} \cdot v_{E} d \mathcal{H}^{n}}
$$

has the same value for $k=1,2$.

Acknowledgment. We thank an anonymous referee for several useful remarks that helped us improve the quality of the paper. Antonello Scardicchio has contributed to many inspiring discussions on the physical background of this work. This work was completed during Spring 2019 while FM was first a member of IAS in Princeton, through support from the Charles Simonyi Endowment, and then a visitor of ICTP in Trieste. All the authors were supported by the NSF grants DMS-1565354, DMS-RTG-1840314, and DMS-FRG-1854344.

\section{Bibliography}

[1] Almgren, F. J., Jr. Existence and regularity almost everywhere of solutions to elliptic variational problems with constraints. Mem. Amer. Math. Soc. 4 (1976), no. 165, viii+199 pp. doi:10.1090/memo/0165

[2] Ambrosio, L.; Colesanti, A.; Villa, E. Outer Minkowski content for some classes of closed sets. Math. Ann. 342 (2008), no. 4, 727-748. doi:10.1007/s00208-008-0254-z

[3] Ambrosio, L.; Fusco, N.; Pallara, D. Functions of bounded variation and free discontinuity problems, Oxford Mathematical Monographs. The Clarendon Press, Oxford University Press, New York, 2000.

[4] Bayle, V.; Rosales, C. Some isoperimetric comparison theorems for convex bodies in Riemannian manifolds. Indiana Univ. Math. J. 54 (2005), no. 5, 1371-1394. doi:10.1512/iumj.2005.54.2575

[5] Brezis, H.; Coron, J.-M. Multiple solutions of $H$-systems and Rellich's conjecture. Comm. Pure Appl. Math. 37 (1984), no. 2, 149-187. doi:10.1002/cpa.3160370202

[6] Caffarelli, L.; De Silva, D.; Savin, O. Obstacle-type problems for minimal surfaces. Comm. Partial Differential Equations 41 (2016), no. 8, 1303-1323.

[7] Caffarelli, L.; De Silva, D.; Savin, O. The two membranes problem for different operators. Ann. Inst. H. Poincaré Anal. Non Linéaire 34 (2017), no. 4, 899-932. doi:10.1016/j.anihpc.2016.05.006

[8] Caffarelli, L. A.; Jerison, D.; Kenig, C. E. Some new monotonicity theorems with applications to free boundary problems. Ann. of Math. (2) 155 (2002), no. 2, 369-404. doi:10.2307/3062121

[9] Colding, T. H.; Minicozzi, W. P., II. A course in minimal surfaces. Graduate Studies in Mathematics, 121. American Mathematical Society, Providence, R.I., 2011. doi: $10.1090 / \mathrm{gsm} / 121$

[10] David, G. Should we solve Plateau's problem again? Advances in analysis: the legacy of Elias M. Stein, 108-145. Princeton Mathematical Series, 50. Princeton University Press, Princeton, N.J., 2014.

[11] De Lellis, C. Rectifiable sets, densities and tangent measures. Zurich Lectures in Advanced Mathematics. European Mathematical Society (EMS), Zürich, 2008. doi:10.4171/044

[12] De Lellis, C.; De Rosa, A.; Ghiraldin, F. A direct approach to the anisotropic Plateau problem. Adv. Calc. Var. 12 (2019), no. 2, 211-223. doi:10.1515/acv-2016-0057

[13] De Lellis, C.; Ghiraldin, F.; Maggi, F. A direct approach to Plateau's problem. J. Eur. Math. Soc. (JEMS) 19 (2017), no. 8, 2219-2240. doi:10.4171/JEMS/716

[14] De Pauw, T.; Hardt, R. Size minimization and approximating problems. Calc. Var. Partial Differential Equations 17 (2003), no. 4, 405-442. doi:10.1007/s00526-002-0177-6 
[15] De Philippis, G.; De Rosa, A.; Ghiraldin, F. A direct approach to Plateau's problem in any codimension. Adv. Math. 288 (2016), 59-80. doi:10.1016/j.aim.2015.10.007

[16] De Philippis, G.; Maggi, F. Regularity of free boundaries in anisotropic capillarity problems and the validity of Young's law. Arch. Ration. Mech. Anal. 216 (2015), no. 2, 473-568. doi:10.1007/s00205-014-0813-2

[17] De Philippis, G.; Maggi, F. Dimensional estimates for singular sets in geometric variational problems with free boundaries. J. Reine Angew. Math. 725 (2017), 217-234. doi:10.1515/crelle2014-0100

[18] De Rosa, A. Minimization of anisotropic energies in classes of rectifiable varifolds. SIAM J. Math. Anal. 50 (2018), no. 1, 162-181. doi:10.1137/17M1112479

[19] del Pino, M.; Kowalczyk, M.; Wei, J. The Toda system and clustering interfaces in the AllenCahn equation. Arch. Ration. Mech. Anal. 190 (2008), no. 1, 141-187. doi:10.1007/s00205008-0143-3

[20] Dugundji, J. Topology. Allyn and Bacon, Boston, Mass., 1966.

[21] Duzaar, F.; Fuchs, M. On the existence of integral currents with prescribed mean curvature vector. Manuscripta Math. 67 (1990), no. 1, 41-67. doi:10.1007/BF02568422

[22] Fall, M. M. Area-minimizing regions with small volume in Riemannian manifolds with boundary. Pacific J. Math. 244 (2010), no. 2, 235-260. doi:10.2140/pjm.2010.244.235

[23] Fang, Y. Existence of minimizers for the Reifenberg plateau problem. Ann. Sc. Norm. Super. Pisa Cl. Sci. (5) 16 (2016), no. 3, 817-844.

[24] Fang, Y.; Kolasiński, S. Existence of solutions to a general geometric elliptic variational problem. Calc. Var. Partial Differential Equations 57 (2018), no. 3, Paper No. 91, 71 pp. doi:10.1007/s00526-018-1348-4

[25] Federer, H. Geometric measure theory. Die Grundlehren der mathematischen Wissenschaften, 153. Springer, New York, 1969.

[26] Finn, R. Equilibrium capillary surfaces. Die Grundlehren der mathematischen Wissenschaften, 284. Springer, New York, 1986. doi:10.1007/978-1-4613-8584-4

[27] Giaquinta, M.; Martinazzi, L. An introduction to the regularity theory for elliptic systems, harmonic maps and minimal graphs. Appunti. Scuola Normale Superiore di Pisa (Nuova Serie), 2. Edizioni della Normale, Pisa, 2005.

[28] Giusteri, G. G.; Lussardi, L.; Fried, E. Solution of the Kirchhoff-Plateau problem. J. Nonlinear Sci. 27 (2017), no. 3, 1043-1063. doi:10.1007/s00332-017-9359-4

[29] Giusti, E. On the equation of surfaces of prescribed mean curvature. Existence and uniqueness without boundary conditions. Invent. Math. 46 (1978), no. 2, 111-137. doi:10.1007/BF01393250

[30] Grüter, M.; Jost, J. Allard type regularity results for varifolds with free boundaries. Ann. Scuola Norm. Sup. Pisa Cl. Sci. (4) 13 (1986), no. 1, 129-169.

[31] Harrison, J. Soap film solutions to Plateau's problem. J. Geom. Anal. 24 (2014), no. 1, 271-297. doi:10.1007/s12220-012-9337-x

[32] Harrison, J.; Pugh, H. Existence and soap film regularity of solutions to Plateau's problem. Adv. Calc. Var. 9 (2016), no. 4, 357-394. doi:10.1515/acv-2015-0023

[33] Harrison, J.; Pugh, H. Plateau's problem. Open problems in mathematics, 273-302. Springer, Cham, 2016.

[34] Harrison, J.; Pugh, H. Solutions to the Reifenberg Plateau problem with cohomological spanning conditions. Calc. Var. Partial Differential Equations 55 (2016), no. 4, Art. 87, 37. doi:10.1007/s00526-016-1022-7

[35] Harrison, J.; Pugh, H. General methods of elliptic minimization. Calc. Var. Partial Differential Equations 56 (2017), no. 4, Art. 123, 25.

[36] Kagaya, T.; Tonegawa, Y. A fixed contact angle condition for varifolds. Hiroshima Math. J. 47 (2017), no. 2, 139-153. 
[37] King, D.; Maggi, F.; Stuvard, S. Collapsing and the convex hull property in a soap film capillarity model. Ann. Inst. H. Poincaré Anal. Non Linéaire, ISSN 0294-1449. doi:10.1016/j.anihpc.2021.02.005

[38] King, D.; Maggi, F.; Stuvard, S. Smoothness of collapsed regions in a capillarity model for soap films. Preprint, 2020. arXiv:2007.14868 [math.AP]

[39] King, D.; Maggi, F.; Stuvard, S. Plateau's problem as a singular limit of capillarity problems. Comm. Pure Appl. Math. Preprint, 2021. doi:10.1002/cpa.21951

[40] Leoni, G.; Murray, R. Second-order $\Gamma$-limit for the Cahn-Hilliard functional. Arch. Ration. Mech. Anal. 219 (2016), no. 3, 1383-1451. doi:10.1007/s00205-015-0924-4

[41] Leoni, G.; Murray, R. A note regarding second-order $\Gamma$-limits for the Cahn-Hilliard functional. Preprint, 2017. arXiv:1705.00606 [math.AP]

[42] Maggi, F. Sets of finite perimeter and geometric variational problems. An introduction to geometric measure theory. Cambridge Studies in Advanced Mathematics, 135. Cambridge University Press, Cambridge, 2012. doi:10.1017/CBO9781139108133

[43] Maggi, F.; Mihaila, C. On the shape of capillarity droplets in a container. Calc. Var. Partial Differential Equations 55 (2016), no. 5, Art. 122, 42 pp. doi:10.1007/s00526-016-1056-x

[44] Maggi, F.; Stuvard, S.; Scardicchio, A. Soap films with gravity and almost-minimal surfaces. Discrete Contin. Dyn. Syst. 39 (2019), no. 12, 6877-6912. doi:10.3934/dcds.2019236

[45] Mattila, P. Geometry of sets and measures in Euclidean spaces. Fractals and rectifiability. Cambridge Studies in Advanced Mathematics, 44. Cambridge University Press, Cambridge, 1995. doi:10.1017/CBO9780511623813

[46] Preiss, D. Geometry of measures in $\mathbf{R}^{n}$ : distribution, rectifiability, and densities. Ann. of Math. (2) 125 (1987), no. 3, 537-643. doi:10.2307/1971410

[47] Simon, L. Lectures on geometric measure theory. Proceedings of the Centre for Mathematical Analysis, Australian National University, 3. Australian National University, Centre for Mathematical Analysis, Canberra, 1983.

[48] Taylor, J. E. The structure of singularities in soap-bubble-like and soap-film-like minimal surfaces. Ann. of Math. (2) 103 (1976), no. 3, 489-539. doi:10.2307/1970949

[49] Villa, E. On the outer Minkowski content of sets. Ann. Mat. Pura Appl. (4) 188 (2009), no. 4, 619-630. doi:10.1007/s10231-008-0093-2

[50] White, B. Currents and flat chains associated to varifolds, with an application to mean curvature flow. Duke Math. J. 148 (2009), no. 1, 41-62. doi:10.1215/00127094-2009-019 
DARREN KING

Department of Mathematics

The University of Texas at Austin

2515 Speedway, Stop C1200

Austin, TX 78712-1202

USA

E-mail: king@math.utexas.edu

SALVATORE STUVARD

Department of Mathematics

The University of Texas at Austin

2515 Speedway, Stop C1200

Austin, TX 78712-1202

USA

E-mail: stuvarde

math.utexas.edu

Received November 2019.
FRANCESCO MAGGI

Department of Mathematics

The University of Texas at Austin

2515 Speedway, Stop C1200

Austin, TX 78712-1202

USA

E-mail:maggi@

math.utexas.edu 